\title{
AmFm and lithium gap stars
}

\section{Stellar evolution models with mass loss}

\author{
M. Vick ${ }^{1,3}$, G. Michaud ${ }^{2,3}$, J. Richer ${ }^{3}$, and O. Richard ${ }^{1}$ \\ 1 GRAAL UMR 5024, Université Montpellier II, CC072, Place E. Bataillon, 34095 Montpellier Cedex 05, France \\ 2 LUTH, Observatoire de Paris, CNRS, Université Paris Diderot, 5 place Jules Janssen, 92190 Meudon, France \\ 3 Département de physique, Université de Montréal, Montréal, Québec, H3C 3J7, Canada \\ e-mail: [mathieu.vick; jacques.richer]@umontreal.ca; michaudg@astro.umontreal.ca; \\ olivier.richard@graal.univ-montp2.fr \\ Received 23 February 2010 / Accepted 13 June 2010
}

\section{ABSTRACT}

\begin{abstract}
Aims. A thorough study of the effects of mass loss on internal and surface abundances of A and F stars is carried out in order to constrain mass loss rates for these stars, as well as further elucidate some of the processes which compete with atomic diffusion. Methods. Self-consistent stellar evolution models of 1.3 to $2.5 M_{\odot}$ stars including atomic diffusion and radiative accelerations for all species within the OPAL opacity database were computed with mass loss and compared to observations as well as previous calculations with turbulent mixing.

Results. Models with unseparated mass loss rates between $5 \times 10^{-14}$ and $10^{-13} M_{\odot} \mathrm{yr}^{-1}$ reproduce observations for many cluster AmFm stars as well as Sirius A and $o$ Leonis. These models also explain cool Fm stars, but not the Hyades lithium gap. Like turbulent mixing, these mass loss rates reduce surface abundance anomalies; however, their effects are very different with respect to internal abundances. For most of the main-sequence lifetime of an A or F star, surface abundances in the presence of such mass loss depend on separation which takes place between $\log \Delta M / M_{*}=-6$ and -5 .

Conclusions. The current observational constraints do not allow us to conclude that mass loss is to be preferred over turbulent mixing (induced by rotation or otherwise) in order to explain the AmFm phenomenon. Internal concentration variations which could be detectable through asteroseismic tests should provide further information. If atomic diffusion coupled with mass loss are to explain the Hyades Li gap, the wind would need to be separated.
\end{abstract}

Key words. diffusion - stars: mass-loss - stars: evolution - stars: chemically peculiar - stars: abundances open clusters and associations: individual: Hyades

\section{Astrophysical context}

As instruments become more sophisticated and precise observations are readily made available for a growing number of chemical elements, additional constraints are steering the evolution of stellar models. The inclusion of atomic diffusion and radiative accelerations into the standard stellar evolution model resulted in some early success in describing abundance patterns for AmFm stars (Turcotte et al. 1998a). In these models, particle transport within the radiative zone was calculated from all physics known through first principles. However, the calculated surface anomalies were greater than the observed anomalies, and it was determined that additional transport processes were flattening the surface abundance patterns. With the addition of turbulent mixing, the Montreal group's stellar evolution code (Richer et al. 2000; Richard et al. 2001; Michaud et al. 2004, 2005; Talon et al. 2006) was able to explain and reproduce the particular abundances of many cluster and field stars with a single tunable parameter: the mixed mass. Nonetheless, other studies have suggested that mass loss could also reduce the predicted anomalies to observed levels (Michaud et al. 1983; Michaud \& Charland 1986; Alecian 1996). Since these studies considered static stellar models incorporating a limited number of species, a rigorous investigation of the effects of mass loss on stellar chemical evolution is warranted. Previously, Vauclair \& Charbonnel (1995) have introduced mass loss to reduce the effect of Li settling in Pop II evolutionary models.
AmFm stars $\left(7000 \leq T_{\text {eff }} \leq 10000 \mathrm{~K}\right)$ are slowly rotating $\left(v_{\text {rot }}<100 \mathrm{~km} \mathrm{~s}^{-1}\right.$, Abt 2000), non-magnetic stars of the mainsequence (MS). They are interesting candidates for testing evolutionary models because they lie within the temperature range for which the depth of the surface convection zone varies quite rapidly. Chemical separation within the stable radiative zones of these stars generates surface abundance anomalies within timescales that depend strongly on the depth of the surface convection zone, although the exact depth at which the separation occurs is still debated. The original explanation for these chemically peculiar stars stipulated that the separation occurred immediately below the surface H convection zone (Watson 1971; Alecian 1986, 1996); however, with the inclusion of turbulent mixing, more recent models (Richer et al. 2000) have suggested that separation occurs much deeper in the star $(T>200000 \mathrm{~K})$. Though the second scenario has had success not only with AmFm stars, but also with Pop II (Richard et al. 2005; Korn et al. 2006) and Horizontal Branch (HB) stars (Michaud et al. 2007, 2008), it is still premature to accept turbulence as the soughtafter macrospcopic process since all models with turbulence necessarily involve at least one adjustable parameter. Turbulence models often implicitely assume a link between turbulence and rotation; however, even the most slowly rotating AmFm stars have anomalies which are significantly smaller than those obtained with atomic diffusion only models. This suggests the presence of a competing process even in non rotating stars. 
Typical anomalies on the surface of AmFm stars include overabundances of iron peak elements, as well as underabundances of $\mathrm{Ca}$ and/or Sc (see Cayrel et al. 1991, for a more complete description). Recent studies have obtained abundance determinations for numerous $\mathrm{A}$ and $\mathrm{F}$ stars of open clusters for a number of chemical species (Burkhart \& Coupry 2000; Hui-Bon-Hoa 2000; Monier 2005; Fossati et al. 2007; Gebran et al. 2008; Gebran \& Monier 2008) in an effort to confront relatively well constrained stars to current evolutionary models. The advantage of observing cluster stars is that they generally have the same age and initial metallicity, which greatly facilitates a comparison with models.

At the cool end of the Fm star domain, the well documented $\mathrm{Li}$ dip, first observed in the Hyades open cluster (Boesgaard \& Tripicco 1986), has challenged theoretical astronomers for decades (Michaud 1986; Vauclair 1988; Michaud \& Charbonneau 1991; Talon \& Charbonnel 2005). The lithium abundance has also been observed in many other open clusters (e.g. Balachandran 1995; Burkhart \& Coupry 2000; AnthonyTwarog et al. 2009). The Be abundances in these stars (Boesgaard \& King 2002; Boesgaard et al. 2004; Randich et al. 2007) provide additional constraints on particle transport. Recently, Talon \& Charbonnel $(2003,2005)$ have modeled shear turbulence induced by differential rotation and mixing induced by internal gravity waves in order to describe both the hot and cold side of the dip. Other models have also explored the effects of horizontal $\mu$-gradients on rotationally induced mixing in these stars (Palacios et al. 2003). However, the potential effects of atomic diffusion, more specifically of radiative accelerations, in competition with mass loss, were not fully investigated.

At some level, mass loss is present in all stars, and it is important to quantify its effects on observed abundances. Unfortunately, for A and $\mathrm{F}$ stars in particular, the mass loss rates are not known. In $\mathrm{O}$ and $\mathrm{B}$ stars, the radiatively driven winds produce mass loss rates as important as $10^{-4} M_{\odot} \mathrm{yr}^{-1}$ (Lamers \& Cassinelli 1999). In colder stars of types $G$ and $K$, winds are driven by active coronas. The best known example is the solar wind which has a mass loss rate of $2 \times 10^{-14} M_{\odot} \mathrm{yr}^{-1}$ (Feldman et al. 1977). For intermediate stars, our understanding is at best nebulous. Abbott (1982) suggests that radiative accelerations are too small in stars with $T_{\text {eff }} \leq 10000 \mathrm{~K}$ for radiatively driven mass loss to be significant. On the other hand, the thinning of the surface convection zone in $\mathrm{F}$ and particularly in A stars might be too important for solar type winds to exist (Parker 1960). Are winds of $\mathrm{A}$ and $\mathrm{F}$ stars driven by radiation, coronal heating, both or neither? What are the expected mass loss rates? Both of these questions remain unanswered, and answering one could shed light on the other.

Observational constraints on $\mathrm{A}$ and $\mathrm{F}$ star mass loss rates are limited. Lanz \& Catala (1992) as well as Brown et al. (1990) gave an upper limit of $10^{-10} M_{\odot} \mathrm{yr}^{-1}$ for main sequence A stars. Asymmetries in Mg II spectral lines of Sirius A led Bertin et al. (1995) to conclude that mass loss is present with a rate between $10^{-13} M_{\odot} \mathrm{yr}^{-1}$ and $10^{-12} M_{\odot} \mathrm{yr}^{-1}$. On the theoretical side, the radiatively driven wind model of Babel (1995) suggests a mass loss rate of $10^{-16} M_{\odot} \mathrm{yr}^{-1}$ for A stars. However, according to his results, only heavier elements are evacuated by the radiative field. Similarly, Michaud et al. (1983, see also Michaud \& Charland 1986) suggested that mass loss rates between $10^{-14} M_{\odot} \mathrm{yr}^{-1}$ and $10^{-15} M_{\odot} \mathrm{yr}^{-1}$ could satisfy observational constraints from $\mathrm{CP}$ star surface anomalies. Given the large disparity in values, the mass loss rates used in this study will be constrained strictly by surface abundance variations (see also Sect. 4.2).

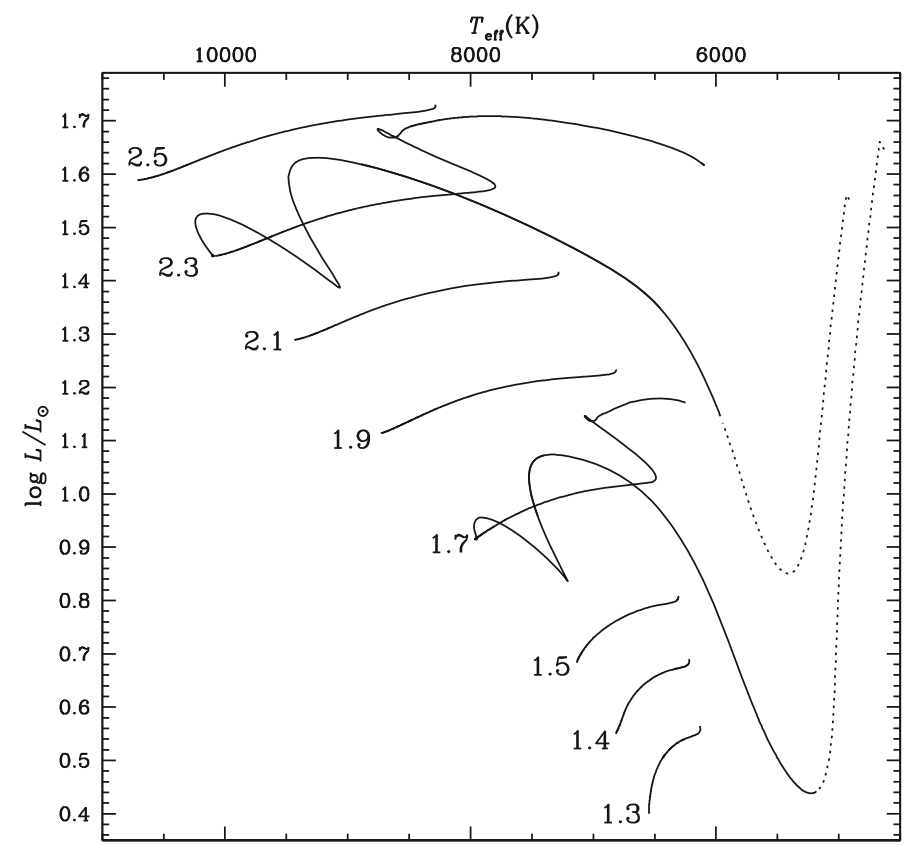

Fig. 1. H-R diagram for all the models shown in Fig. 4. Though all models were calculated from the PMS to the bottom of the subgiant branch, the complete tracks are only shown for the 1.7 and $2.3 M_{\odot}$ models. All other models are shown only on the main-sequence, which, for the purpose of this plot, is considered to span from the time at which $1 \%$ of core $\mathrm{H}$ is burned to the time at which $95 \%$ is burned. The dotted line represents evolution on the PMS until diffusion starts (i.e. until a radiative zone appears).

In this paper, we consider mass loss in non rotating stars. In these stars, mass loss is arguably the only macroscopic process competing with atomic diffusion within the radiative zones. We will start with a brief description of our stellar evolution code in Sect. 2 after which we will discuss the method for calculating radiative accelerations for lithium, beryllium and boron (Sect. 3). In Sect. 4 we will describe the treatment of mass loss. In Sect. 5 we will discuss its effect on internal structure and surface abundances as the models move along the main-sequence and the subgiant branch. In Sects. 6 and 7 we will compare our models to turbulence models and observations respectively. In Sect. 8, a brief overview of the main results will be followed by a discussion on how asteroseismology could help decipher the effects of advective processes including meridional circulation from those incurred through turbulent processes caused by differential rotation.

\section{Calculations}

This paper is part of the Montreal stellar model development project (Richard et al. 2001; Turcotte et al. 1998b and references therein). The models were evolved from the initially chemically homogeneous pre-main-sequence (see Fig. 1) with the solar abundance mix listed in Table 1 of Turcotte et al. (1998b). The transport of chemical elements in 1D (one dimension) is solved within the basic framework established by Burgers (1969). The chemical transport equation considers all effects of radiative accelerations and atomic diffusion, and it is solved for $28 \mathrm{chem}$ ical elements and isotopes at every mesh point (the number of points varies from 800 to about 2800) for each time step. Atomic diffusion is allowed to operate as soon as a radiative zone appears. The radiative acelerations $\left(g_{\mathrm{rad}}\right)$ and the Rosseland mean 
opacity are continuously updated; the treatment of chemical transport is thereby fully self-consistent. The atomic diffusion coefficients were taken from Paquette et al. (1986, see also Michaud \& Proffitt 1993). The Krishna Swamy $T-\tau$ relation (Krishna-Swamy 1966) was imposed as the boundary condition in the atmosphere (this choice was motivated by results presented in VandenBerg et al. 2008). Semiconvection was included as described in Richard et al. (2001), following Kato (1966), Langer et al. (1985), and Maeder (1997).

The chosen values of the mixing length parameter and initial He fraction are respectively $\alpha=2.096$ and $Y_{0}=0.27769$ (see Model H of Turcotte et al. 1998b), which are calibrated by fitting the current solar radius and luminosity. We chose $Z_{0}=0.01999$ as the initial mass fraction of metals ${ }^{1}$. Some models were also calculated for $Z_{0}=0.01$ and $Z_{0}=0.03$.

These are the first fully self-consistent stellar models which include mass loss. Models were calculated from $1.30 M_{\odot}$ to $2.50 M_{\odot}$. The mass loss rates considered vary from $1 \times 10^{-16} M_{\odot} \mathrm{yr}^{-1}$ to $1 \times 10^{-12} M_{\odot} \mathrm{yr}^{-1}$. Our treatment of mass loss will be further discussed in Sect. 4.1.

\section{Radiative accelerations}

Rosseland opacities as well as radiative accelerations are continuously computed for all 28 species as the relative concentration of each species varies with time. For all elements included in the OPAL database (Iglesias \& Rogers 1996), the radiative acceleration calculations are carried out using direct summations over the actual spectrum (i.e. opacity sampling, see Richer et al. 1998). At large optical depths (where the diffusion approximation is valid), the radiative acceleration, $g_{\mathrm{rad}}(A)$, of an element $A$ at a radius $r$ in a star may be approximated by:

$g_{\mathrm{rad}}(A)=\frac{1}{4 \pi r^{2}} \frac{L_{r}^{\mathrm{rad}}}{c} \frac{\kappa_{R}}{X_{A}} \int_{0}^{\infty} \frac{\kappa_{u}(A)}{\kappa_{u}(\text { total })} \mathcal{P}(u) \mathrm{d} u$,

where $\mathcal{P}(u)$, the normalized black body flux distribution, is given by:

$\mathcal{P}(u) \equiv \frac{15}{4 \pi^{4}} \frac{u^{4} e^{u}}{\left(e^{u}-1\right)^{2}}$.

The radiative luminosity at a radius $r$ is $L_{r}^{\mathrm{rad}}, X_{A}$ is the mass fraction of $A, \kappa_{\mathrm{R}}$ is the Rosseland opacity, $\kappa_{u}$ (total) and $\kappa_{u}(A)$ are respectively the total opacity and the contribution of $A$ to the total opacity at the frequency $u$ defined by:

$u \equiv h v / k T$.

Since the competition for photons between element $A$ and all other elements present in the plasma determines the value of $g_{\mathrm{rad}}(A)$, atomic data is required for all species in order to compute $g_{\text {rad }}$ for any given element.

The corrections for redistribution of momentum are from Gonzalez et al. (1995) and LeBlanc et al. (2000).

\footnotetext{
1 Asplund et al. $(2005,2009)$ have proposed a downward revision of the solar abundances of some metals; however we have chosen to keep the previous abundances until their determinations are reconciled with helioseismology (Delahaye \& Pinsonneault 2006; Basu et al. 2007 and references therein). The abundance of $\mathrm{O}$, the third most abundant element, is particularly uncertain (Caffau et al. 2008; Delahaye et al. 2010).
}

\subsection{Radiative accelerations for $\mathrm{Li}, \mathrm{Be}$ and $\mathrm{B}$}

\subsubsection{Methods}

$\mathrm{Li}, \mathrm{Be}$, and $\mathrm{B}$ are not included in OPAL since they are not significant in the calculation of Rosseland mean opacity ${ }^{2}$. We have nonetheless computed the radiative accelerations in a manner which is consistent with OPAL spectra. The various corrections determined by Richer et al. (1997) are also included. The atomic data required for $\mathrm{LiBeB}$ are taken from Wiese et al. (1966). Since these elements are not sufficiently abundant for pressure broadening to be important (the lines are never saturated), only oscillator strengths are required to compute opacities.

The calculation of $g_{\mathrm{rad}}$ for these elements is delicate because of two factors which can lead to large fluctuations: (1) non optimal frequency sampling and (2) random background changes. Both problems arise from the fact that $\mathrm{LiBeB}$ not only have very few lines contributing to their $g_{\mathrm{rad}}$, but these lines are also very narrow.

(1) The fluctuations due to sampling not being sufficiently refined has an effect on $\mathrm{Li}$ as well as on the background (see Fig. 2). The Doppler width of a ${ }^{7} \mathrm{Li}$ line is given by:

$\frac{\Delta u}{u}=\left[\frac{2 k T}{M c^{2}}\right]^{0.5}=5.1 \times 10^{-5} T_{5}^{0.5}$

where $u$ is the adimensional energy difference between the upper and lower levels of the transition, $M$ is the mass of the element, $T$ is the local temperature and $T_{5}=T / 10^{5} \mathrm{~K}$. In this case, one typically has $u \simeq 5$ around the most important temperature range, $\log T \simeq 5.3$, so that

$\Delta u_{\text {Doppler }}=\Delta u_{D} \simeq 7 \times 10^{-5}$

which is to be compared to the frequency grid interval, $\Delta u_{G}=$ $20 / 10^{4}=2 \times 10^{-3}$, of the OPAL spectra. Using opacity sampling for Li would imply that the line center would sometimes occur within $\Delta u_{D}$ of a grid point and sometimes up to $15 \Delta u_{D}$ away. If the sampling point occurs in the far tail of an important $\mathrm{Li}$ line, the $g_{\mathrm{rad}}(\mathrm{Li})$ value would be much smaller than if it occurs in its center.

Likewise, the sampling is not refined enough to reproduce all features of the background spectra (see Fig. 3 of Richer et al. 1998). Variations can therefore result from overlooking an important background line which occurs near a lithium line.

(2) The frequency sampling grid is a function of $u$ and not of $v$. Therefore, when one considers an adjacent grid point of different $T$, the background, as a function of $u$, changes, and the consequent random variation in background affects the flux available for a specific lithium line. This is largely due to the narrowness of most lines. For example, in the inset of Fig. 2, the Li line does not encounter any important Fe line for that specific $(\rho, T)$ table; however, in the inset of Fig. 1 of Richer \& Michaud (2005), which shows the same $u$ interval for a different $(\rho, T)$ table, the Li line is overlapped by a strong Fe line, and the available flux is consequently reduced.

One can alleviate these problems by combining a modified version of the opacity sampling method with the known position

\footnotetext{
${ }^{2}$ In Turcotte et al. (1998b) and Turcotte et al. (1998a), $g_{\text {rad }}(\mathrm{Li})$ and $g_{\mathrm{rad}}(\mathrm{Be})$ were approximated using formulae derived previously by Richer \& Michaud (1993). These are however less accurate than $g_{\mathrm{rad}}$ calculated using direct summations over the spectrum throughout stellar evolution (using Eq. (1)), which are described here and were used in Richer et al. (2000) and Richard et al. (2001).
} 


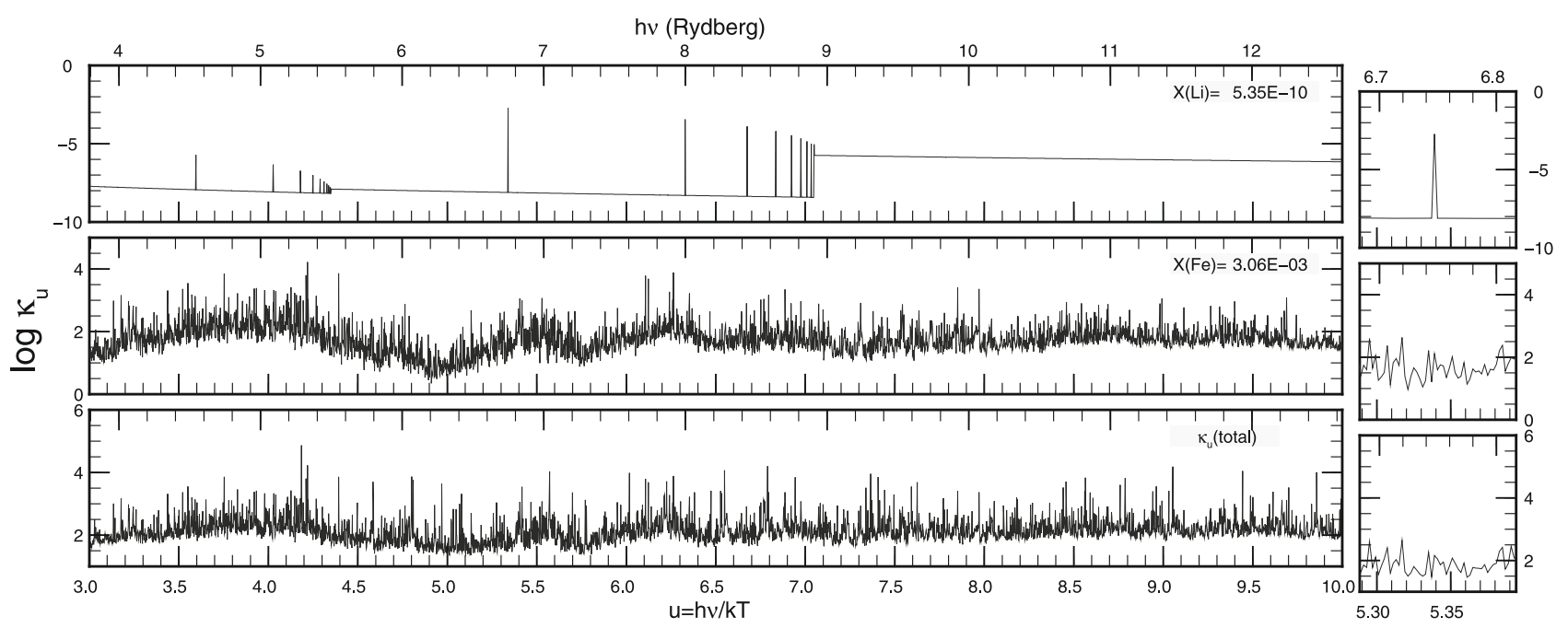

Fig. 2. Opacity spectra for $\mathrm{Li}, \mathrm{Fe}$ as well as the total opacity in $\mathrm{cm}^{2} \mathrm{~g}^{-1}$ at a depth where $\log T=5.3 \operatorname{and} \log \rho=-4.6$. The $u$ interval covers the range through which most of the flux passes. The spectrum is dominated by Fe lines. The inset on the right shows a zoom of the area which hosts the Li line which contributes the most to its $g_{\mathrm{rad}}$.

of all the line centers for $\mathrm{LiBeB}$. In order to reduce the fluctuations, while preparing the spectra of, say, Li, for the calculations of $g_{\mathrm{rad}}(\mathrm{Li})$, it was assumed that each Li line was spread uniformly over the $\Delta u_{G}$ interval in which the Li line center occurs (i.e. the Li lines become square functions of width $\Delta u_{G}$ and so it becomes impossible for the sampling to overlook them). This will leave fluctuations caused by random variations of the opacity background when the line center moves from one $\Delta u_{G}$ interval to another (2), but the variations due to non optimal sampling (1) will be reduced significantly.

Note that if the frequency sampling is refined (e.g. $10^{5}$ as in OP data) in order to better represent the background spectra, errors due to the inexact position (in frequency) of each background feature remain. The line center positions for Fe, the main contributor to the total opacity in this region, are only known to about $1 \%$.

The resulting error bars on $g_{\text {rad }}$ are discussed in Sect. 3 of Richer \& Michaud (2005). As an example, for Population I stars, there is a factor of 2 uncertainty for $g_{\mathrm{rad}}(\mathrm{Li})$. In Pop II stars, the uncertainty is much less important (e.g. only $3 \%$ for a star with $Z=0.0001)$.

The same errors and limitations should be expected for scandium around the minima of its $g_{\text {rad }}$ since the radiative accelerations in these regions are computed with only a few narrow lines. Unfortunately, Sc is not included in OPAL data, and its $g_{\text {rad }}$ must be calculated through alternate methods (LeBlanc \& Alecian 2008).

\subsubsection{Results}

The radiative accelerations for $\mathrm{Li}$ and Be are shown in Fig. 3 for a star of $1.55 M_{\odot}$ with a mass loss rate of $1 \times 10^{-13} M_{\odot} \mathrm{yr}^{-1}$ as well as for a star of $1.47 M_{\odot}$ with no mass loss. For both models, the radiative accelerations for $\mathrm{Be}$ are below gravity throughout the radiative zone, and throughout evolution. However, $g_{\mathrm{rad}}(\mathrm{Li})$ has a peak that approximately reaches gravity below the surface convection zone for the $1.55 M_{\odot}$ model. Up to $\log T \simeq 5.4, g_{\text {rad }}$ is seen to be almost solely temperature dependant as the curves for both models overlap.

In the $1.47 M_{\odot}$ model, $g_{\text {rad }}(\mathrm{Li})$ has about the same value as in the $1.55 M_{\odot}$ model for $\log T>5.4$, but, for $\log T<5.4$ it is

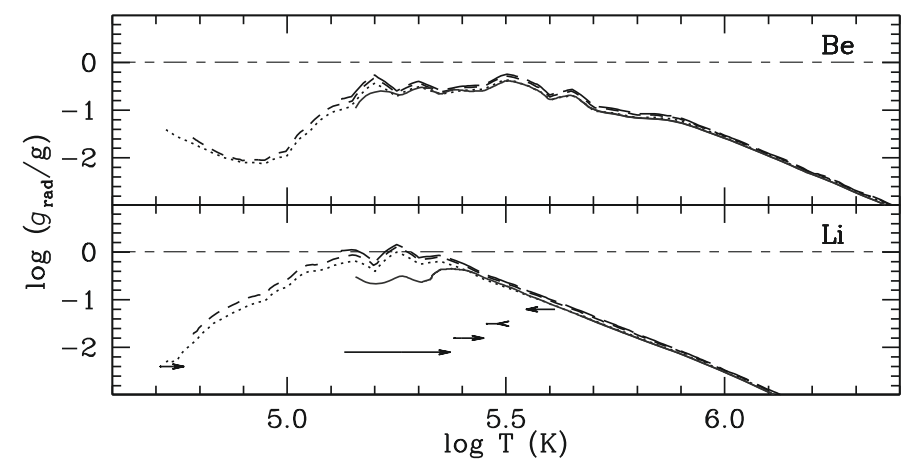

Fig. 3. Variation of radiative accelerations with temperature for $\mathrm{Li}$ and $\mathrm{Be}$ at $100 \mathrm{Myr}$ (dotted line), $700 \mathrm{Myr}$ (dashed line) and $1.3 \mathrm{Gyr}$ (long dashed line) in a $1.55 M_{\odot}$ model with a mass loss rate of $1 \times$ $10^{-13} M_{\odot} \mathrm{yr}^{-1}$ as well as at $100 \mathrm{Myr}$ (solid line) for a $1.47 M_{\odot}$ model with no mass loss. The curves extend to the bottom of the surface convection zone, on the left. Acceleration is normalized to local gravity. The horizontal arrows indicate the total movement of the bottom of the surface convection zone (BSCZ) for models of 1.40, 1.43, 1.45, 1.47 and $1.55 M_{\odot}$ from top to bottom (see text below).

reduced by the competition of $\mathrm{Fe}$, which absorbs most of the flux where the Li lines are important. This occurs when mass loss is small enough for Fe to accumulate in and above this region, as is the case for the $1.47 M_{\odot}$ model (or in the $1.50 M_{\odot}$ model with a mass loss rate of $10^{-14} M_{\odot} \mathrm{yr}^{-1}$ shown in Fig. 5). On the other hand, $g_{\mathrm{rad}}(\mathrm{Be})$ does not vary with $\mathrm{Fe}$ abundance likely because most Be lines, particularly the ones which contribute the most to its $g_{\text {rad }}$, lie outside of the frequency interval which is dominated by Fe lines.

The horizontal arrows in Fig. 3 show the total movement of the bottom of the surface convection zone (BSCZ) for 4 models without mass loss $\left(1.40,1.43,1.45\right.$ and $\left.1.47 M_{\odot}\right)$, as well as for a model of $1.55 M_{\odot}$ with a mass loss rate of $10^{-13} M_{\odot} \mathrm{yr}^{-1}$. The interval for which the BSCZ is plotted spans from $50 \mathrm{Myr}$ to $625 \mathrm{Myr}$ for all models except for the $1.47 M_{\odot}$, model which goes from $50 \mathrm{Myr}$ to $425 \mathrm{Myr}$ (the simulation's last converged model). For most models, the depth of the BSCZ does not vary much between 50 and $625 \mathrm{Myr}$ (most of the main-sequence lifetime before the age of the Hyades cluster); however, for the 
1.47 $M_{\odot}$ model, the BSCZ moves significantly and extends over an interval in which $g_{\text {rad }}(\mathrm{Li})$ also varies significantly.

If we compare the curves for the $1.55 M_{\odot}$ model with those shown in Fig. 1 of Richer \& Michaud (1993), we first note the similarity in the temperature dependence of the curves. For $\mathrm{Li}$, we have verified that they are nearly equal for $\log T>5.4$. The maxima occur at very nearly the same temperature as well. However, the maximum values of $g_{\mathrm{rad}}(\mathrm{Li})$ and $g_{\mathrm{rad}}(\mathrm{Be})$ are respectively about 2 and 6 times larger in Richer \& Michaud (1993). Furthermore, their results show very smooth curves compared to the many variations seen in our calculations.

Although there is a slight difference in stellar mass (a $1.54 M_{\odot}$ model is shown in Fig. 1 of Richer \& Michaud 1993), this cannot account for the relatively large differences close to the maximum. There are nonetheless a few other explanations. Our calculations were carried out with integrations over complete OPAL spectra for all species at each time step and each mesh point. Since these spectra were not available in 1993, the calculations of Richer \& Michaud (1993) were done using averaged spectra whose frequency dependence (Eq. (18) of Borsenberger et al. 1979) did not include the frequency dependence of Fe lines, particularly near $T \simeq 2 \times 10^{5} \mathrm{~K}$, where $g_{\mathrm{rad}}(\mathrm{Li})$ is affected the most. From Fig. 2, one can see that Fe lines dominate the region where $\mathrm{Li}$ lines are strongest. As Fe lines which occur near Li lines were absent in the old opacities, the available flux for Li lines is very different (see Sect. 3.1.1).

Moreover, the evolution of individual metal abundances and its impact on local opacity are not included in the calculations of Richer \& Michaud (1993). This is particularly important in AmFm stars since heavier metals such as iron and nickel tend to accumulate below the surface convection zone when mass loss is not too strong.

\section{Mass loss}

We first discuss how to include mass loss in evolutionary models (Sect. 4.1). In Sect. 4.2, we discuss a few theoretical analyses of coronal and radiatively driven winds followed by a brief look into separated winds ${ }^{3}$ (Sect. 4.3).

\subsection{Treatment of mass loss}

Mass loss is assumed spherical and chemically unseparated. If we simply apply mass conservation arguments, the net result of mass loss is the appearance of an outward flowing interior velocity due to the wind:

$v_{\mathrm{w}}(r)=-\frac{\dot{M}}{4 \pi r^{2} \rho} \frac{m_{r}}{M_{*}}$

where $\rho$ is the local density, $m_{r}$ is the mass interior to $r$ and $\dot{M}<0$. It should be pointed out that the $m_{r} / M_{*}$ factor in this equation has often been overlooked, but it will be shown to be a consequence of mass conservation (see Sect.4.1.1). Even for small mass loss rates, the $\rho^{-1}$ dependence leads to large velocities in the outer layers of the star. To avoid numerical instabilities due to the cancellation of two large quantities in the convective envelope (wind velocities and large turbulent velocities used to

\footnotetext{
3 In this paper, mass loss is separated when the abundances in the wind are not the same as in the photosphere. On the contratry, mass loss is unseparated when the abundances in the wind are the same as in the photosphere.
}

enforce convective mixing) and in order to have a Neumann surface boundary condition (no flux), mass loss was implemented as described in Charbonneau (1993). The main physical considerations are that the surface convection zone (SCZ) be fully mixed, and that the atmosphere be mixed with it by overshooting. Therefore, the ejected mass has the same composition as the SCZ. The mechanism by which mass is ejected from the star becomes irrelevant so long as the correct amount of mass is removed from the star. For each chemical species, the resulting transport equation is:

$$
\begin{aligned}
\rho \frac{\partial c}{\partial t}= & -\nabla \cdot\left[-\rho D \nabla \ln c+\rho\left(\boldsymbol{U}+\boldsymbol{U}_{w}\right) c\right] \\
& +\rho\left(S_{\mathrm{nuc}}+S_{w}\right) c
\end{aligned}
$$

with a Neumann condition imposed at the surface and with $\boldsymbol{U}_{w}$ and $S_{w}$ defined as:

$$
\begin{aligned}
& \boldsymbol{U}_{w}=\left\{\begin{array}{l}
v_{w} \hat{\boldsymbol{e}}_{\boldsymbol{r}} \text { under the SCZ, } \\
0 \text { in the SCZ; }
\end{array}\right. \\
& S_{w}=\left\{\begin{array}{l}
0 \text { under the SCZ, } \\
\frac{\dot{M}}{M_{\mathrm{CZ}}} \text { in the SCZ. }
\end{array}\right.
\end{aligned}
$$

Here, $c$ is the time and depth dependent composition, $D$ the total diffusion coefficient, $\boldsymbol{U}$ the advective part of the atomic diffusion velocity, $\boldsymbol{U}_{w}$ the interior velocity due to the wind, $M_{\mathrm{CZ}}$ the mass of the SCZ, $\dot{M}$ the mass loss rate, $S_{\text {nuc }}$ a source/destruction term linked to nuclear reactions and $S_{w}$ is a sink term linked to mass loss. The mass is simply removed from the surface convection zone which, given the assumed mixing, is equivalent to losing it through the stellar surface. The mass of the star is also continuously updated so that all quantities that depend on stellar mass are correctly calculated.

\subsubsection{Mass flux equation}

Mass loss has the effect of ejecting (or peeling) the outermost layers of a stellar model. The model must then be reconverged with a slightly reduced mass. This scenario can be described within the formalism of operator splitting. If a mass $\Delta M_{\mathrm{loss}}$ is removed in a given time step $\Delta t$, one needs to reconverge a model of mass $M_{1}=M_{0}-\Delta M_{\text {loss }}$ at time $t_{1}=t_{0}+\Delta t$ with the composition of the star kept unchanged as a function of $m_{r}$, the independent variable which is defined as the sphere of constant mass, $m_{r}$. Mass loss does not modify the abundance profiles with respect to $m_{r}$, although there is a change with respect to the stellar surface. For example, an abundance peak at the point $M_{0}-\Delta M_{\text {loss }}$ (or $\Delta M_{\text {loss }}$ below the surface before peeling), finds itself at $M_{1}$ (or at the surface) after the peeling but the mass interior to this point, $m_{r}$, has not changed.

To implement mass loss in a stellar evolution code, one can imagine the solution process to be broken down into 2 steps. In a first step, the model can be converged at a time $t_{1}$ as if there were no mass loss, with the composition changes due to nuclear reactions and diffusion processes. Then, the grid at $t_{1}$ is reinterpreted as corresponding to a mass $M_{1}=M_{0}-\Delta M_{\text {loss }}$ with all variables expressed as a function ${ }^{4}$ of $m_{r} / M_{*}$ except for the composition which is kept unchanged as a function of $m_{r}$. The star is then reconverged a second time at $t_{1}$ with a mass $M_{1}$ and with the concentration profiles which include the effect of mass loss. As more mass is lost, the model inches toward less massive structures, and so evolution progressively corresponds to that of a

${ }^{4}$ Here $M_{1}=M_{*}$ at $t_{1}$ and similarly $M_{0}=M_{*}$ at $t_{0}$. 
lower mass star. This is a correct description of mass loss which in practice is as accurate as the operator splitting procedure is accurate.

However, going from the $m_{r} / M_{0}$ to the $m_{r} / M_{*}$ grid while keeping $c_{i}$ as a function of $m_{r}$ implies interpolating. In practice, performing interpolations on $c_{i}$ during computations can introduce unwanted numerical diffusivity. It is relatively easy to show that these interpolations on $c_{i}$ resulting from mass loss can be avoided by introducing a local mass flux into the conservation equation, while keeping the grid constant (i.e. the grid points have the same values of $m_{r} / M_{*}$ in spite of $M_{*}$ varying from $M_{0}$ to $M_{1}$ ).

Let us consider the concentration on a grid point $\left(m_{r} / M_{*}\right)$ at $t_{1}$ as a function of the concentration on the same grid point at $t_{0}$. The grid points shift on the $m_{r}$ axis. Then

$c_{i}\left(m_{r 1}\right)=c_{i}\left(m_{r 0}\right)+\frac{\partial c_{i}}{\partial m_{r}} \Delta m_{r}$

which is a simple Taylor series development and where

$\Delta m_{r}=m_{r 1}-m_{r 0}$.

By definition, $m_{r 1}$ is the position on the $m_{r}$ axis, of the grid point $\frac{m_{r 0}}{M_{0}}$ after a $\Delta M_{\text {loss }}$ mass loss so that one may write:

$\frac{m_{r 1}}{M_{1}}=\frac{m_{r 0}+\Delta m_{r}}{M_{0}-\Delta M_{\text {loss }}}=\frac{m_{r 0}}{M_{0}}$

which can trivially be solved to give:

$\Delta m_{r}=-\frac{m_{r 0}}{M_{0}}\left(\Delta M_{\mathrm{loss}}\right)$.

Now we substitute Eq. (13) in Eq. (10), replace $\Delta M_{\text {loss }}$ by $-\dot{M} \times$ $\Delta t$ and simplify:

$c_{i}\left(m_{r 1}\right)=c_{i}\left(m_{r 0}\right)+\frac{\partial c_{i}}{\partial m_{r}} \frac{\mathrm{d} M_{*}}{\mathrm{~d} t} \frac{m_{r 0}}{M_{0}} \Delta t$.

The second term on the right has the same effect on $c_{i}$ as the introduction of a flux term ${ }^{5}$ (caused by mass loss $\times \frac{m_{r}}{M_{0}}$ ) in a conservation equation. The flux $4 \pi \rho r^{2} U_{W}$ given by equation

$$
-4 \pi \rho r^{2} U_{w}=\frac{\mathrm{d} M_{*}}{\mathrm{~d} t} \frac{m_{r}}{M_{*}} .
$$

must then be introduced in the conservation equation in order to take into account the effect of the $m_{r}$ shift caused by the peeling of surface layers while keeping the same grid as a function of $m_{r} / M_{*}$ when $M_{*}$ changes due to mass loss. This simple argument justifies the introduction of a mass flux to model the effect of mass loss on element separation in stellar atmospheres or envelopes (Vauclair 1975; Michaud et al. 1983; Alecian 1996).

For smaller mass loss rates, structural effects of mass loss are often negligible to the extent that only the shift of $c_{i}$ remains and mass loss may be viewed as a mass flux going through a star of constant mass (i.e. it is not necessary to change the stellar mass during evolution calculations). Consider the case of the present Sun. Assuming that it is constant in time, its current mass loss rate of $2 \times 10^{-14} M_{\odot} \mathrm{yr}^{-1}$ leads to a loss of $10^{-4} M_{\odot}$ up to the Sun's age which for most purposes corresponds to negligible structural changes. This is only $\sim 1 / 300$ of the mass of the superficial convection zone.

Since we are not certain about the nature of the winds at the surface of $\mathrm{A}$ and $\mathrm{F}$ stars, the present models do not take into account any energy dissipation which would be required to produce these winds.

\footnotetext{
5 Often abusively called a "wind" term in this context. It seems preferable to restrict the use of "wind" to the region above the photosphere.
}

\subsection{Stellar winds of $A$ and $F$ stars}

This study will use observed surface abundances to constrain mass loss since the stellar winds associated with $\mathrm{A}$ and $\mathrm{F}$ stars are not well known. The winds could be radiative, coronal, a combination of both or even completely negligeable. Comparisons with stellar wind models are difficult. Indeed, even the hottest A stars maintain a thin surface $\mathrm{H}$ convection zone, and stars as early as A7 (and possibly earlier, see Neff \& Simon 2008; Simon \& Landsman 1997) can support active coronas and chromospheres which could harbor solar type winds. It is also plausible that both mechanisms act simultaneously. A few properties of coronal and radiatively driven winds for $\mathrm{A}$ and $\mathrm{F}$ stars will be reviewed in the two following subsections, in so far as they relate to chemical separation.

\subsubsection{Coronal winds}

With simple physical considerations, it is possible to obtain an approximate value of the mass loss rate above which coronal winds are necessarily unseparated. For a spherically symmetrical mass loss, the wind velocity (Eq. (6) with $M_{r}=M_{*}$ ) may be compared to the maximal downward diffusion velocity (given by the gravitational settling velocity without any contribution from radiative accelerations). Equating the two gives an evaluation of the maximum mass loss that allows for separation to occur. One may then write:

$v_{w}=\frac{\frac{-\mathrm{d} M_{*}}{\mathrm{~d} t}}{4 \pi r^{2} \rho}=D_{i p} \frac{A_{i} g m_{p}}{k T}$.

which can be rewritten as:

$\frac{-1}{M_{*}} \frac{\mathrm{d} M_{*}}{\mathrm{~d} t}=\frac{2.4 \times 10^{-15} A_{i} T_{5}^{1.5}}{Z_{i}^{2}}\left[\mathrm{yr}^{-1}\right]$

$\left(T_{5}=T / 10^{5} \mathrm{~K}\right)$ where $A_{i}$ and $Z_{i}$ are the atomic mass and atomic number respectively of element $i$ and where the Coulomb term in the calculation of $D_{i p}$ is replaced by an approximate value. This applies both in the atmosphere and in the outer parts of the wind solution. For a solar type wind, assuming an isothermal corona of $T=10^{6} \mathrm{~K}$, one obtains a limiting mass loss rate $\simeq 10^{-13} M_{\odot} \mathrm{yr}^{-1}$ (see Michaud et al. 1987). The dependence on $T$ is valid when the gas is fully ionized. In the cooler atmospheres of early A stars (say at $10^{4} \mathrm{~K}$ ), He is mainly neutral, nevertheless the limiting mass loss rate is approximately the same in the atmosphere as in the corona since the diffusion coefficients are larger by a factor of 200-300 than those of ionized He (Michaud et al. 1978). The separation observed in the solar wind $\left(\dot{M} \simeq 10^{-14} M_{\odot} \mathrm{yr}^{-1}\right)$ agrees roughly with this analysis. As previously mentioned, observations show that elements which are neutral at $T=10^{4} \mathrm{~K}$ are $\simeq 2-3$ times less abundant in the corona than in the photosphere (Meyer 1985, 1996). Most agree that the separation occurs somewhere in the solar chromosphere immediately above the photosphere and could therefore have an impact on observed photospheric abundances. For instance, Geiss \& Buergi (1986, in their model 4) find that matter which arrives at $1 \mathrm{AU}$ has the same $\mathrm{He}$ to $\mathrm{H}$ ratio as at the wind's base. Since there is no net separation within the solar wind, it is precarious to infer that all coronal type winds are chemically differentiated. Moreover, wind structures in A star coronas would probably be quite different since many metals that are neutral in $\mathrm{G}$ and $\mathrm{F}$ star photospheres are ionized in A star photospheres. It is thus very difficult to compare the solar wind to other coronal winds, particularly for hotter stars. 


\subsubsection{Radiatively driven winds}

Babel (1995) found that for stars within $8000 \leq T_{\text {eff }} \leq 14000 \mathrm{~K}$, all radiatively driven winds must be fully separated. In these stars the Coulomb coupling is not sufficient to redistribute the momentum acquired by the heavier, radiatively accelerated elements onto the bound, more abundant $\mathrm{H}$ and $\mathrm{He}$ (Springmann \& Pauldrach 1992; Owocki \& Puls 2002; Krtička et al. 2003). Since only metals are ejected from the star, the mass loss rates are much smaller: between $10^{-16} M_{\odot} \mathrm{yr}^{-1}$ and $10^{-17} M_{\odot} \mathrm{yr}^{-1}$. However, the multicomponent hydrodynamical model put forth in Babel (1995) and Babel (1996) only considers an average metal, rather than solving for each metal individually, therefore metal-specific mass loss rates are not known. Furthermore, the interaction of radiatively driven winds with magnetic fields as well as convection is still poorly understood. This is particularly important for A stars, and the consequent uncertainties require us to be cautious before constraining our analysis with these results. For cooler stars, such as F stars, radiative accelerations are not believed to be able to generate significant mass loss (Abbott 1982).

Unglaub (2008) found that radiatively driven winds of sdB stars must be separated (accelerated metals cannot drag $\mathrm{H}$ and $\mathrm{He}$ ) if the mass loss is smaller than $10^{-12} M_{\odot} \mathrm{yr}^{-1}$ (10 times larger than the approximate value obtained in Sect.4.2.1). However, although the author's calculations are quite thorough, they are not complete in so far as some other poorly understood factors could play a significant role in determining wind properties. How does convection or magnetic fields, particularly flux tubes, affect the wind structure and velocity? Furthermore, the omission of line shadowing in the calculations could have a significant impact on the author's results ${ }^{6}$. In fact, the author stipulates in Sect. 6.2 of his article that including line shadowing could diminish $g_{\text {rad }}$ by a factor of 100 for the stronger photoshperic lines, thus leading to an overestimation of $\dot{M}$ by a factor of 10 .

\subsection{Unseparated vs. separated mass loss}

The object of this study is to constrain the effects of mass loss solely via observed abundance anomalies. To do so, we use simple wind models in order to minimize the arbitrariness of the analysis. Accordingly, most calculations were done assuming simple unseparated winds, although a few calculations were also carried out assuming separation in the wind in order to assess potential effects. Three cases of separated winds were considered: (1) only metals are ejected, (2) the separation mimics the solar wind with $\mathrm{H}$ treated as a high-FIP (First Ionization Potential; Meyer 1985) element and (3) the separation again mimics the solar wind but with $\mathrm{H}$ as a low-FIP element.

In case 1 , all metals are ejected with the same composition as the stellar surface, while $\mathrm{H}$ and $\mathrm{He}$ remain bound. For this scenario, mass loss rates were varied from $10^{-17}$ to $5 \times 10^{-16} M_{\odot} \mathrm{yr}^{-1}$ in order to account for the fact that only metals are leaving the star (around $2 \%$ of the superficial mass fraction).

The other scenarios (2 and 3 ) consider chemical separation in the solar wind as established by Meyer (1985), who found that elements with a FIP smaller than $9 \mathrm{eV}$ were approximately 4 times more abundant relative to hydrogen in the corona than

\footnotetext{
${ }^{6}$ Line shadowing occurs when the wind velocity is not sufficient to Doppler shift the wind's line centers away from flux attenuated photospheric line centers, thus reducing radiative accelerations. This is often true until the wind reaches the sonic point.
}

in the photosphere, while higher-FIP elements, including hydrogen, kept their photospheric abundances. Although this scenario is generally favored, Meyer (1996) questioned his own results a decade later by implying that instead of having overabundant low-FIP elements in the wind, higher-FIP elements, including $\mathrm{H}$, could be depleted in the corona ${ }^{7}$. Both configurations are investigated: case 2 has $\mathrm{H}$ as a bound high-FIP element, and case 3 has it as a low-FIP element. Our approach was to divide all elements into two groups: low-FIP elements (below $11 \mathrm{eV}$ ) and high-FIP elements ( $\mathrm{He}, \mathrm{C}, \mathrm{N}, \mathrm{O}, \mathrm{Ne}, \mathrm{Cl}$ and Ar). All low-FIP elements were depleted 4 times faster than high-FIP elements ${ }^{8}$.

Numerically, in cases (2) and (3), the destruction term in Eq. (9) was multiplied by a factor of 4 in the SCZ for all lowFIP elements. A weight term, which was continuously updated as concentrations changed in the SCZ, was added to the denominator for normally depleted high-FIP elements to account for the fact that their destruction is not, in this case, proportional to the total mass loss rate multiplied by individual concentration (Eqs. (7) and (9)) since their relative concentration in the photosphere is not the same as in the wind. The interior wind velocities are not affected since the $v_{\text {wind }}$ term does not depend on relative concentrations, but simply on the mass loss rate.

\section{Evolutionary models}

In Fig. 4, the evolution of $T_{\text {eff }}, L, \log g$, the depth of the surface convection zone as well as the surface Fe abundance are shown for a number of models. The chosen masses were selected to span the observed $T_{\text {eff }}$ range associated with AmFm stars and $\mathrm{Li}$ gap stars (Preston 1974; Boesgaard \& Tripicco 1986). The lower limit also corresponds to the least massive star on the main sequence for which we predict relatively large surface abundance anomalies (see Sect. 5.3). All models are shown for the same mass loss rate $\left(10^{-13} M_{\odot} \mathrm{yr}^{-1}\right)$. As will be seen in the next section, this mass loss rate has an effect, though very moderate, on the structure via abundance changes (the effect on He abundance for instance can increase the depth of the surface convection zone). This mass loss rate also allows surface abundance anomalies which are compatible with observed abundance anomalies for Am stars (see Sect. 5.3).

The main-sequence lifetime ranges from about $500 \mathrm{Myr}$ for the $2.5 M_{\odot}$ model to more than $3 \mathrm{Gyr}$ for the $1.3 M_{\odot}$ model. The Fe surface abundance is intimately coupled with the movement of the surface convection zone; for the models with the thinnest $\mathrm{SCZ}, \mathrm{Fe}$ is predicted to be overabundant over most of the MS lifetime (in the $2.5 M_{\odot}$ model by a factor of about 3 ).

All models of at least $1.5 M_{\odot}$ are marked by a rapid Fe abundance peak which occurs at the beginning of the main sequence. The rise of $X(\mathrm{Fe})$, which is related to the depth change of the surface convection zone as the star stabilizes (see Fig. 4f), is so rapid that it is not resolved in Fig. 4f. Similarly, there is a sharp spike towards the end of the MS for most models which is once again correlated with the sharp variation in SCZ depth (in this case the most important variation is for the $1.7 M_{\odot}$ model, which has a difference of a factor of 2.5 in Fe abundance within less than $100 \mathrm{Myr})$. As we will see in the following sections, these variations are larger when the mass loss rate is smaller. The HR diagram for these models is shown in Fig. 1. For clarity, only two

\footnotetext{
7 This question remains unanswered (see Feldman \& Widing 2003 for a complete review).

${ }^{8}$ Helium is assumed to have a 1:1 ratio with high-FIP elements, since the observations which suggest that He would have a ratio of 1:4 with these elements are questioned (see Feldman \& Widing 2003).
} 

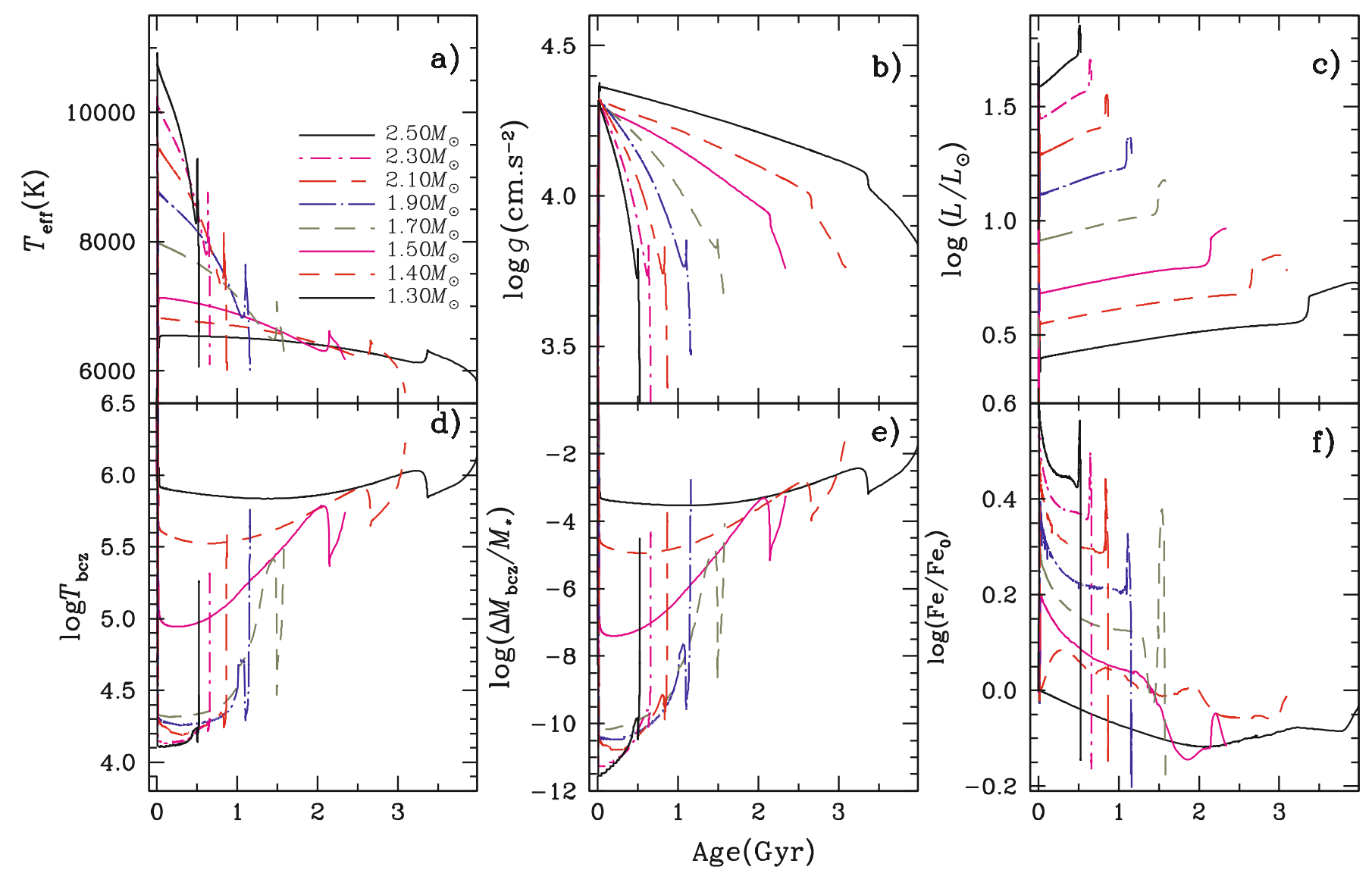

Fig. 4. Evolution of $T_{\text {eff }}$ (a), $\log g \mathbf{b}$ ), luminosity $\mathbf{c}$ ), temperature d) and mass at the bottom of the surface convection zone e), as well as the surface Fe abundance f) for selected masses that span the range of AmFm stars. All models are shown for a mass loss rate of $10^{-13} M_{\odot} \mathrm{yr}^{-1}$. The curves are identified in panel a). Vertically, they have the same order in panels a), c) and f), but are in reverse order in panels b), d) and e).

evolutionary tracks are shown from the initially homogeneous pre-main-sequence up to the subgiant branch (the others are shown from the beginning of the main-sequence, although their evolution is calculated from the pre-main-sequence). Diffusion and its effects on surface abundances appear well before the arrival on the main-sequence (a thorough investigation of diffusion on the pre-main-sequence will be discussed in Vick et al., in preparation).

\subsection{Radiative accelerations, internal abundance variations and structure}

In Figs. 5 and 6 the radiative accelerations as well as the corresponding spatial abundance variations for a few selected elements are shown for models of $1.5 M_{\odot}$ and $2.5 M_{\odot}$. These masses approximately correspond to the lower and higher $T_{\text {eff }}$ limits of AmFm stars.

The MS lifetime of the $1.5 M_{\odot}$ model is about 2 Gyr whereas the $2.5 M_{\odot}$ star has a MS lifetime of about $520 \mathrm{Myr}$ (see Fig. 4). The $1.5 M_{\odot}$ model with a mass loss rate of $1 \times 10^{-14} M_{\odot} \mathrm{yr}^{-1}$ (see bottom panel of Fig. 5) was stopped at $575 \mathrm{Myr}$ because the solution became numerically unstable.

Throughout most of the stellar envelope, the mass loss rate does not significantly affect the resulting $g_{\mathrm{rad}} \mathrm{s}$, as seen in the upper row of Fig. 5. However, if mass loss is small enough to permit iron peak accumulation below the SCZ, as is the case for the $1.50 \mathrm{~W} 1 \mathrm{E}-14$ model, then all $g_{\mathrm{rad}} \mathrm{S}$ will be affected by competition in the region where metals have accumulated.
We present in Sects. 5.1.1 and 5.1.2 approximate equations which will facilitate the interpretation of the detailed solutions shown in Figs. 5 and 6, which will be discussed in Sect. 5.2.

\subsubsection{The interior wind solution}

Consider the approximate solution to Eq. (7), in a regime for which the $\frac{\partial c}{\partial t}$ term is small compared to the others (which is true over most of the MS lifetime). Then one may write:

$\nabla \cdot\left[-\rho D \nabla \ln c+\rho\left(\boldsymbol{U}+\boldsymbol{U}_{w}\right) c\right]=0$

if nuclear terms are negligeable, which is true for the stellar envelope, and $S_{\mathrm{w}}=0$, which is true outside the surface convection zone. In one dimension, and in the absence of turbulence (so that $D$ becomes $D_{12}$, the diffusion coefficient in a hydrogen and helium background) Eq. (18) becomes:

$\mathcal{F}(r)=r^{2}\left[-\rho D_{12} \frac{\partial c}{\partial r}+\rho\left(U+U_{\mathrm{w}}\right) c\right]=$ cst.

where $\mathcal{F}(r)$ is constant as a function of $r$ and $U$ is approximately given by Eq. (9) from Michaud et al. 1983, with a slight correction ${ }^{9}$ :

$$
\begin{array}{r}
U \simeq v_{\mathrm{D}}=D_{12}\left[-\left(A-\frac{Z}{2}-\frac{1}{2}\right) \frac{m_{\mathrm{p}} g}{k T}\right. \\
\left.+\frac{m_{\mathrm{p}} A g_{\mathrm{rad}}}{k T}+\alpha_{T} \frac{\partial \ln T}{\partial r}\right] .
\end{array}
$$

\footnotetext{
$9 \frac{m_{\mathrm{p}}}{k T}$ had been erroneously forgotten in the 2 nd and 3rd terms.
} 

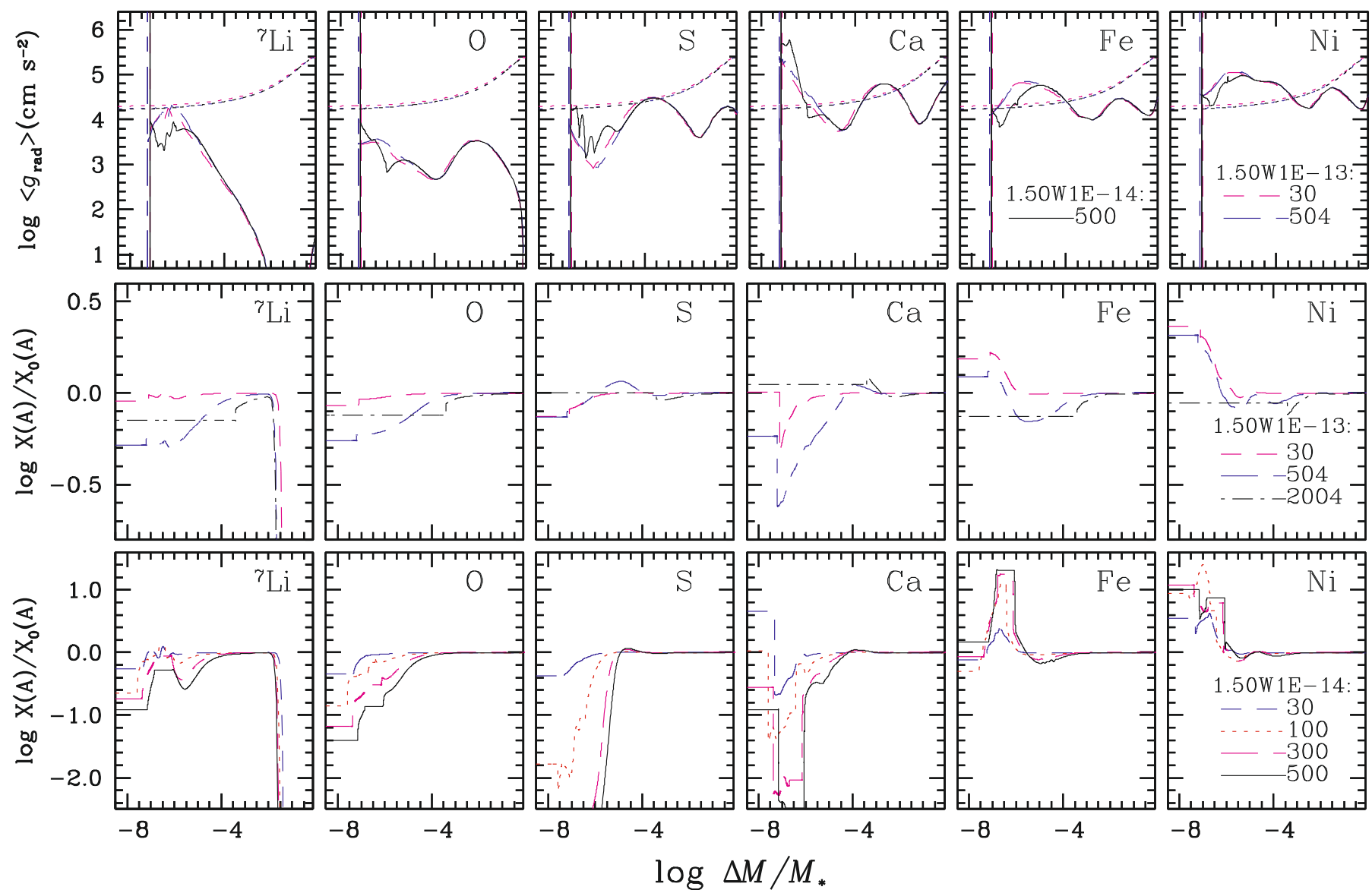

Fig. 5. (Top row) Radiative accelerations (solid line) and local gravity (dotted line) for a few selected elements in a $1.5 M_{\odot}$ model with two different mass loss rates. For the 1.50W1E-13 model, the radiative accelerations are shown for 2 ages ( 30 and $503 \mathrm{Myr}$ ), while they are only shown at $500 \mathrm{Myr}$ for the $1.50 \mathrm{~W} 1 \mathrm{E}-14$ model. The vertical lines show the position of the bottom of the surface $\mathrm{H}-\mathrm{He}$ convection zone. The corresponding internal abundance variations at different ages (in Myr) are shown for both the 1.50W1E-13 model (middle row) and the 1.50W1E-14 model (bottom row).

Here $A$ and $Z$ are the atomic mass and charge number respectively, and $\alpha_{T}$ is the thermal diffusion coefficient ${ }^{10}$. Since the $g$ and $g_{\text {rad }}$ terms are multiplied by $A$ while the abundance gradient term (in Eq. (19)) is not, only very large abundance gradients can affect the diffusion velocity. Therefore, the internal abundance distribution approximately satisfies the relation:

$\mathcal{F}(r)=$ cst. $\simeq r^{2} \rho\left(U+U_{\mathrm{w}}\right) c$

which expresses the conservation of particle flux throughout the stellar envelope. In this discussion, for the purpose of illustration, one may also neglect the term involving $\frac{\partial \ln T}{\partial r}$ because it is not dominant in the relevant regions of the envelope (although it is always included in our calculations).

The implications of flux conservation are illustrated in Fig. 7 for three atomic species ( $\mathrm{Ca}, \mathrm{Mn}$ and $\mathrm{Ni}$ ) from the $2.5 \mathrm{M}_{\odot}$ model of Fig. 6. For all three species, the flux is always positive as may be seen from the top row. While $g_{\mathrm{rad}}(\mathrm{Ca})$ is smaller than gravity at some mass shells (middle row), the downward diffusion velocity of $\mathrm{Ca}$ is never larger than the wind velocity (see Fig. 10) so that it is dragged by the wind toward the surface. In general, as long as the absolute value of the diffusion velocity is smaller than the wind velocity, the concentration simply

\footnotetext{
10 This equation is not used for the calculations. For more details see Turcotte et al. (1998b).
}

increases in order to conserve the flux (as implied by Eq. (21) $)^{11}$ This equation may then be used here for all three species. As may be seen from the bottom row, after $3 \mathrm{Myr}$, the concentration has adjusted only down to $\Delta M / M_{*} \simeq 3 \times 10^{-7}$. Therefore, below this depth, the local flux (top row) mainly reflects the local $g_{\text {rad }}$. After about 10 Myr however, the abundance has adjusted to carry the flux which arrives from deep inside the star (down to $\log \Delta M / M_{*} \simeq-6$ ). This may be evaluated using $\Delta M \simeq \dot{M} \times t$ for a mass loss rate of $10^{-13} M_{\odot} \mathrm{yr}^{-1}$. After $100 \mathrm{Myr}$, the concentration has adjusted to the flux down to $\log \Delta M / M_{*} \simeq-5$ and the concentration becomes the mirror image of $g_{\text {rad }}$ from the surface to that depth as may be seen by comparing the middle and bottom rows. There are $\mathrm{Ca}$ overabundances at $\log \Delta M / M_{*}=-8.5$ and -4.5 , which occur at $g_{\mathrm{rad}}(\mathrm{Ca})$ minima (see also Fig. 6), while the minimum abundance occurs at the maximum of $g_{\mathrm{rad}}(\mathrm{Ca})$. However, if the wind velocity were not larger than the settling velocity, a gradient could develop to conserve the flux. If this gradient cannot become large enough to conserve flux, then the $\partial c / \partial t$ term (in Eq. (7)), which is required to be zero in the kinematic approximation becomes important, and the approximation leading to Eq. (21) becomes invalid.

The net elemental flux toward the surface quickly becomes nearly constant in time once the local abundance has adjusted

\footnotetext{
11 This resembles the results for oxygen which are discussed in Landstreet et al. (1998).
} 


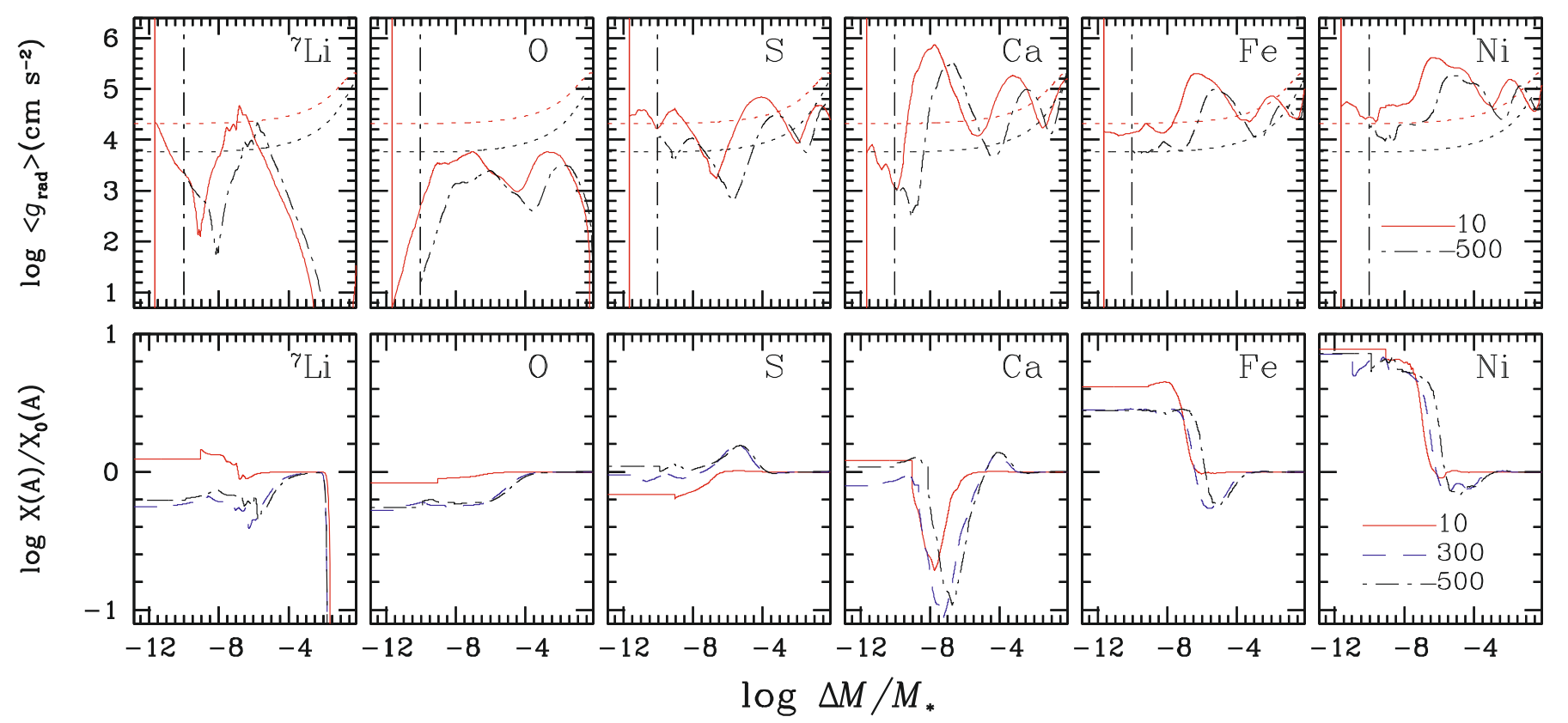

Fig. 6. Top row Radiative accelerations (solid line) and local gravity (dotted line) in a $2.5 M_{\odot}$ model with a mass loss rate of $1 \times 10^{-13} M_{\odot} \mathrm{yr}^{-1}$. The corresponding internal abundance variations are shown at three ages (bottom panel). All curves are identified by their age (in Myr). The vertical lines show the position of the bottom of the surface $\mathrm{H}$ convection zone.

itself to conserve the flux. The remaining slow variation in time of the flux comes from the variation of $g_{\text {rad }}$ where the matter originated, deep in the star, at $\Delta M \simeq \dot{M} \times t$.

\subsubsection{The kinematic approximation}

By following the analysis of Michaud \& Charland (1986), the surface abundance of elements which are pushed upwards by $g_{\mathrm{rad}}$ and/or dragged by the wind throughout the stellar envelope can be approximated by using a simple kinematic equation so long as the evolutionary effects and the contribution of $\frac{\partial c}{\partial t}$ are small (i.e. there is no significant accumulation). The problem of determining surface abundances thereby reduces to a kinematic problem. The only required quantity is the local velocity and, consequently, the radiative acceleration. Elements which originated at $r_{1}$ at $t=0$ arrive at $r_{\mathrm{cz}}$, the radius at the bottom of the surface convection zone at a time $t_{1}$ given by:

$t_{1}=\int_{r_{1}}^{r_{\mathrm{cz}}} \frac{\mathrm{d} r}{v_{\mathrm{w}}+v_{\mathrm{D}}}$.

Because of flux conservation, the flux of an element $A$ entering the convection zone at $t_{1}$ is then given by:

$\phi\left(t_{1}, A\right)=c_{0}\left(A, r_{1}\right)\left(v_{\mathrm{D}, r_{1}}+v_{\mathrm{w}, r_{1}}\right) \rho_{r_{1}} \frac{r_{1}^{2}}{r_{\mathrm{cz}}^{2}}$,

where $c_{0}\left(A, r_{1}\right)$ is the initial abundance of $A$ at $r_{1}$ and $v_{\mathrm{D} 1}, v_{\mathrm{w} 1}$ and $\rho_{1}$ are evaluated at $r_{1}$. This is valid for the region above which $v_{\mathrm{w}}+v_{\mathrm{D}}>0$ and for $t<t_{0 \mathrm{~A}}$, the time required for the element $A$ to migrate from the point where $v_{\mathrm{w}}+v_{\mathrm{D}} \simeq 0$ to the bottom of the convection zone. Since diffusion timescales are much longer than convective mixing time scales, the convection zone is assumed thoroughly mixed up to the bottom of the wind forming region. Within the context of unseparated mass loss, the evolution of the abundance of $A$ in the surface convection zone, $c_{\mathrm{cz}}(A)$ can then be approximated by:

$M_{\mathrm{cz}} \frac{\partial c_{\mathrm{cz}}(A)}{\partial t}=-\dot{M} c_{\mathrm{cz}}(A)+4 \pi r_{\mathrm{cz}}^{2} \phi\left(t_{1}, A\right)$, where $M_{\mathrm{cz}}$ and $r_{\mathrm{cz}}$ are respectively the mass of the superficial convective zone and the radius at the bottom of this convection zone. Equation (24) is not used for our calculations, but is used to interpret the results.

\subsection{Discussion: internal variations}

By comparing Figs. 5 and 6, it is clear that the same mass loss rate leads to quite different internal concentration variations in stars of different mass. There are two main reasons for this. First, in the more massive star, the surface convection zone is much thinner, therefore the radiative zone is extended upward into regions where the diffusion timescales are much shorter. Second, because of the $T_{\mathrm{eff}}{ }^{4}$ dependence of the photon flux, most $g_{\mathrm{rad}} \mathrm{S}$ are stronger in more massive stars.

For both models, diffusion mainly affects the outer $10^{-3}$ of the star's mass; the point above which the effects of atomic diffusion become visible in Figs. 5 and 6 will be defined as the point of separation. Atomic diffusion can also act deeper in the star (e.g. in the core), though in A and F main-sequence stars, its effects are much smaller below than above the point of separation.

\subsubsection{The $1.5 M_{\odot}$ models}

For the $1.5 M_{\odot}$ model with a mass loss rate of $1 \times 10^{-13} M_{\odot} \mathrm{yr}^{-1}$ (henceforth designated 1.50W1E-13 for short), internal anomalies are small. Iron reaches an overabundance of 1.5 , and nickel of 2.0. Lithium, oxygen and calcium decrease from the point of separation up to the bottom of the surface convection zone. Differences in $g_{\text {rad }}$ lead to differences in their behavior. Due to a strong $g_{\text {rad }}$ immediately below the surface $\mathrm{CZ}, \mathrm{Ca}$ is most underabundant in that region, since $\mathrm{Ca}$ is pushed into the convection zone by both $g_{\mathrm{rad}}$ and the wind while little arrives from below because of the minimum in its $g_{\text {rad }}$ at $\log \Delta M / M_{*} \sim-4.5$. At $500 \mathrm{Myr}$, its underabundance reaches a factor of 4 while the $\mathrm{Li}$ underabundance below the SCZ is about -0.3 dex. 
M. Vick et al.: AmFm and lithium gap stars

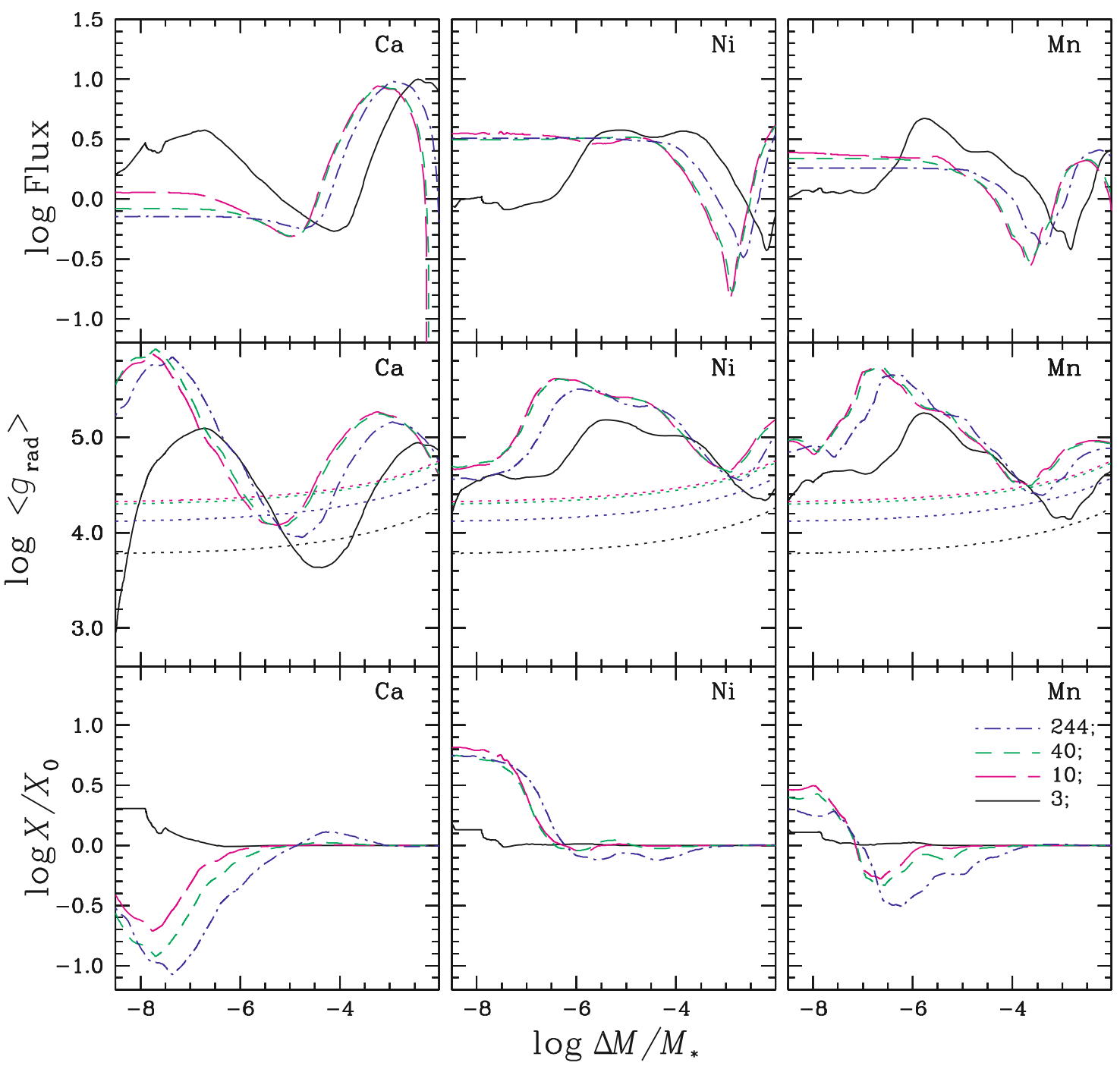

Fig. 7. Comparison of the normalized local flux with radiative accelerations and internal abundances for 3 elements at 4 different ages (in Myr) for a $2.5 M_{\odot}$ model with a mass loss rate of $10^{-13} M_{\odot} \mathrm{yr}^{-1}$.

In the lower row of Fig. 5, one sees that when the wind is 10 times smaller, the internal variations are much stronger since the advection by $v_{\text {wind }}$ is not strong enough to prevent elemental accumulation. Lithium has an interesting behavior: it has a local maximum where its $g_{\text {rad }}$ equals gravity. The underabundances of $\mathrm{Li}$ and $\mathrm{O}$ drop to -0.95 and -1.35 dex respectively, while underabundances greater than 2 dex are reached for $\mathrm{S}$ and Ca. Through Eq. (21), when $U_{\mathrm{w}}$ is smaller, the change in $U$ due to $g_{\text {rad }}$ has a larger effect on concentration. This is particularly true for Fe: due to a dip in $g_{\text {rad }}$ (see Fig. 5), it accumulates near $\log \Delta M / M_{*}=-6.5$ (where $T=200000 \mathrm{~K}$ ). The overabundances of $\mathrm{Fe}$ and $\mathrm{Ni}$ reach 1.25 and 1.45 dex respectively. The implications of this accumulation are analyzed in Fig. 8. The local opacity bump in the region between $\log \Delta M / M_{*} \simeq 6.0-6.8$ leads to the appearance of an iron peak convection zone $\left(\nabla_{r}-\nabla_{\text {ad }}\right.$ changes sign) at around $70 \mathrm{Myr}$ which survives until the end of the simulation. $\mathrm{Fe}$ and $\mathrm{Ni}$ reinforce each other. Only the $10^{-14} M_{\odot} \mathrm{yr}^{-1}$ model becomes convective; doubling the mass loss rate reduces the $\mathrm{Fe}$ accumulation enough for the iron peak convection zone not to appear.

In fact, a noteworthy transition occurs in internal solution types between the 1.50W1E-14 and 1.50W2E-14 models (Fig. 8). In the 1.50W1E-14 model, Fe is overabundant where
$g_{\mathrm{rad}}(\mathrm{Fe})>g$, whereas $\mathrm{Fe}$ is minimal at the $g_{\mathrm{rad}}(\mathrm{Fe})$ maximum in the 1.50W2E-14 model (similarly, Ca is minimal at the $g_{\mathrm{rad}}(\mathrm{Ca})$ maximum in the $2.50 \mathrm{~W} 1 \mathrm{E}-13$ model shown in Fig. 7). This is due to the relationship between $v_{\text {wind }}$ and the settling velocities for each individual species; if the settling velocity dominates locally, the element will accumulate locally, and the solution will behave as does Fe in the $1.50 \mathrm{~W} 1 \mathrm{E}-14$ model. When $v_{\text {wind }}$ dominates throughout the envelope, the solution is determined by flux conservation, as shown in Fig. 7. This transition separates the solution in which the surface abundances reflect matter which is advected from deep inside the star $\left(v_{\text {wind }}>v_{\text {sett }}\right)$ from the solution in which the surface reflects variations at the bottom of the SCZ (as in the models of Watson 1971 and Alecian 1996). This transition occurs only for elements whose $g_{\text {rad }}$ has a minimum smaller than gravity close to the bottom of the SCZ.

A large enough mass loss rate also affects the position of the bottom of the surface $\mathrm{H}-\mathrm{He}$ convection zone by keeping $\mathrm{He}$ from sinking. Since it also modifies the accumulation of metals, mainly iron and nickel, its effect is complex: the relative position of the bottom of the convection zone for the three mass loss rates in Fig. 8 is different at 70 and 500 Myr.

The inversion of the molecular weight gradient (bottom row of Fig. 8 at $500 \mathrm{Myr}$ ), which eventually follows the appearance 

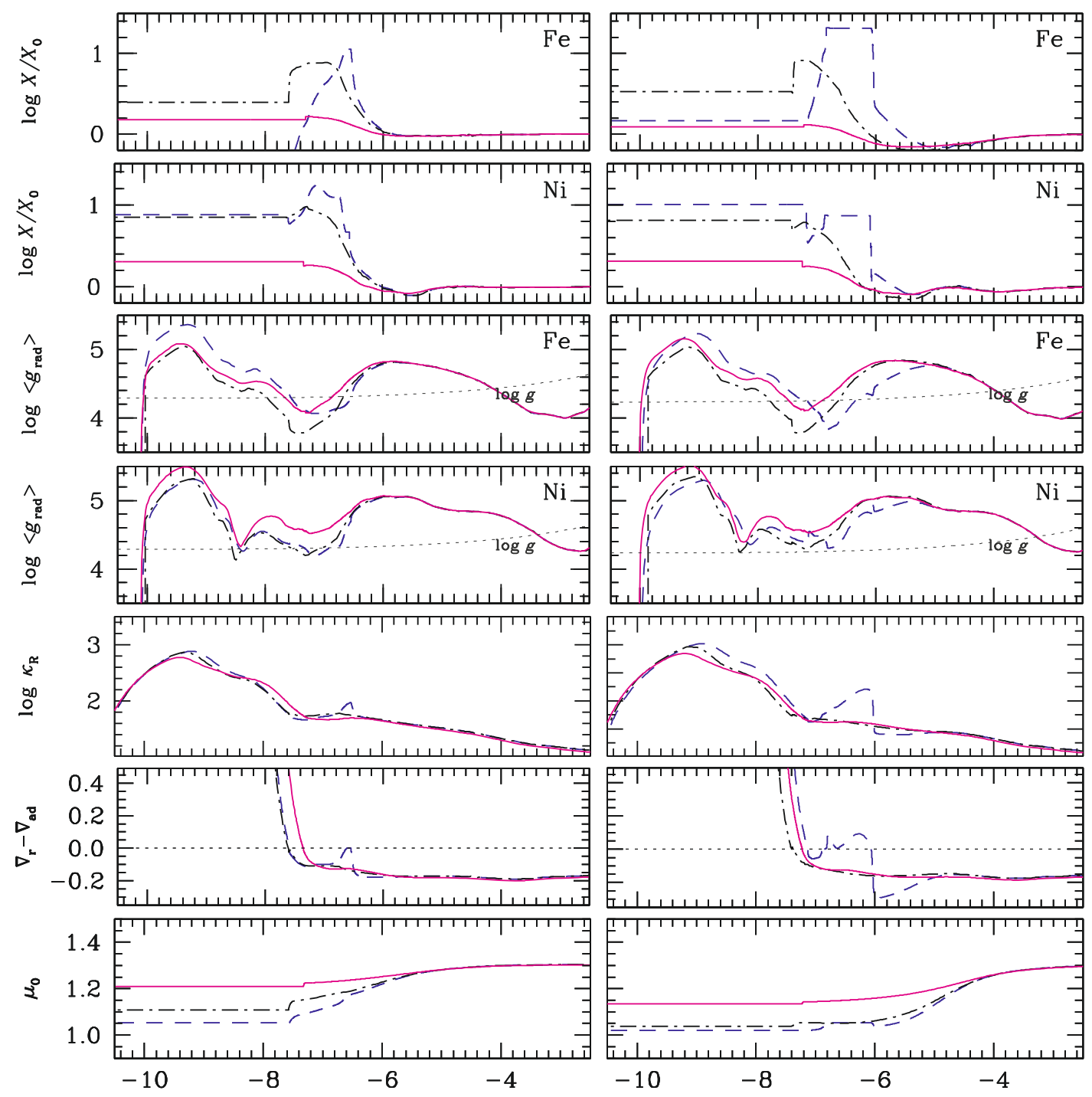

$\log \Delta \mathrm{M} / \mathrm{M}_{*}$

Fig. 8. Internal abundances $(\mathrm{Fe}, \mathrm{Ni})$, radiative accelerations $(\mathrm{Fe}, \mathrm{Ni})$, Rosseland opacity, the difference between the radiative and adiabatic temperature gradients as well as the mean molecular weight per nucleus for three $1.5 M_{\odot}$ models with different mass loss rates $\left(10^{-14} M_{\odot} \mathrm{yr}^{-1}\left(\mathrm{dashed}^{-}\right.\right.$ line), $2 \times 10^{-14} M_{\odot} \mathrm{yr}^{-1}$ (dot-dashed line) and $10^{-13} M_{\odot} \mathrm{yr}^{-1}$ (solid line)) at $70 \mathrm{Myr}$ (left column) and $500 \mathrm{Myr}$ (right column). At this mass, the Fe peak convection zone (between $\log \Delta M / M_{*}=-6$ and -7 ) only appears for the mass loss rate of $10^{-14} M_{\odot} \mathrm{yr}^{-1}$. The H-He convection zones remain linked for all mass loss rates.

of the iron accumulation around $200000 \mathrm{~K}$, has been suggested to affect the presence of the iron peak convection zone (Théado et al. 2009). In the present calculations however, the iron peak convection zone appears (as seen at $70 \mathrm{Myr}$ ) before the inversion appears so that while the size of the convection zone could be affected by the $\mu$ gradient inversion, its appearance cannot be affected. This will be further discussed in Sect. 8 .

\subsubsection{The $2.5 M_{\odot}$ models}

Large overabundances of iron peak elements are obtained in the $2.5 M_{\odot}$ models (Fig. 6): $\mathrm{Ni}$ and Fe are overabundant by factors of 7.5 and 4 respectively. Calcium on the other hand is found to be up to 10 times underabundant around $\log \Delta M / M_{*}=-6.5$, where its $g_{\text {rad }}$ is near a maximum. This may seem counter intuitive but is a consequence of Eq. (21). Since the flux is conserved, where $v_{\mathrm{D}}$ is large and positive, $c$ decreases, which is precisely what happens for Ca just below the convection zone (see Figs. 6 and 7 and the last two paragraphs of Sect. 5.1.1).

Wind and diffusion velocities are compared in Fig. 10. The wind velocity decreases more rapidly than the diffusion velocity as $\log \Delta M / M_{*}$ increases. For instance, the absolute value of the ${ }^{4} \mathrm{He}$ settling velocity is 50 times smaller than the larger of the two wind velocities at $\log \Delta M / M_{*}=-12$ but equals it at $\log \Delta M / M_{*}=-4.5$. For smaller mass loss rates the settling velocity dominates closer to the surface. Calcium has an upward diffusion velocity over the interval $-9<\log \Delta M / M_{*}<-6$ and it is up to ten times larger than the wind velocity. Fe and $\mathrm{Li}$ have smaller upward diffusion. Those velocities determine local concentration via Eq. (21) (see the end of Sect. 5.1.1).

Figure 9 allows an analysis of the effect of varying mass loss rates on surface convection zones and to distinguish the effects of mass loss from those of turbulence (see Sect. 6). The abundances and $g_{\mathrm{rad}}$ of both $\mathrm{Fe}$ and $\mathrm{Ni}$ are presented since they are the main 

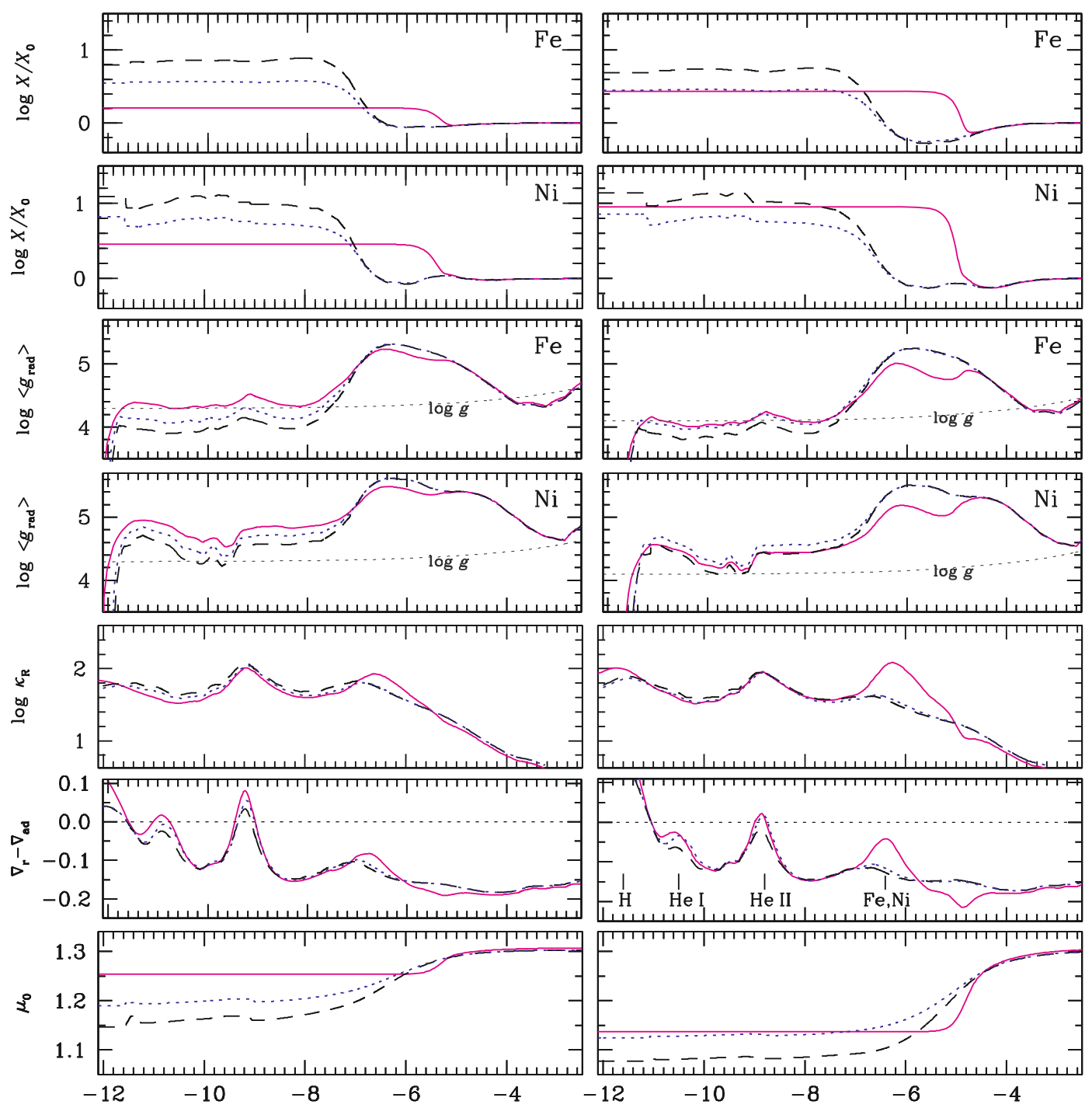

$\log \Delta \mathrm{M} / \mathrm{M}_{*}$

Fig. 9. Internal abundances $(\mathrm{Fe}, \mathrm{Ni})$, radiative accelerations $(\mathrm{Fe}, \mathrm{Ni})$, Rosseland opacity, the difference between the radiative and adiabatic temperature gradients as well as the mean molecular weight per nucleus for two $2.5 M_{\odot}$ models with different mass loss rates $\left(10^{-13} M_{\odot} \mathrm{yr}^{-1}\right.$ (dotted line) and $5 \times 10^{-14} M_{\odot} \mathrm{yr}^{-1}$ (dashed line)) as well as a model with turbulence (solid line) at $30 \mathrm{Myr}$ (left column) and around $250 \mathrm{Myr}$ (right column). The maxima in the $\nabla_{r}-\nabla_{\text {ad }}$ row are mainly due to opacities from $\mathrm{H}$, He I, He II and $\mathrm{Fe} / \mathrm{Ni}$ from left to right respectively.

contributors to the appearance of the iron peak convection zones in $1.5 M_{\odot}$ models. In $2.5 M_{\odot}$ models however, iron convection zones do not appear for the two mass loss rates considered. The $\mathrm{Fe}$ and $\mathrm{Ni}$ abundances remain slightly below solar where $g_{\mathrm{rad}}(\mathrm{Fe})$ and $g_{\mathrm{rad}}(\mathrm{Ni})$ are largest so that they do not lead to an increase of opacity where they could contribute most to opacity. The wind velocity is ten times larger than the diffusion velocity of Fe for $\log \Delta M / M_{*} \geq-8$ so that Eq. (21) forces the solution to be nearly constant over that range. This is to be contrasted with their behavior in $1.5 M_{\odot}$ models.

Even a small mass loss rate difference can have important effects on the stellar structure in $2.5 M_{\odot}$ models. For both mass loss rates considered, the He I convection zone disappears early in the evolution, at around $30 \mathrm{Myr}$ (see the sixth row of Fig. 9). The He II convection zone on the other hand only disappears in the model with the smaller mass loss rate, the 2.50W5E-14 model, at around $200 \mathrm{Myr}$. This is because the inward diffusion velocity of $\mathrm{He}$ dominates the wind velocity at a shallower depth than in the 2.50W1E-13 model (see Fig. 10), so that a more pronounced He underabundance develops to conserve the flux.

\subsection{Surface abundance variations}

\subsubsection{The $1.5 M_{\odot}$ models}

The evolution of surface abundances is shown for several $1.5 M_{\odot}$ models in the upper two rows of Fig. 11. For the 1.50W1E-12 model, the largest anomaly encountered, which is for $\mathrm{Ni}$, reaches a mere $0.05 \mathrm{dex}$, and it is quickly flattened after a brief period. Such a mass loss rate effectively wipes out any surface effects of chemical separation. In the $1.5 \mathrm{~W} 1 \mathrm{E}-14$ model, some of the calculated underabundances are very large (greater than 2 dex for $\mathrm{S}$ as well as $\mathrm{C}$ and $\mathrm{Si}$, which are not shown). With the exception of $\mathrm{Fe}$, all iron peak elements are overabundant throughout the simulation. Both $\mathrm{Ni}$ and $\mathrm{Mn}$ become more than 1 dex overabundant at around $500 \mathrm{Myr}$ and $50 \mathrm{Myr}$ respectively.

Iron surface abundances are particularly interesting for this model since it is the only one to have an iron surface underabundance which spans $500 \mathrm{Myr}^{12}$. This is due to the

12 We may assume however that if the simulation had not encountered significant instabilities, the shown iron abundance trend would have continued and iron would have become overabundant. 


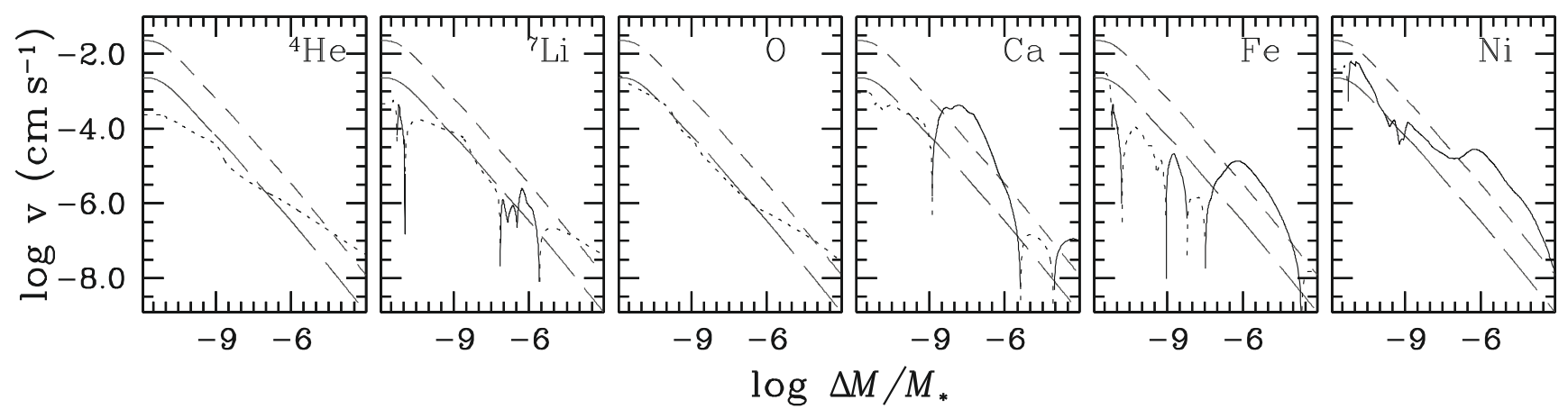

Fig. 10. Wind velocities (long dashed line: $10^{-14} M_{\odot} \mathrm{yr}^{-1}$; dashed line: $10^{-13} M_{\odot} \mathrm{yr}^{-1}$ ) and diffusion velocities (solid when positive, towards the surface, and dotted when negative) of a few selected elements in two $2.50 M_{\odot}$ models at 300 Myr. For most species, wind velocities decrease more rapidly inwards than diffusion velocities.
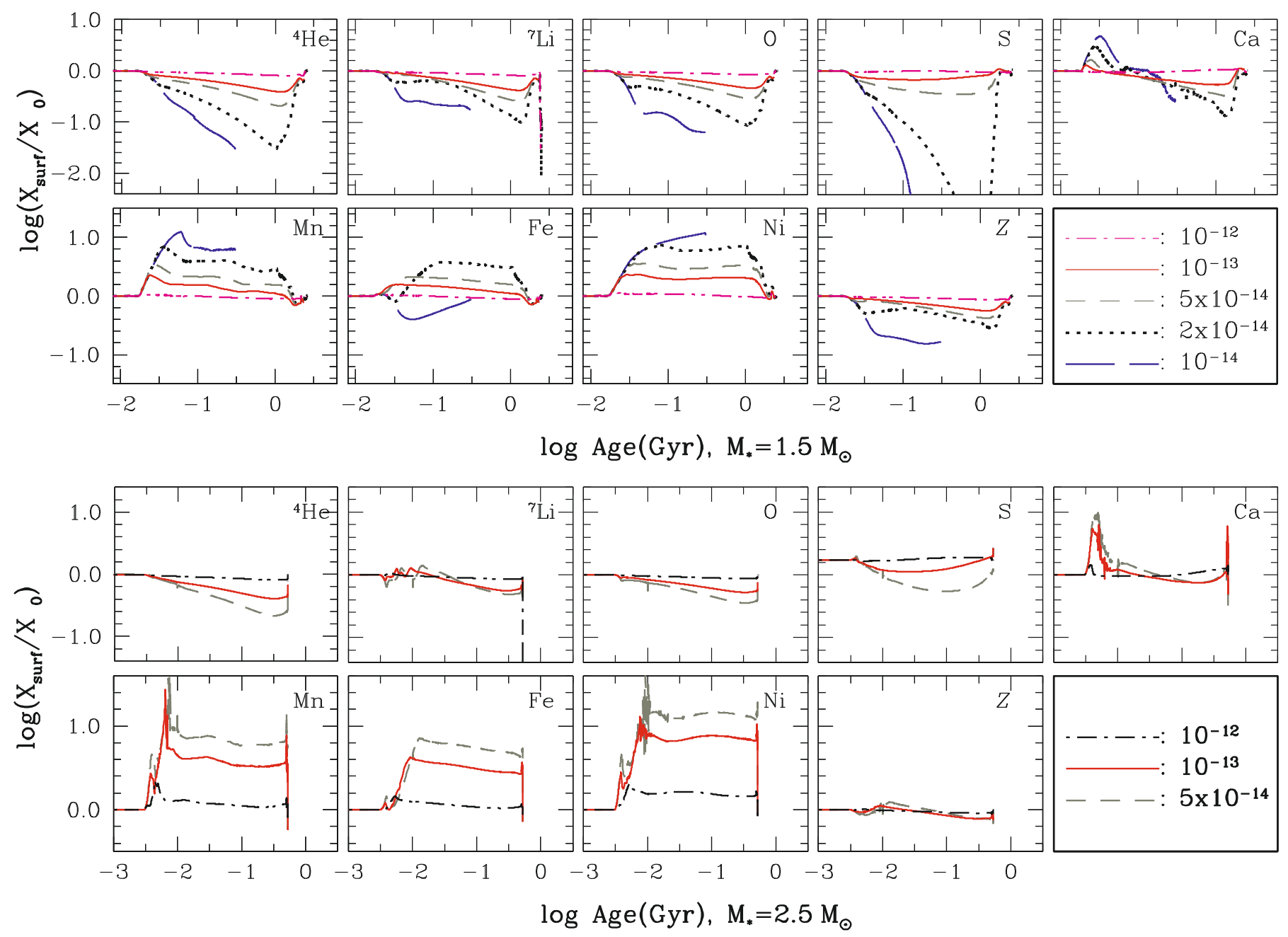

Fig. 11. Surface abundance variations for five $1.50 M_{\odot}$ models (top 2 rows) as well as three $2.50 M_{\odot}$ (lower 2 rows) models with $Z_{0}=0.02$ and varying amounts of mass loss (in $M_{\odot} \mathrm{yr}^{-1}$ ). The model was ended at around $550 \mathrm{Myr}$ due to numerical instabilities which were caused by the severe underabundances of many elements (of which $\mathrm{S}$ is shown). It is also the only model which leads to the appearance of a separate iron peak convection zone. In the bottom panel, the two models with smaller mass loss rates lead to a surface metallicity which has a maximum around $10 \mathrm{Myr}$ before falling below $Z_{0}$ around $100 \mathrm{Myr}$.

accumulation of iron under the surface convection zone, and the subsequent appearance of an iron peak convection zone (see Fig. 8). Although not shown here, a separate iron peak convection zone is expected for all simulations for masses between $1.47 M_{\odot}$ and $3.0 M_{\odot}$ when the mass loss rate is equal to or below $\sim 10^{-14} M_{\odot} \mathrm{yr}^{-1}$.
The 1.50W2E-14 and 1.50W5E-14 models generate surface iron overabundances of about 0.5 and 0.35 respectively at 300 Myr. The calcium abundance is particularly interesting since for all models, an overabundance is predicted at the beginning of the main sequence evolution followed by an underabundance. The underabundance is present over a much larger fraction of 


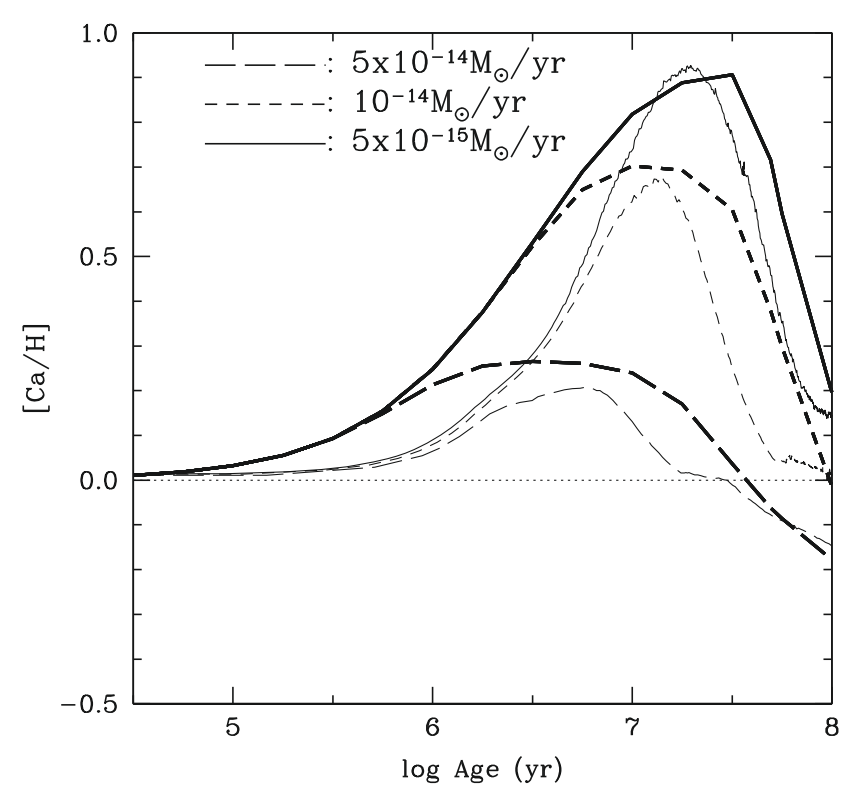

Fig. 12. Evolution of Ca surface abundances for $1.5 M_{\odot}$ models with different mass loss rates. The calculations from Alecian (1996) are shown in bold.

the evolution than the overabundance. The Ca calculations are compared in Fig. 12 with those carried out by Alecian (1996). His calculations were undertaken in static stellar models; therefore, evolutionary effects were not included. There are also slight differences in $T_{\text {eff }}$ between our calculations ( $T_{\text {eff }} \simeq 7200 \mathrm{~K}$ at the beginning of diffusion but decreases as the star evolves) and his simulations $\left(T_{\text {eff }}=7500 \mathrm{~K}\right)$. There are also differences in $g_{\text {rad }}(\mathrm{Ca})$ : his calculated $g_{\text {rad }}(\mathrm{Ca})$ has a peak which is 10 times smaller than obtained in a model of similar $T_{\text {eff }}$ with our code. Results are compared in Fig. 12 for three mass loss rates. In all three cases, the maximum anomaly as well as the overall behavior of the curves correspond well. The overabundance peaks are however not quite so wide in our calculations as in his. The agreement seems satisfactory.

\subsubsection{The $2.5 M_{\odot}$ models}

In the $2.5 M_{\odot}$ models (lower two rows of Fig. 11), the surface abundances of heavier metals (say $Z>17$ ) can be characterized by two distinct episodes: a steep abundance spike, followed by a smooth decline. The initial peaked episode around $10 \mathrm{Myr}$ shows matter which was above $\log \Delta M / M_{*} \simeq-6$ at the onset of diffusion, advected to the surface by the stellar wind.

As discussed at the end of Sect.5.1.1, the initial flux distribution reflects $g_{\mathrm{rad}}$ since, originally, the concentration is the same throughout the envelope; then, as time elapses, the internal abundances naturally evolve in such a way that flux is conserved throughout the envelope (see Fig. 7). In so far as the $\partial c / \partial t$ term is negligible (i.e. flux is conserved), the surface reflects the point in the initial flux distribution which is dragged by $v_{\text {wind }}$. Therefore, when the initial internal flux profile of an element has important variations near the surface due to strong variations in $g_{\text {rad }}$ (as is the case for $\mathrm{Ca}$ and $\mathrm{Ni}$, see Figs. 6 and 7), these variations appear on the surface in a time which is related to the mass loss rate.

Quantatively, in the 2.50W1E-13 model (solid line), a given element's surface abundance at $100 \mathrm{Myr}$ depends on the initial flux variations (caused by $g_{\mathrm{rad}}$ variations at $t=0$ ) ${ }^{13}$ at a depth of $\Delta M \simeq \dot{M} \times t=10^{-13} M_{\odot} \mathrm{yr}^{-1} \times 10^{8} \mathrm{yr} \simeq 10^{-5} M_{\odot}$. In Fig. 11, the slight bump in surface Ni abundance around $100 \mathrm{Myr}$ for the 2.50W1E-13 model reflects the small bump in the initial flux distribution of $\mathrm{Ni}$ around $\log \Delta M / M_{*} \simeq-5$ (see Fig. 7).

The previous example is an application of Eq. (22) and shows that the time it takes for a given internal variation to reach the surface is inversely proportional to the mass loss rate. This is further illustrated by the fact that the surface Ni abundance peak around $10 \mathrm{Myr}$ appears earlier as the mass loss rate increases (see Fig. 11). The dilution by the convection zone (Eq. (24)) is relatively small because it has a relatively small mass. Also, the rapid variations seen around this $\mathrm{Ni}$ abundance peak reflect the many internal flux variations which were above $\log \Delta M / M_{*} \simeq-6$ at $t=0$. For a mass loss rate of $10^{-13} M_{\odot} \mathrm{yr}^{-1}$, these variations all reach the surface within $10^{7} \mathrm{yr}$.

As a consequence, the nearer an internal flux variation is to the surface at $t=0$, the quicker it appears at the surface, and the quicker it disappears. A flux variation that spatially spans from the surface to $\log \Delta M / M_{*}=-7$ at $t=0$ appears and disappears at the surface in less than one million years for a mass loss rate of $10^{-13} M_{\odot} \mathrm{yr}^{-1}$.

The amplitude of the surface variations also depends on the mass loss rate; as the mass loss rate increases, each internal variation of the flux at $t=0$ manifests itself at the stellar surface with a smaller amplitude since more enriched/depleted matter is evacuated from the SCZ (Eq. (24)). This is illustrated by the decreasing amplitude of the initial surface abundance spikes for $\mathrm{Mn}$ and Ni as the mass loss rate increases (Fig. 11).

The conservation of the flux down to $\log \Delta M / M_{*} \simeq-5.5$ to -6.5 (depending on the age and mass loss rate) has another important consequence: diffusion between the surface convection zones (i.e. between the $\mathrm{H}$ and either of the two $\mathrm{He}$ convection zones) has practically no influence on the surface abundances. Matter which originates from between the $\mathrm{H}$ and $\mathrm{He}$ convection zones $\left(\log \Delta M / M_{*} \simeq-10\right)$ appears at the surface within $10^{3}$ years for a mass loss rate of $10^{-13} M_{\odot} \mathrm{yr}^{-1}$. The detailed calculation of chemical transport between these zones is then not required in order to accurately obtain the surface abundance solution. To verify the accuracy of this assertion a model was calculated with homogenized abundances between all surface convection zones, and it was found that the surface solution was practically identical to the surface solution obtained when separation was allowed between SCZs. After a mere $3 \mathrm{Myr}$ of a star's main-sequence life with a mass loss rate of $10^{-13} M_{\odot} \mathrm{yr}^{-1}$, anomalies which appear at the surface reflect the separation which occurs below $\log \Delta M / M_{*}=-7$. Hence, for most of the main-sequence lifetime of models compatible with observations, the surface abundance solution depends on the separation which takes place around $\log \Delta M / M_{*} \simeq-5.5$ to -6.5 .

This is analogous to the turbulence models of Richer et al. (2000), in which surface abundances depend solely on the separation which occurs below $200000 \mathrm{~K}$. In our calculations, however, abundance variations are present throughout the stellar envelope because no mixing is enforced outside of convection zones. In that respect, in the presence of mass loss, it is clear that the chemical separation responsible for the AmFm phenomenon involves up to $\log \Delta M / M_{*}=-5$ of the star's mass.

\footnotetext{
13 Remember that at $t=0$, the flux distribution is essentially proportional to the local abundance multiplied by the local $g_{\text {rad }}$ since the composition is homogeneous.
} 


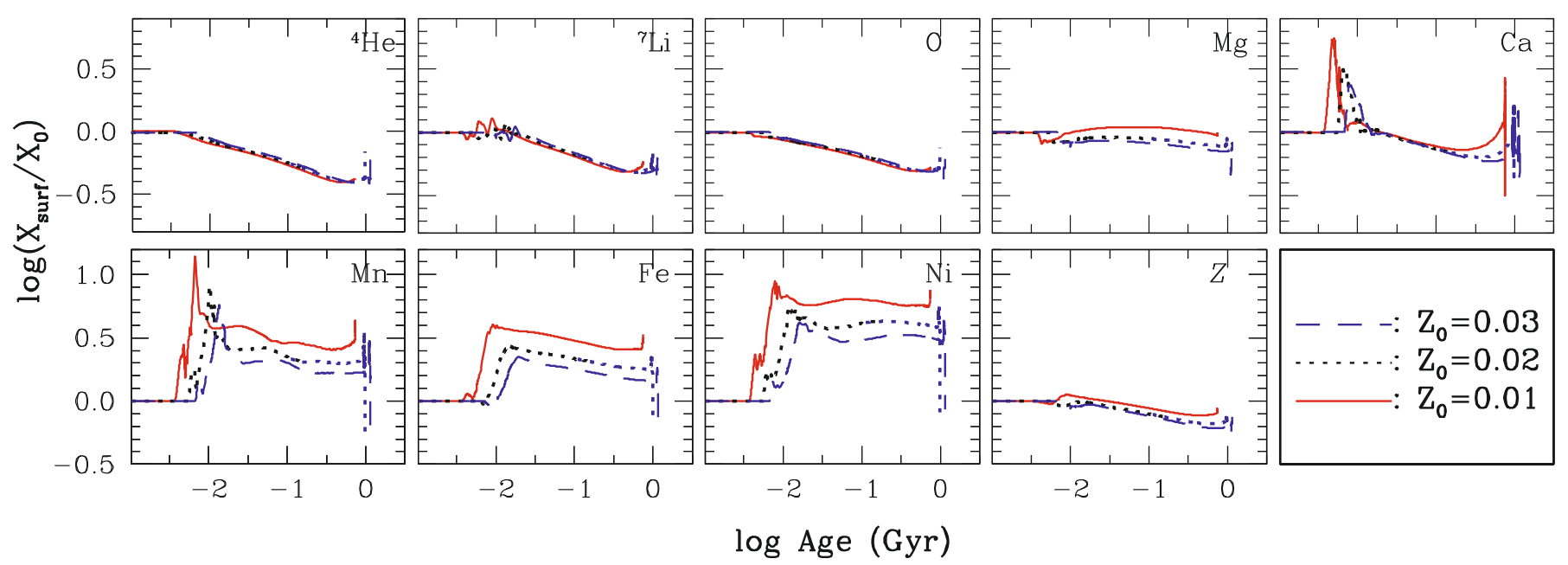

Fig. 13. The effect of varying initial metallicity on the evolution of abundance anomalies at the surface of a $2.0 M_{\odot}$ star with a mass loss rate of $10^{-13} M_{\odot} \mathrm{yr}^{-1}$. Models are shown for $Z_{0}=0.01$ (solid), $Z_{0}=0.02$ (dashed line) and $Z_{0}=0.03$ (long dashed line). The original abundance of Li is assumed independent of $Z_{0}$.

\subsubsection{The effect of $Z$, age and $T_{\text {eff }}$}

In Fig. 13, one sees that the main features of the time evolution of surface abundances are similar for three different initial metallicities. However varying the initial metallicity can have an important effect on the amplitude of surface anomalies, though not for all elements. For elements such as $\mathrm{CNO}$, varying $Z_{0}$ has relatively little effect on the surface abundance anomalies since these elements are not supported by the radiation field. For heavier iron peak elements, supported by the radiation field, the ramifications are much more apparent since their lines are often saturated and flux sharing becomes prevalent. In fact, the lower metallicity models have larger anomalies, when compared to the original abundances of the model, since the same flux is shared among fewer atoms. Also, the abundance peaks appear earlier during evolution as $Z$ decreases since the $g_{\text {rad }}$ profiles with respect to $\Delta M / M_{*}$ are shifted toward the surface as $Z$ decreases. However the situation is different if one compares to a fixed set of abundances. By comparing Figs. 11 and 13 one notes that a reduction of mass loss by a factor of 1.5 approximately compensates for a reduction of $Z_{0}$ from 0.03 to 0.02 for the absolute final abundance of $\mathrm{Fe}$ and other elements which are supported. It however amplifies the effect of the reduction for elements which are not supported by $g_{\text {rad }}$ such as CNO.

Figure 14 shows the surface abundances as a function of atomic number for a $2.5 M_{\odot}$ model at five different ages. A large overabundance of Ti (1.3 dex) occurs as early as 5 Myr because $g_{\mathrm{rad}}(\mathrm{Ti})$ has a maximum near the surface, and the bump it causes in the original flux is dragged by the wind. The same occurs for $\mathrm{Cr}$ and $\mathrm{Mn}$ but to a lesser extent. There is also a slight overabundance of $\mathrm{Li}$ which appears at this age. At $9 \mathrm{Myr}$, the Ti overabundance has weakened and most iron peak elements reach their highest values: Fe peaks at $0.65 \mathrm{dex}$ and $\mathrm{Ni}$ peaks at 1.0 dex. Throughout the star's main-sequence evolution, the surface abundances change only slightly, as highlighted by the similarity in the curves at 70 and $250 \mathrm{Myr}$. Until the final dredge-up (i.e. the post turn-off increase in SCZ mass), which begins around $520 \mathrm{Myr}$, the iron peak abundances decrease only slightly. For instance, Fe only drops 0.15 dex over the entire main-sequence lifetime. The changes will be slightly more significant for underabundant elements because they are not supported by the radiation field, and so the amplitude of

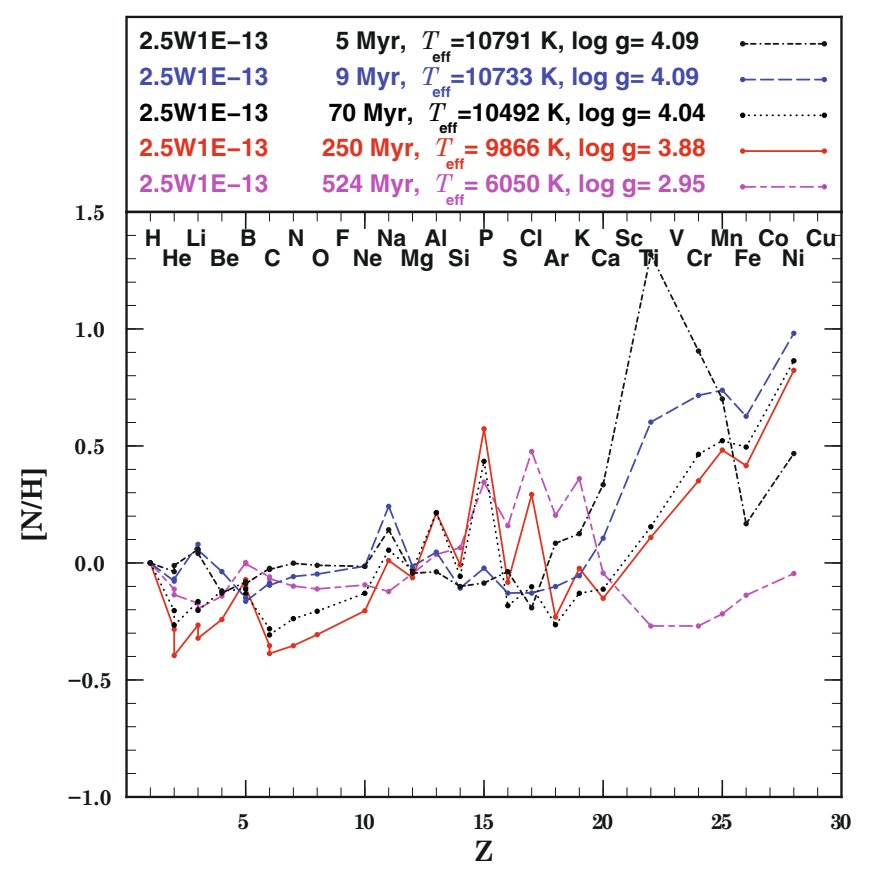

Fig. 14. Surface abundance anomalies at five ages $(5,9,70,250$ and $524 \mathrm{Myr}$ ) for a $2.50 M_{\odot}$ star with a mass loss rate of $10^{-13} M_{\odot} \mathrm{yr}^{-1}$.

the underabundances is largely dependant on time (due to settling). For CNO, the surface underabundances appear around $9 \mathrm{Myr}$ and continuously decrease down to about $-0.40,-0.38$ and -0.35 dex for $\mathrm{C}, \mathrm{N}$ and $\mathrm{O}$ respectively, after which dredgeup begins. At all times, the surface abundances reflect the matter which is exposed by the wind at the specific age. With that in mind, one notes from the curve at $524 \mathrm{Myr}$, that the material at $\log \Delta M / M_{*} \simeq-2$ has not yet reached the surface since LiBeB abundances have not yet dipped due to material advecting to the surface from regions where they burn (see Fig. 6). At this age, the surface convection zone extends down to $\log \Delta M / M_{*}=-4.5$, and while iron peak elements are now underabundant, an overabundance has appeared for $S$ (and a few species of similar atomic mass) because of the dredge up of the maximum of its abundance at $\log \Delta M / M_{*} \sim-5.2$ (see Fig. 6, one also notes the 


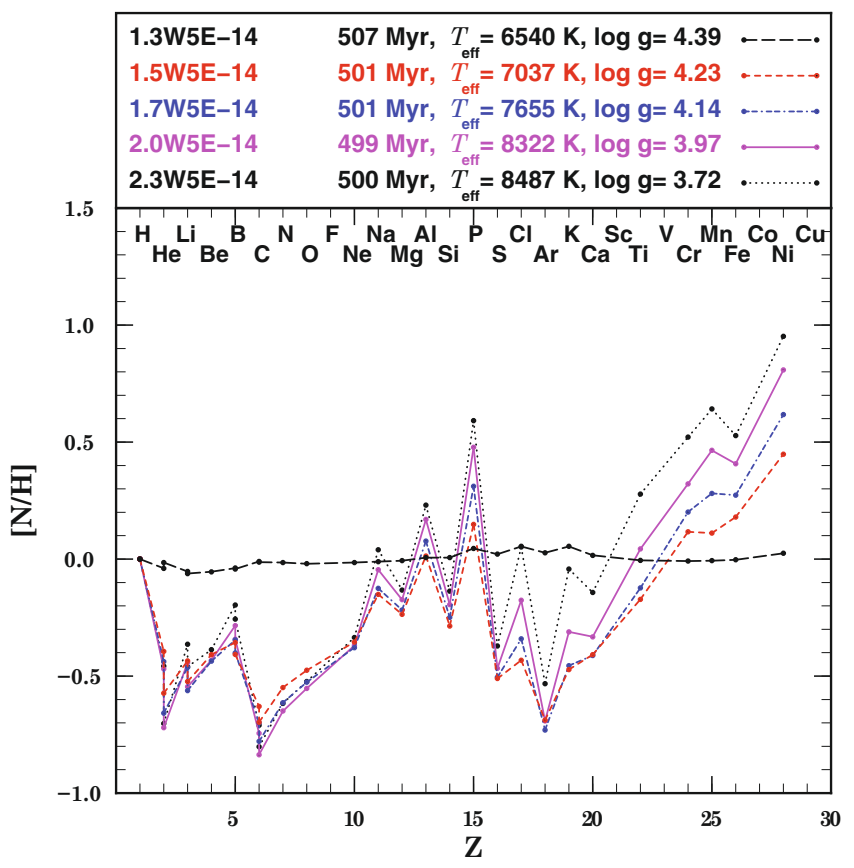

Fig. 15. The effect of varying $T_{\text {eff }}$ (or stellar mass) on the surface abundance profiles of 5 models at $500 \mathrm{Myr}$ for a mass loss rate of $5 \times 10^{-14} M_{\odot} \mathrm{yr}^{-1}$.

underabundances of $\mathrm{Fe}$ and $\mathrm{Ni}$ near $\log \Delta M / M_{*} \sim-5$ which explain their underabundance at $524 \mathrm{Myr}$ ). This time dependent behavior is compared to models with turbulence in Sect. 6 .

Figure 15 shows the superficial abundances at $500 \mathrm{Myr}$ for models of different masses with the same mass loss rate $\left(5 \times 10^{-14} M_{\odot} \mathrm{yr}^{-1}\right)$. The $1.3 M_{\odot}$ has much smaller anomalies than the others. At a given age, stars of 1.5 to $2.3 M_{\odot}$ have very similar underabundances of elements from $\mathrm{He}$ to $\mathrm{Ne}$, since for a given mass loss rate, they essentially depend on time. Iron peak overabundances increase as $T_{\text {eff }}$ increases. This is partly caused by the increase of $g_{\mathrm{rad}}$ with $T_{\text {eff }}$ as well as by the reduction of the mass of the surface convection (if the SCZ is more massive, the anomalies will be reduced by dilution). This behavior is different from what is obtained in turbulence models, and will also be discussed in Sect. 6.

\subsubsection{Stars of the lithium gap}

The surface Li abundances for stars of the lithium gap without mass loss are shown in Fig. 16. The models heavier than $1.45 M_{\odot}$ ceased converging before the end of their main-sequence lifetime because of numerical instabilities. In the absence of mass loss, the $\mathrm{H}-\mathrm{He}$ and iron peak convection zones are split by the appearance of a thin radiative zone for all models of $1.47 M_{\odot}$ or more. Variations within this layer appear at the surface of the $1.47 M_{\odot}$ model near $200 \mathrm{Myr}$.

A close inspection of Fig. 16 shows a disctinct separation between the $1.46 M_{\odot}$ and $1.47 M_{\odot}$ models; the $1.46 M_{\odot}$ model behaves more like the $1.45 M_{\odot}$ model while the $1.47 M_{\odot}$ model's behaviour most resembles the $1.48 M_{\odot}$ model. Again, this is because of the appearance of a radiative zone immediately above the iron peak $\mathrm{CZ}$ in the 2 heavier models.

The abundances obtained near the age of the Hyades open cluster ranged from -0.25 dex for the $1.37 M_{\odot}$ model to -1.25 dex for the $1.46 M_{\odot}$ model. Lithium is underabundant for all models throughout evolution.

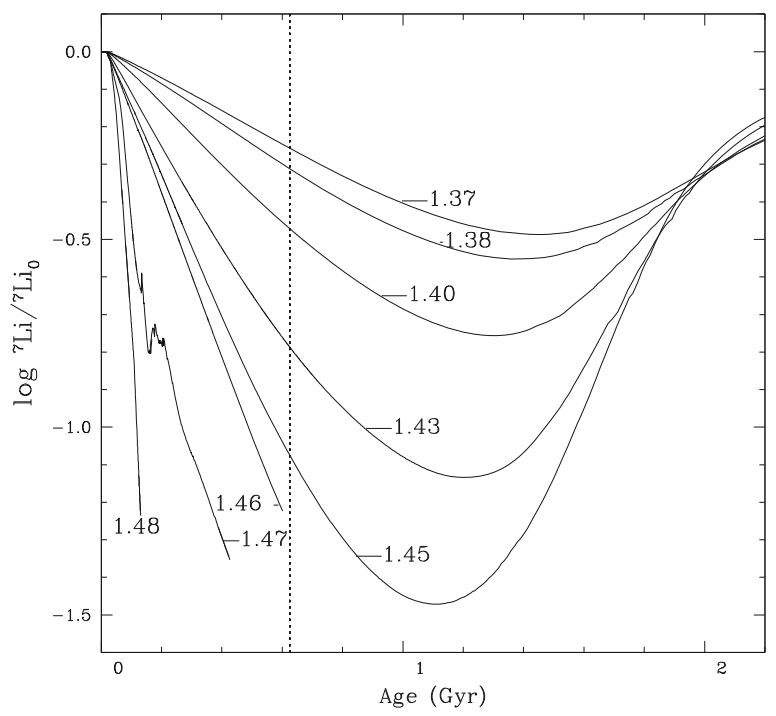

Fig. 16. Evolution of ${ }^{7} \mathrm{Li}$ surface abundances for models with masses ranging from $1.37 M_{\odot}$ to $1.48 M_{\odot}$ with no mass loss and $Z_{0}=0.02$. The vertical line indicates $625 \mathrm{Myr}$, the age of the Hyades open cluster. This can be compared to Fig. 6 of Richer \& Michaud (1993).

By comparing these results with those shown in Fig. 6 of Richer \& Michaud (1993), one first notices that the curves have very similar behavior in time; the minima occur at nearly the same age and the curve shapes are nearly identical. However, the underabundances obtained in our calculations are systematically smaller. For instance, their $1.43 M_{\odot}$ model is 250 times underabundant around $1.23 \mathrm{Gyr}$, while our $1.43 M_{\odot}$ model is only 14 times underabundant at the same age. A careful analysis of the results determined that the difference was principally due to the difference in the mass of the SCZ. If the convection zone is homogeneous and emptied through its bottom, then:

$\frac{\mathrm{d}\left(X \Delta M_{\mathrm{cz}}\right)}{\mathrm{d} t}=X w_{i} \rho$

where $X$ is the mass fraction in the SCZ (and on the surface), $\Delta M_{\mathrm{cz}}$ the mass in the SCZ, $w_{i}$ the diffusion velocity and $\rho$ the density immediately below the SCZ. By integrating this equation and comparing diffusion time scales for the different values of $M_{\mathrm{cz}}$, the differences in surface lithium abundances for models of the same mass can be explained by the difference in $M_{\mathrm{cz}}$. This difference in CZ mass is mainly due to the inclusion of metal diffusion in our models. In fact, the increased opacity in our models due to heavy metal accumulation considerably increases opacity at the BSCZ, thereby extending it inwards.

In Fig. 17, the surface lithium abundances are shown for different isochrones of the models shown in Fig. 16. The gap position evolves toward cooler temperatures with time. At $100 \mathrm{Myr}$, only stars of $6900 \mathrm{~K}$ have significant underabundances, while at $625 \mathrm{Myr}$, stars as cool as $6700 \mathrm{~K}$ have important underabundances.

At 100, 200 and $400 \mathrm{Myr}$, the gap obtained in the present calculations is $50-80 \mathrm{~K}$ hotter than the gap of the same metallicity shown in Fig. 8 of Richer \& Michaud (1993). Our gap is also slightly deeper, as the surface lithium on the hotter end of our isochrones keeps decreasing where surface lithium in the isochrones of Richer \& Michaud (1993) start increasing. This is a result of the smaller $g_{\mathrm{rad}}(\mathrm{Li})$ obtained in our models (see Sect. 3.1.2, in particular Fig. 3): the competition with $\mathrm{Fe}$ decreases $g_{\text {rad }}(\mathrm{Li})$ by a factor of 2 , so that it doesn't reach $g$ in 


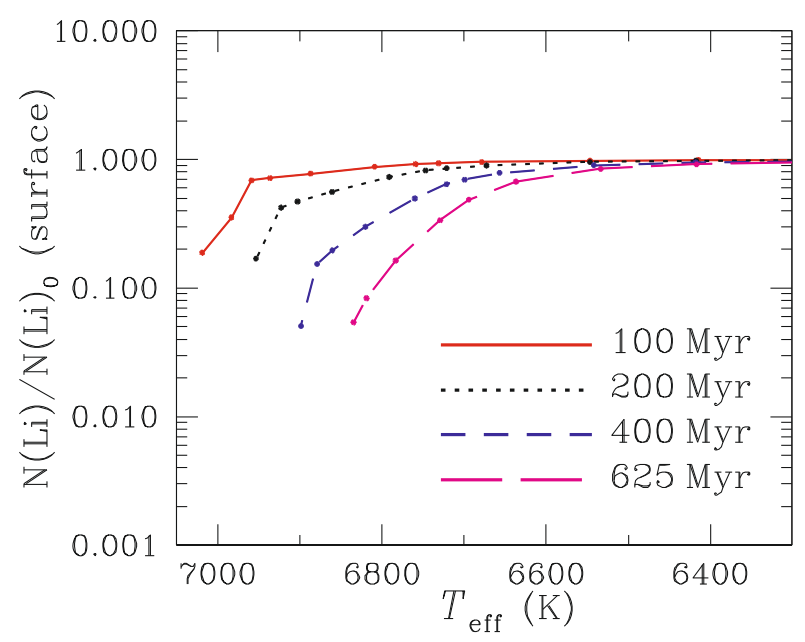

Fig. 17. The $T_{\text {eff- }} X_{\mathrm{Li}}$ relation at different ages for the models shown in Fig. 16. Each point represents a calculated model. This plot can be compared directly to Fig. 8 of Richer \& Michaud (1993).

the hotter models of our isochrones. Therefore, lithium is not supported in our diffusion only model.

\subsubsection{Separated winds}

The effects of various separated wind configurations on surface abundances of $1.4 M_{\odot}$ models are illustrated in Fig. 18. Along with the $1.40 \mathrm{~W} 1 \mathrm{E}-14$ unseparated mass loss model and the pure diffusion model (no wind), three cases of separated winds were considered, as described in Sect. 4.3.

In constrast to unseparated mass loss (dotted line) which, when comparing to the pure diffusion model (solid line) in Fig. 18, reduces surface anomalies for all elements, separated mass loss can affect underabundant and overabundant elements differently. In case 1 , for which all metals are ejected with the same relative concentration as in the atmosphere whereas $\mathrm{H}$ and He remain bound, a mass loss rate of $1 \times 10^{-16} M_{\odot} \mathrm{yr}^{-114}$ leads to smaller overabundances and larger underabundances than unseparated mass loss. This is because in case 1, the internal wind term this mass loss leads to is negligeable (see Eq. (6)). Therefore, for underabundant elements, the surface convection zone is drained from its bottom (because the wind is too weak to support downward diffusing elements), as well as from its top (i.e. mass loss at the stellar surface). Overabundant elements, on the other hand, are depleted through the surface, while the bottom of the surface convection zone is replenished by atomic diffusion. When the mass loss rate is increased to $5 \times 10^{-16} M_{\odot} \mathrm{yr}^{-1}$ for case 1 , the overabundances can rapidly evolve into underabundances since, without the wind providing sufficient replenishement from deeper inside the star, the elemental depletion at the surface quickly dominates the replenishement at the bottom of the convection zone. For the model with a mass loss rate of $5 \times 10^{-16} M_{\odot} \mathrm{yr}^{-1}$ underabundances of $\mathrm{Li}$ and $\mathrm{Fe}$ reach -1.5 dex and -0.95 dex respectively at $570 \mathrm{Myr}$, whereas for the $10^{-16} M_{\odot} \mathrm{yr}^{-1}$, the Li underabundance reaches -0.55 dex around $625 \mathrm{Myr}$, while Fe has an overabundane of 0.45 dex. Generalized underabundances are attainable in the context of case 1.

In case 2, all low-FIP elements are depleted 4 times more rapidly than $\mathrm{H}$ and other high-FIP elements. Consequently,

\footnotetext{
14 This is smaller than the rate of metal depletion for the unseparated model 1.40W1E-14, since $Z \times \dot{M}=1.999 \times 10^{-16} M_{\odot} \mathrm{yr}^{-1}$.
}

in comparison to the anomalies obtained for the $1.40 \mathrm{~W} 1 \mathrm{E}-14$ model, the anomalies for the low-FIP elements (of which Li, $\mathrm{Mg}, \mathrm{Ca}, \mathrm{Mn}, \mathrm{Fe}$ and $\mathrm{Ni}$ are shown) are greater for underabunt elements and smaller for overabundant elements, as in case 1. The Fe overabundance is reduced to $0.2 \mathrm{dex}$ around $625 \mathrm{Myr}$, and the $\mathrm{Li}$ underabundance reaches $-0.6 \mathrm{dex}$ at the same age. However, for $\mathrm{O}$, which is a high-FIP element, the curve is tucked in between the pure diffusion and unseparated mass loss curves (and is therefore impossible to see on the figure), which is what could be expected, since the rate of depletion as seen by $\mathrm{O}$, a high-FIP element, is smaller than $10^{-14} M_{\odot} \mathrm{yr}^{-1}$ because the lost mass has a higher concentration of low-FIP elements (see Sect. 4.3).

Finally, in case 3 , for which $\mathrm{H}$ is included among the lowFIP elements, the results are quite different: the depletion of $\mathrm{H}$ at the surface leads to an important increase of He. Since the $\mathrm{He}$ abundance at the surface remains slightly above its original value throughout the simulation, the stellar structure is changed as the $\mathrm{H}-\mathrm{He} \mathrm{SCZ}$ is much deeper on the main-sequence than for any of the other models. Around $625 \mathrm{Myr}, \mathrm{Fe}$ is barely underabundant, while $\mathrm{Li}$ is underabundant by $-0.5 \mathrm{dex}^{15}$. At $625 \mathrm{Myr}$, the model in case 3 is $100 \mathrm{~K}$ hotter than the three other models at the same age.

\section{Comparison to turbulence models}

As previously mentioned, models with turbulence (Richer et al. 2000; Richard et al. 2001; Michaud et al. 2005) have been quite successful at reproducing observed properties of AmFm stars both on the surface (i.e. abundance anomalies) and in the interior (pulsation properties of $\delta$ Scuti stars, see Turcotte et al. 2000). These models with turbulence put forth a scenario in which chemical separation takes place below $200000 \mathrm{~K}$, as opposed to the scenario in which chemical separation immediately below the $\mathrm{H}$ convection zone is responsible for AmFm abundance anomalies (Watson 1971; Alecian 1996). The models calculated with mass loss offer an alternative scenario which is in fact a hybrid of both these scenarios. On the one hand, no external mixing is enforced outside of convection zones, which allows for chemical separation to occur throughout the stellar interior. However, for most of the main-sequence lifetime, the surface abundance is modulated by matter which is advected from deep inside the star (while in turbulence models, it is mixed to that depth). In order to determine which of these scenarios must be favored, it is paramount to differentiate the models so as to enable observational tests.

Even when surface abundances are quite similar in turbulent and mass loss models, the interior behaves differently. This can be seen by comparing the 2.50W1E-13 and 2.50T5.2D1M-4 models in Figs. 19 and 20. In Fig. 19, which compares the internal concentrations of all 28 elements for a model with turbulent mixing and a model with mass loss, there is a stark contrast in the internal distribution of elements heavier than Ar, even though the surface abundances are quite similar for most atomic species (see Fig. 20). The Fe abundance in the interval $\log \Delta M / M_{*} \simeq-6$ to -5 is $4-5$ times larger in the turbulence model. The same can be said for Ni which, between $\log \Delta M / M_{*}=-7$ and -5 , differs by about a factor of 10 . This has an important effect on the local Rosseland opacity. These internal differences could therefore allow for some asteroseismological tests which could help differentiate between the two models.

\footnotetext{
15 For a given element, $[\mathrm{X} / \mathrm{H}]$ is about 0.1 dex $\operatorname{larger}$ than $\log \left(X_{\text {surf }} / X_{0}\right)$ because of the surface underabundance of $\mathrm{H}$.
} 

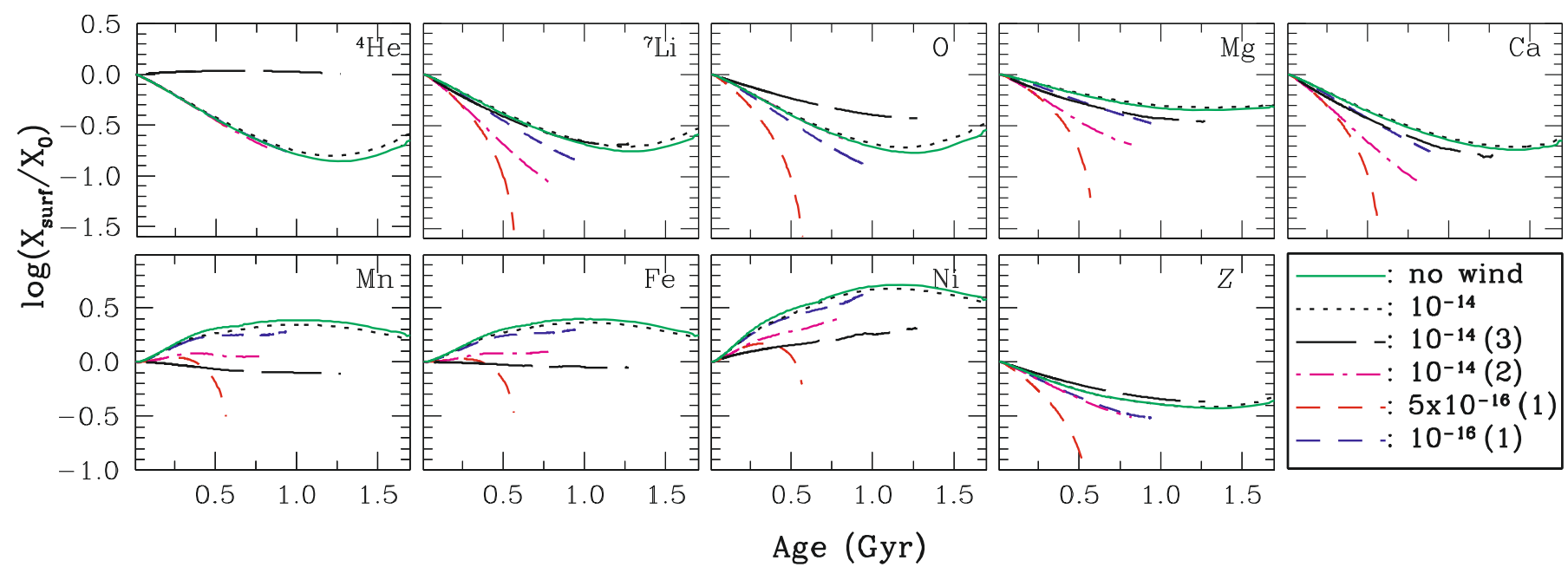

Fig. 18. Surface abundance evolution for selected elements of $1.40 M_{\odot}$ models with different types of wind solutions: one model with no mass loss (solid line), one model with a homogenous mass loss rate of $10^{-14} M_{\odot} \mathrm{yr}^{-1}$ (dotted line), and 4 models with separated mass loss. For the separated winds, the configuration is indicated by a number (1,2 or 3$)$ which is explained in Sect. 4.3 and in the text below.
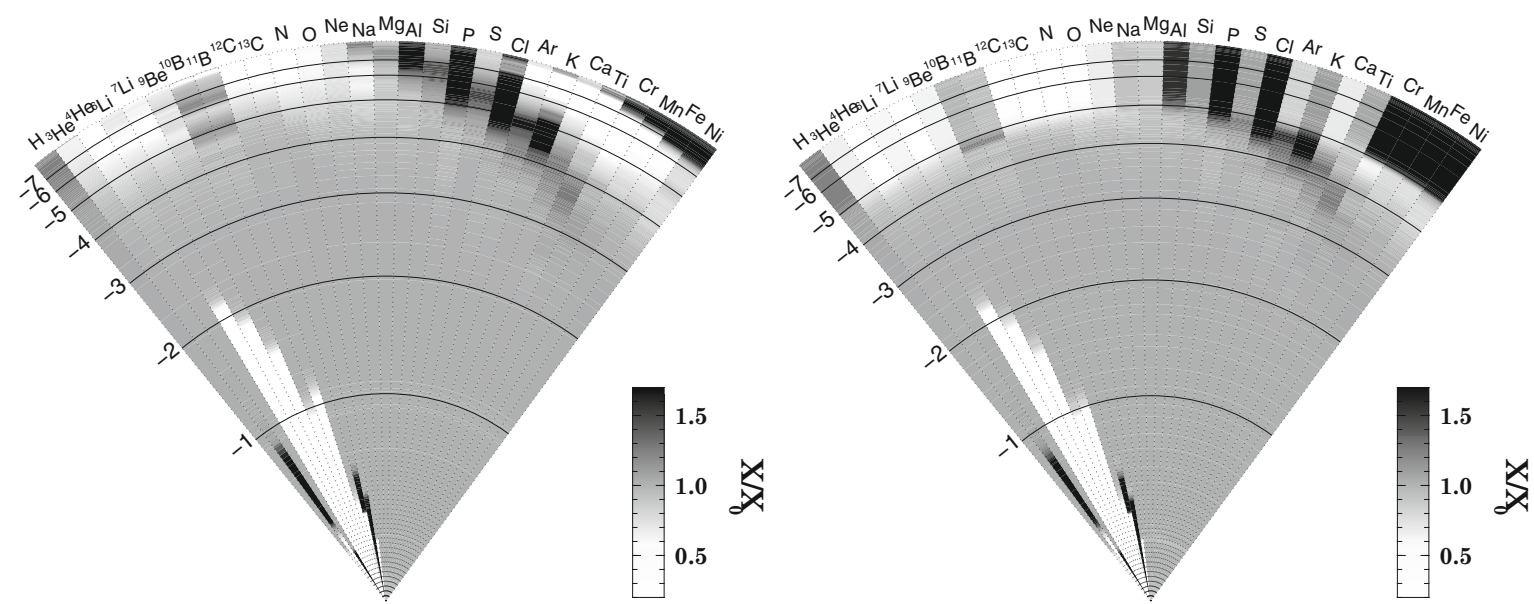

Fig. 19. Gray coded concentrations of two $2.50 M_{\odot}$ models at 500 Myr. The scale of the radius is linear although the value on the left hand side indicates $\log \Delta M / M_{*}$. The contrast is identified in the right insert and spans the interval from 0.3 to 1.7 times the original elemental abundances. The anomalies affect the outer $25 \%$ of the radius. Nuclear effects appear for lighter elements near the stellar core. For the calculations shown in the left panel, the competing process is mass loss while it is turbulence in the right panel. For these two models, the surface abundances are nearly the same (see Fig. 20).

With respect to pulsations, one notes that around $250 \mathrm{Myr}$, the age of Sirius (Liebert et al. 2005), the He II convection zone is still present in the turbulence model as well as in the $2.50 \mathrm{~W} 1 \mathrm{E}-13$ model, but not in the $2.50 \mathrm{~W} 5 \mathrm{E}-14$ model (see Fig. 9). As seen in the $\log \kappa_{R}$ panel, opacities are quite similar in the He II convection zones for the 2.50W1E-13 and the turbulence models, therefore, the mass loss model could be compatible with observed pulsation properties of $\delta$ Scuti stars (Turcotte et al. 2000). Further investigation is required to determine if the He II opacity bump in the $2.50 \mathrm{~W} 5 \mathrm{E}-14$ model is sufficient to drive kappa mechanism oscillations.

During evolution there are phases in which important surface abundance differences differentiate models with mass loss from those with turbulence. For instance, Fig. 14 can be compared directly to Fig. 15 of Richer et al. (2000). The most glaring difference is the surface behavior shortly after the onset of diffusion. In the turbulence model, abundance anomalies appear slowly and gradually at the surface, whereas large anomalies appear at the surface as early as $5 \mathrm{Myr}$ in the mass loss model. This is because all chemical separation that occurs near the surface, where timescales are short, will rapidly be advected to the surface by the wind ${ }^{16}$ (see discussion in Sect. 5.3.2). In the turbulence models, mixing is enforced throughout the upper envelope, and effectively prevents any chemical separation near the surface. Likewise, the abundances immediately following the turnoff are also different; while overabundant iron peak elements such as Mn and Fe can become underabundant as the SCZ exposes regions in the envelope with important gradients (as is the case at $524 \mathrm{Myr}$ ), the internal variations in the turbulence context are much less important (as an example, compare the Fe underabundance around $\log \Delta M / M_{*} \simeq-5$ in our Fig. 6 and Fig. 4 of Richer et al. 2000), and so the variations at the surface will also be smaller. On the main-sequence however, the differences between the two models are relatively quite small.

Similarly, for the $T_{\text {eff }}$ dependence, Fig. 15 can be compared directly to Fig. 16 of Richer et al. (2000). The most glaring difference is the behaviour of iron peak elements. By comparing

16 This will be discussed in further detail in a forthcoming paper which will look into the effects of diffusion on the pre-main-sequence. 


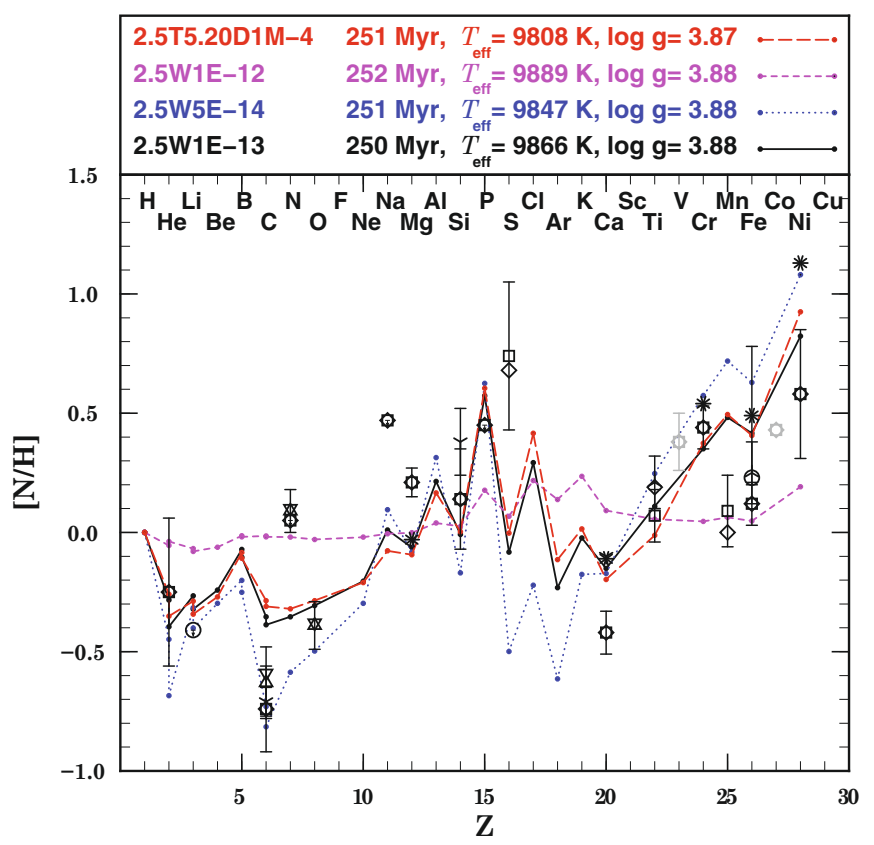

Fig. 20. Observations of the surface abundances of Sirius A (also known as $\alpha$ CMa, HR 2491 or HD 48915). Circles, Burkhart \& Coupry (1991); upright open triangles, Roby \& Lambert (1990); inverted open triangles, Lambert et al. (1982); three-point stars, Lemke (1989); inverted three-point stars, Lemke (1990); squares, Hill (1995); diamonds, Hill \& Landstreet (1993); asterisks, Hui-Bon-Hoa et al. (1997). Calculated values are shown for 3 models with mass loss (2.50W5E-14, 2.50W1E13 an 2.501E-12), as well as the model with turbulence (2.50T5.2D1M4) which represents the best fit from Fig. 18 of Richer et al. (2000). All models were calculated with a solar $(Z=0.02)$ initial metallicity and are shown at an age of 250 Myr. Vanadium, scandium and cobalt are grayed out since they are not included in our calculations. The internal abundance distributions of the 2.50W1E-13 and 2.50T5.2D1M-4 models are shown in Fig. 19.

the models with masses between 1.7 and $2.3 M_{\odot}$ in both figures, one notes that in the turbulence regime, the iron peak surface distribution is the same for all $T_{\text {eff }} \mathrm{S}$ at a given age, while there is more significant variations for the same elements in the mass loss models within the same stellar mass interval. On the contrary, the lighter elements show less variations in the mass loss models in comparison to the models with turbulence in the same mass interval.

\section{Comparison to observations}

In order to constrain stellar models, and to determine whether turbulence or mass loss is the dominant macroscopic process reducing surface abundance anomalies, it is imperative to compare our results with observations. In order to carry out an accurate comparison one needs to constrain age, mass and initial composition, which as shown in the previous section, all affect surface abundances. To reduce the arbitrariness of the comparison, we chose three open cluster stars for which we have a good evaluation of the initial metal content as well as of the approximate age. We will also compare our results to the field star Sirius A and the binary system $o$ Leonis.

In the following sections, $[\mathrm{N} / \mathrm{H}]$ has its usual meaning:

$[\mathrm{N} / \mathrm{H}]=\log (\mathrm{N} / \mathrm{H})_{\star}-\log (\mathrm{N} / \mathrm{H})_{\odot}$.

As mentioned in Sect. 2, this paper is part of a series of papers starting with Turcotte et al. (1998b), where the mixing length used was calibrated using the Sun for given boundary conditions, as well as helium and metal content. For consistency, the same boundary condition, solar composition and mixing length are used for Pop I stars. Our models lead to abundance variations, or anomalies, relative to those original abundances. A number of observers have similarly determined anomalies by differential methods with respect to solar abundances. Moreover, in the model atmospheres used for abundance determinations, most observers used the solar abundance mix either from Anders \& Grevesse (1989) or Grevesse et al. (1996). Because determinations were sometimes obtained with different solar photospheric abundances by different observers and sometimes with differential methods, their abundance determinations relative to the Sun (i.e. the anomalies) are used, when available, rather than absolute abundances. The uncertainty that inaccuracies in solar abundances lead to will be discussed in Sect. 8 .

\subsection{Field stars}

Sirius A is the most studied hot Am star $\left(T_{\text {eff }} \simeq 9800 \mathrm{~K}\right)$, with a mass of about $2.14 M_{\odot}$ and an age of approximately $250 \mathrm{Myr}$ (Gatewood \& Gatewood 1978; see also Sect. 4.1 of Richer et al. 2000). Figure 20 shows surface abundance determinations for 19 chemical species (16 of which are included in our calculations) from 8 different papers.

First, for most elements, there is considerable scatter among observers. For instance, there is a 0.3 dex difference in $\mathrm{Si}$ abundance, as well as a 0.4 dex difference in Fe abundance, which is the most carefully determined element.

We compared this data to 3 models with mass loss (2.50W1E-12, 2.50W1E-13 and 2.50W5E-14) as well as to the model with turbulence from Richer et al. (2000) which best reproduced the data $(2.50 \mathrm{~T} 5.2 \mathrm{D} 1 \mathrm{M}-4)$. Of the 16 observed elements which are included in our calculations, 12 ( $\mathrm{He}, \mathrm{Li}, \mathrm{O}, \mathrm{Na}$, $\mathrm{Mg}, \mathrm{Si}, \mathrm{P}, \mathrm{Ca}, \mathrm{Ti}, \mathrm{Cr}, \mathrm{Fe}$ and $\mathrm{Ni}$ ) are well reproduced by both the turbulence model and the $2.50 \mathrm{~W} 1 \mathrm{E}-13$ model. It is interesting to note that Bertin et al. (1995) determined, from $\mathrm{Mg}$ II lines, that the observed mass loss rate of Sirius A is between $1.5 \times 10^{-12}$ and $2 \times 10^{-13}$. The overall fit is also just as good for the $2.50 \mathrm{~W} 5 \mathrm{E}-14$ model since it is also able to reproduce the carbon abundance, though it perhaps overevaluates the iron peak abundances. The $\mathrm{S}$ abundance is not at all reproduced by our calculations; however, the observer gives little credibility to its value (Hill 1995). It is clear that the model with a mass loss rate of $10^{-12} M_{\odot} \mathrm{yr}^{-1}$ does not lead to the observed surface abundance pattern. Finally, surface abundance observations are not sufficiently accurate to enable a differentiation between turbulence and mass loss models.

In Michaud et al. (2005), the binary system $o$ Leonis (HD 83808/83809) has been interpreted as consisting of two AmFm stars with masses of 2.12 and $1.87 M_{\odot}$ (Griffin 2002). The authors show that two models with turbulent mixing, of 2.24 and $1.97 M_{\odot}$ respectively, are able to reproduce the observed features (namely both positions in the H-R diagram as well as surface abundances) of the A and B components of the binary system within the observational error bars. Similarly, models of 2.20 and $1.90 M_{\odot}$ with a mass loss rate of $5 \times 10^{-14} M_{\odot} \mathrm{yr}^{-1}$ are able to reproduce the $\mathrm{H}-\mathrm{R}$ position of both components. At the age indicated by the squares (750 Myr and $750.5 \mathrm{Myr})$, the A component is in the rapid evolution stage that follows the depletion of hydrogen in the core (the luminosity change is related to the star's adjustement to H-shell burning). At this age, the B component is still in the slowly evolving MS stage, which explains why both circles overlap on the graph. 


\section{$\log T_{\text {eft }}(\mathrm{K})$}

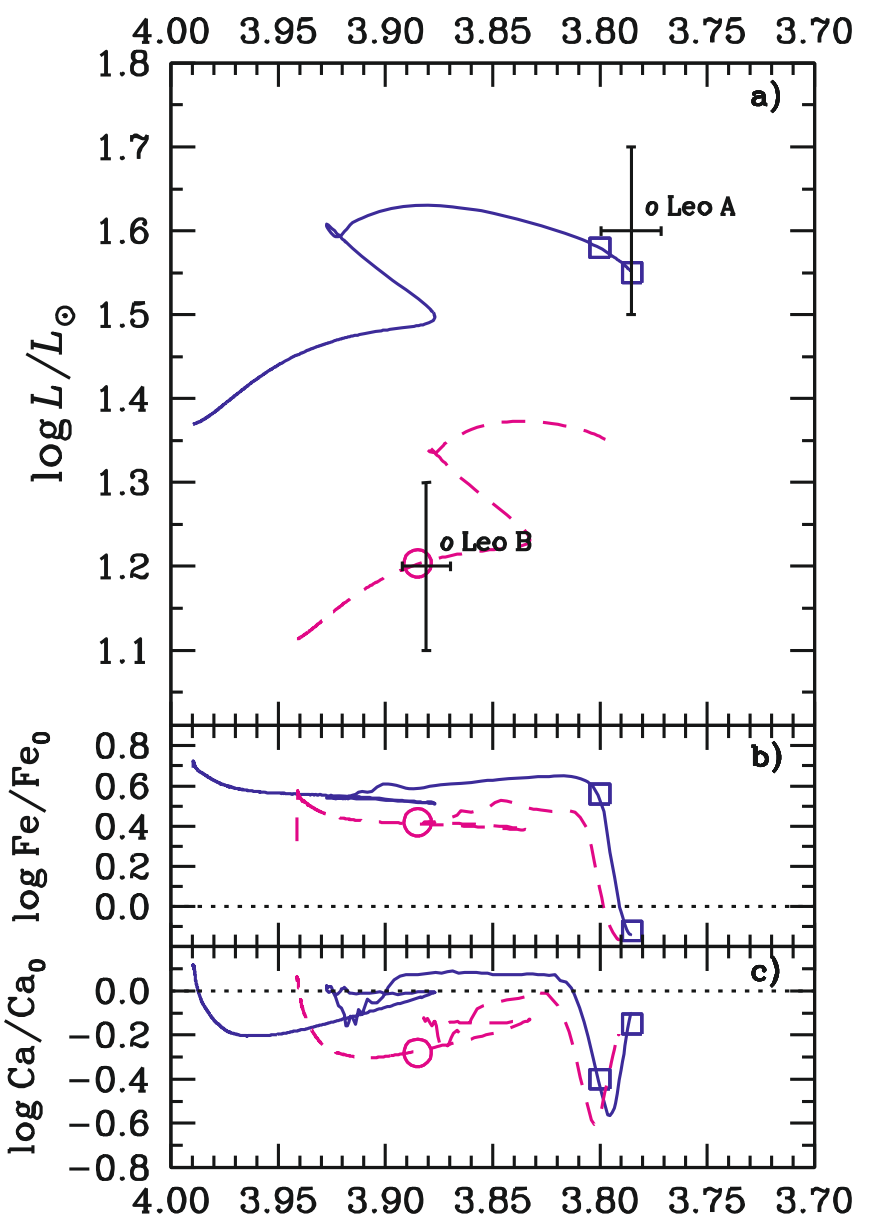

Fig. 21. A model of $2.2 M_{\odot}$ with a mass loss rate of $5 \times 10^{-14} M_{\odot} \mathrm{yr}^{-1}$ (solid line) and a model of $1.9 M_{\odot}$ with the same mass loss rate (dashed line) are plotted in an H-R diagram (a). The observed position in the $\mathrm{H}$ $\mathrm{R}$ diagram of the primary and secondary components of $o$ Leonis (with error bars) are shown with crosses. The squares indicate two possible positions on the primary's evolution path which fall within observational error bars. The circle indicates the position of the secondary at the age of the primary indicated by the square. The surface values of $\mathrm{Fe}$ and $\mathrm{Ca}$ are shown relative to their original values in (b) and (c).

The two squares in Fig. 21 show that the Fe abundance can vary between -0.2 dex and 0.6 dex within the $T_{\text {eff }}$ error bar for the A component, while the corresponding $\mathrm{Ca}$ surface abundance varies from -0.18 to -0.4 . Throughout most of this interval, the $2.20 M_{\odot}$ model has anomalies which are typical of Am stars (Fe overabundance coupled with a $\mathrm{Ca}$ underabundance). For either of the values for the A component, the $1.90 M_{\odot}$ model has an overabundance of $\mathrm{Fe}$ of 0.4 dex coupled with an underabundance of $\mathrm{Ca}$ which attains -0.3 dex, both typical Am star anomalies as well.

Both the turbulent model of Michaud et al. (2005) and the mass loss model can reproduce the AmFm character of components A and B. While the AmFm character of component A can be fitted by the turbulent model for its exact observed $T_{\text {eff }}$, the mass loss model only generates typical AmFm iron overabundances for a part of the error bar on the hot side of the observed $T_{\text {eff. }}$.

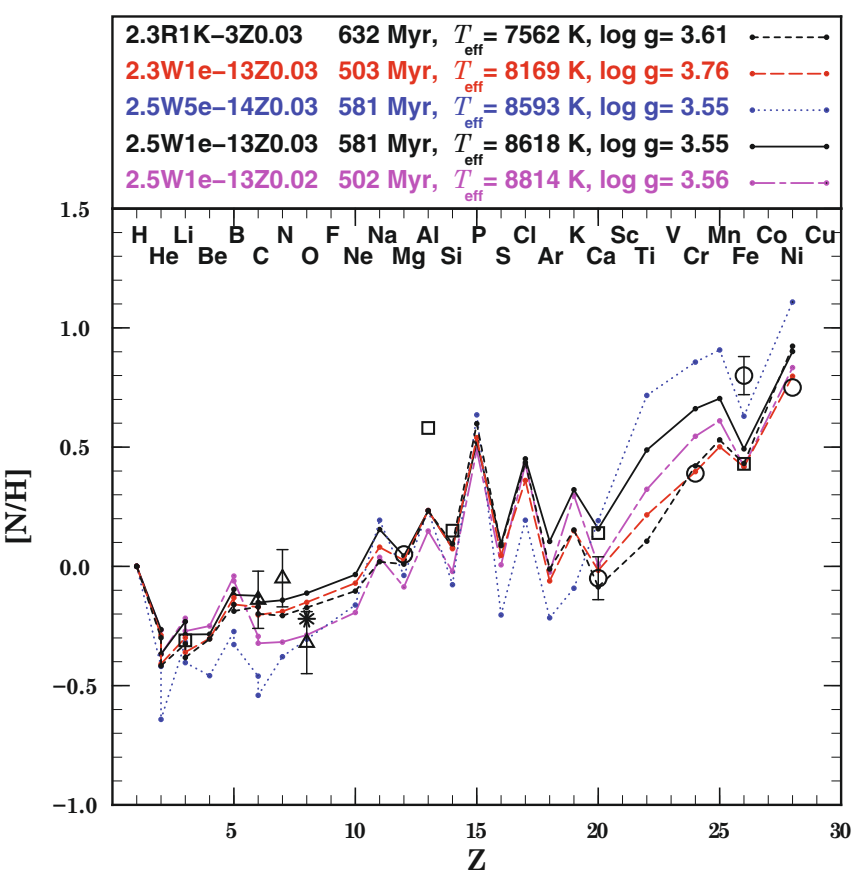

Fig. 22. Observed surface abundances of 68 Tau (also known as vB 56, HR 1389 or HD 27962), the hottest star (blue straggler) from the Hyades open cluster. Circles, Hui-Bon-Hoa \& Alecian (1998); triangles, Roby \& Lambert (1990); squares, Burkhart \& Coupry (1989); asterisks, Takeda \& Sadakane (1997). communication. Calculated values are shown for 4 models with varying mass loss rates as well as the model with turbulence which best reproduced the data $(2.30 \mathrm{R} 1 \mathrm{~K}-3 \mathrm{Z} 0.03$, see Richer et al. 2000). One model was calculated with $Z_{0}=0.02$, while all other models were calculated with an initial metallicity of $Z_{0}=0.03$. Metallicity is indicated in the model name.

\subsection{Open cluster stars}

In Fig. 22, we compare our results to observed abundance determinations for the hot Am star $68 \mathrm{Tau}\left(T_{\mathrm{eff}} \simeq 9050 \mathrm{~K}\right.$, Netopil et al. 2008) from the Hyades open cluster. The cluster age has been quoted between 625 Myr (Perryman et al. 1998) and 783 Myr by Varenne \& Monier (1999). Its quoted metallicity has also ranged between $Z_{0} \simeq 0.024$ (Perryman et al. 1998; Gratton 2000) ${ }^{17}$ and $Z_{0}=0.03$ (Cayrel et al. 1985) using $F$ and $\mathrm{G}$ star iron abundances as indicators. In order to reflect this metallicity, the selected models have been calculated with an initial metallicity of $Z_{0}=0.03$, which was also used in models from Richer et al. $(2000)^{18}$. We have attempted to make a compromise between fitting age and $T_{\text {eff }}$ : three models have a mass of $2.50 M_{\odot}$ and one has a mass of $2.30 M_{\odot}$. The $2.50 \mathrm{~W} 1 \mathrm{E}-$ $13 Z 0.03$ and $2.50 \mathrm{~W} 5 \mathrm{E}-14 \mathrm{Z} 0.03$ models are on a short $T_{\text {eff }}$ upswing which arises as hydrogen nears depletion in the core (in Fig. 21 for instance, it is the segment which immediately follows the main-sequence, spanning from $\log T_{\text {eff }} \simeq 3.87$ at its bottom to $\log T_{\text {eff }} \simeq 3.93$ at its top). In terms of stellar age, this upswing only lasts $3 \mathrm{Myr}$ before the star starts its descent onto the red giant branch. While it has very little effect on surface abundances, models were chosen at this age in order to be

${ }_{17}$ Using the Hyades iron enrichment factor of Gratton (2000) to multiply the metallicity determined by Asplund et al. (2009) for the solar mixture, the Hyades metallicity becomes $Z_{0} \simeq 0.019$. The actual value would likely lie somewhere between 0.019 and 0.03 . The curve with $Z_{0}=0.02$ in Fig. 22 illustrates the impact of uncertainties.

${ }^{18}$ Of course, $[\mathrm{N} / \mathrm{H}]$ at $t=0$ for models with $Z_{0}=0.03$ is above solar for all metals. 


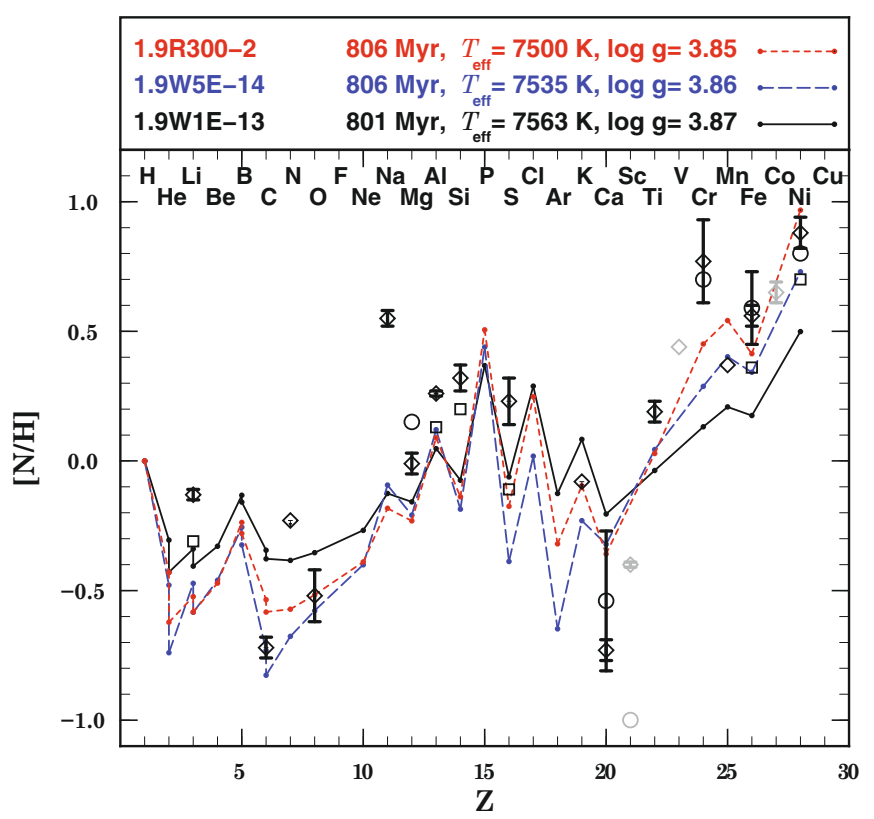

Fig. 23. Observed surface abundances of HD 73045 ( $T_{\text {eff }} \simeq 7500 \mathrm{~K}$ ) of the Praesepe open cluster which has an approximate age of $800 \mathrm{Myr}$. Circles, Hui-Bon-Hoa \& Alecian (1998); squares, Burkhart \& Coupry (2000); diamonds, Fossati et al. (2007). Curves correspond to models listed at the top of the figure. All models were computed with solar initial abundances $\left(Z_{0}=0.02\right)$.

closer to the star's surface temperature. Given the large spread in abundances between observers, the fit is almost perfect with the 2.30W1E-13Z0.03 model, which is slightly cooler and younger, yet is still on the main-sequence. Of 15 observed elements, only $\mathrm{Na}, \mathrm{Al}$ and $\mathrm{Mn}$ (arguably just $\mathrm{Al}$ ) are not reproduced. The fit is as good if not better (because of $\mathrm{Ni}$ ) than the fit obtained with the model with turbulence. The 2.50W1e-13Z0.02 model was added in order to illustrate the effect of reducing initial metallicity on absolute abundances (see also Fig. 13). The fit with observations is better than for the 2.50W1-13Z0.03 model, since the iron peak abundances with respect to solar abundances are smaller in the lower metallicity model. By comparing these two curves one can conclude that, for a given mass loss rate, a 0.18 dex reduction of initial metallicity can, at most, lead to a 0.18 dex reduction for elements which are not supported, such as $\mathrm{C}, \mathrm{N}$ and $\mathrm{O}$, and a 0.09 dex reduction for elements which are supported such as Fe. See also Sect. 5.3.3.

In Fig. 23, we compare 2 models of $1.9 M_{\odot}$ with mass loss as well as a model with turbulence to the observations of the star HD 73045 ( $T_{\text {eff }} \simeq 7500 \mathrm{~K}$ ) from the Praesepe open cluster which has an approximate age of $800 \mathrm{Myr}$ and a solar metallicity. There are 15 observed elements which can be compared to our simulations, although 3 determinations ( $N, \mathrm{~K}$ and $\mathrm{Mn}$ ), result from a single line and therefore could be inacurrate. Again, note the large discrepancies between observers. Only the 1.90W5E14 and the turbulence model can reproduce either the overabundant iron peak elements or the underabundances of $\mathrm{C}$ and $\mathrm{O}$. The abundances of $\mathrm{Na}$ and $\mathrm{Si}$ are not reproduced by either of the models.

Finally, we have compared our models to observations of the Coma Berenices star HD 108486 (Fig. 24). Coma Berenices is an open cluster with an age of about $500 \mathrm{Myr}$ and with a metallicity which is about solar. We have matched the star's $T_{\text {eff }}$ and age quite well with two $1.8 M_{\odot}$ models with mass loss. Except for $\mathrm{O}$ and $\mathrm{Na}$, which are not reproduced by any of the two models,

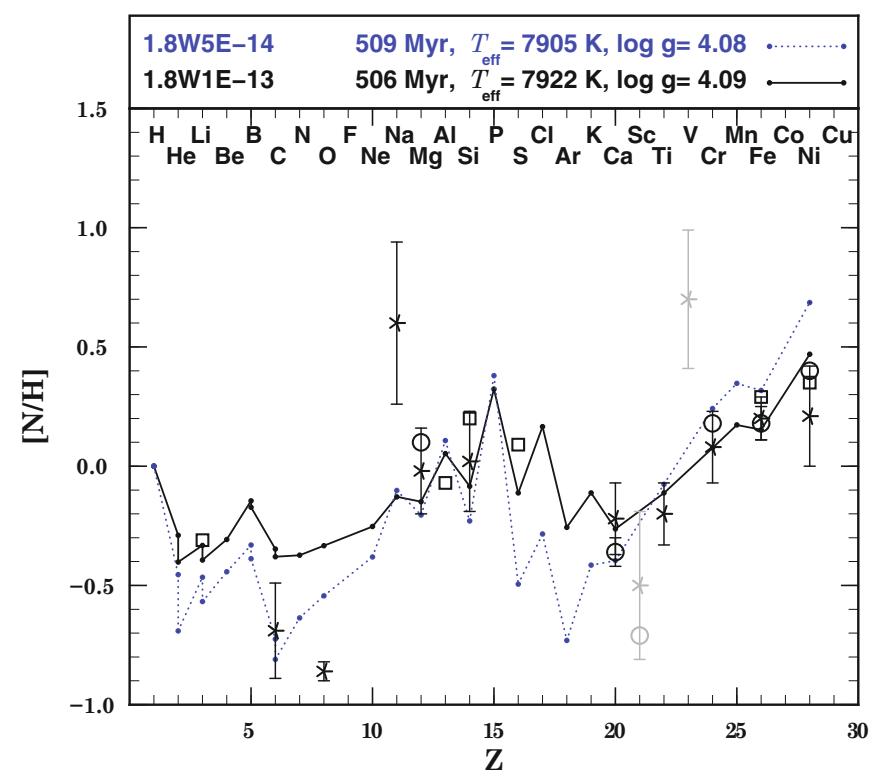

Fig. 24. Observed surface abundances of HD $108486\left(T_{\mathrm{eff}} \simeq 8180 \mathrm{~K}\right)$ of the Coma open cluster which has an approximate age of $500 \mathrm{Myr}$. Circles, Hui-Bon-Hoa \& Alecian (1998); squares, Burkhart \& Coupry (2000); five-point stars, Gebran et al. (2008). Curves correspond to models listed at the top of the figure. All models were computed with solar initial abundances $\left(Z_{0}=0.02\right)$.

most elements are fitted by both models. Assuming error bars for $\mathrm{S}$ and $\mathrm{Al}$ which are similar to those for other elements, we can state that 10 of the 13 abundances can be reproduced by the $1.80 \mathrm{~W} 1 \mathrm{E}-13$ model, and 9 by the $1.80 \mathrm{~W} 5 \mathrm{E}-14$ model.

Although we have opted not to add any extra figures, our results are also compatible with observations of $\mathrm{Ca}$ overabundances (see Fig. 12) in very young open clusters such as the Pleiades (Gebran \& Monier 2008; Hui-Bon-Hoa \& Alecian 1998) and $\alpha$ Persei (Hui-Bon-Hoa 1999). This is noteworthy since models with turbulence do not predict such an overabundance (see Figs. 10 of Richer et al. 2000).

\subsubsection{Lithium gap}

In Fig. 25, models with and without mass loss are compared to lithium, beryllium, calcium and iron observations in and around the Hyades lithium gap. Lithium determinations are shown for F stars and AmFm stars (normal A stars and other peculiar A stars are omitted). Iron and calcium abundances are shown only for stars which had a lithium determination. All beryllium abundances for F stars in Boesgaard \& King (2002) are shown ${ }^{19}$. When multiple observations for the same star were available, the different determinations are connected by a line segment. This gives an evaluation of the uncertainty. All models calculated with mass loss rates of $5 \times 10^{-14} M_{\odot} \mathrm{yr}^{-1}$ and $1 \times 10^{-13} M_{\odot} \mathrm{yr}^{-1}$ which were still on the main-sequence at $625 \mathrm{Myr}$ are shown. Models with a mass loss rate of $1 \times 10^{-14} M_{\odot} \mathrm{yr}^{-1}$ are omitted since they result in surface abundances which are very similar to diffusion only models (compare the diffusion only and 1.40W1E-14 models in Fig. 18). Therefore in the following

19 Lithium abundances from Boesgaard \& Tripicco (1986) and Boesgaard \& Budge (1988) are prefered over the revised values from Boesgaard \& King (2002) because there is a greater number of stars. Nonetheless, in the more recent paper, all previous determinations are revised upwards by 0.09 to 0.4 dex. 


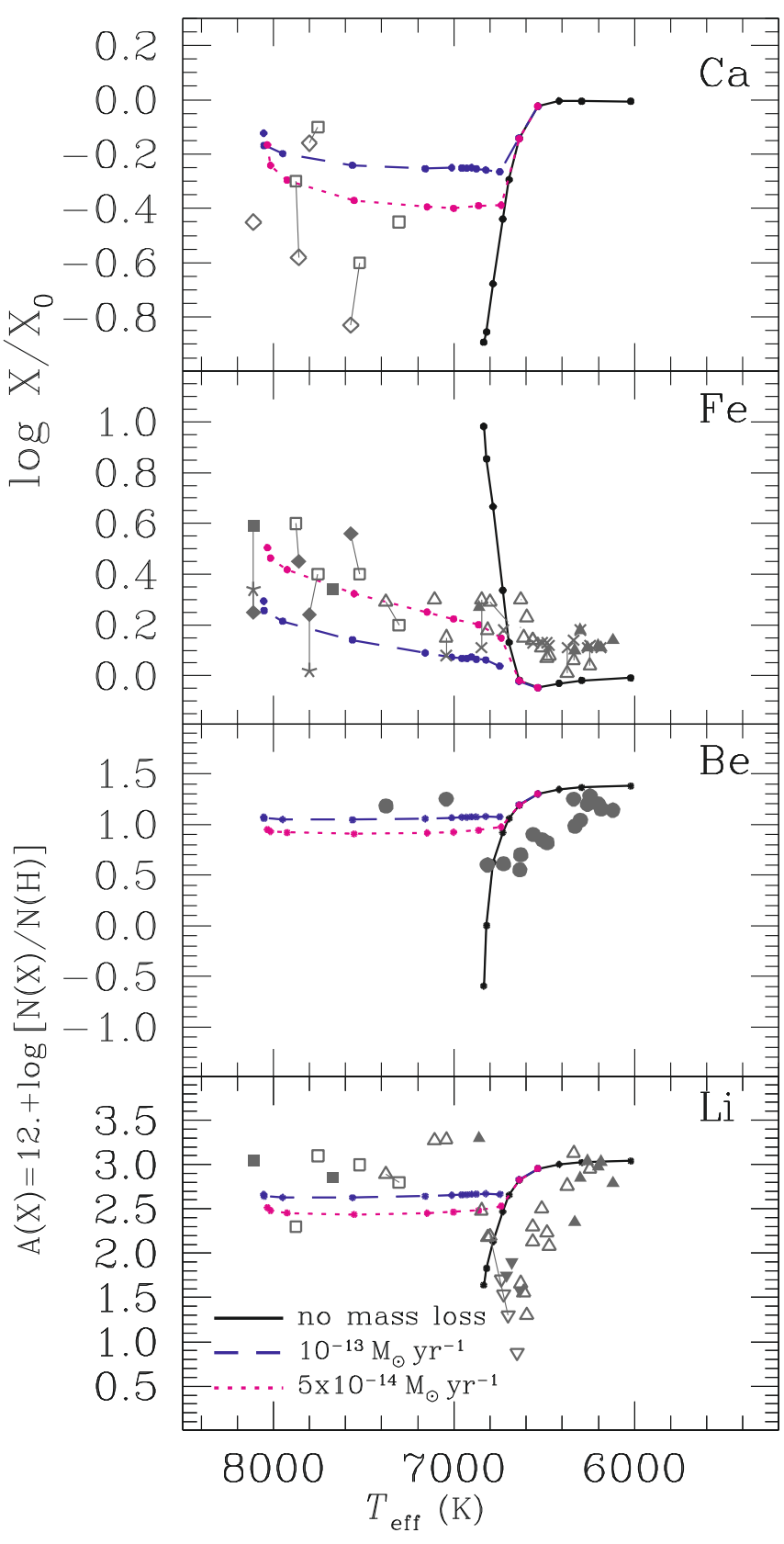

Fig. 25. Lithium, beryllium, iron and calcium abundances for models with and without mass loss at $625 \mathrm{Myr}$, the approximate age of the Hyades open cluster. All models were calculated with an initial metallicity of $Z_{0}=0.02$ and the original $\mathrm{Li}$ abundance was set at $\mathrm{N}(\mathrm{Li})=3.05$. The Li observations are from ( $\triangle$, and $\nabla$ for upper-limits) Boesgaard \& Tripicco (1986), ( $\boldsymbol{\Delta}$ and $\mathbf{v}$ ) Boesgaard \& Budge (1988), ( $\square$ ) Burkhart \& Coupry (1989) and (ם) Burkhart \& Coupry (2000). Be abundances are taken from $(\bullet)$ Boesgaard \& King (2002). Additional calcium and iron abundances are also shown for all stars with lithium determinations: (×) Boesgaard \& Friel (1990); ( $\star$ ) Takeda \& Sadakane (1997); ( $\diamond)$ HuiBon-Hoa \& Alecian (1998). All stars with multiple determinations are connected by a line segments. Calculated models are indicated by dots along the curves.

discussion, results from diffusion only models can be assimilated to models with unseparated mass loss $\leq 1 \times 10^{-14} M_{\odot} \mathrm{yr}^{-1}$. All models were calculated with an initial metallicity of $Z_{0}=0.02$, although the metallicity of the Hyades is above solar $(Z=$ 0.024 , see Sect. 7.2). The original value of lithium was set to $\mathrm{A}(\mathrm{Li})=3.05$. This value fits lithium determinations for the stars at the top of the cold side of the gap, which, since diffusion plays only a small role for these stars, probably reflect the cluster's original Li content (unless there is significant pre-main-sequence burning). Following the same logic, $\mathrm{A}(\mathrm{Be})$ was set to 1.40 .

According to our calculations, atomic diffusion in the absence of competing processes leads to an important reduction of surface lithium abundance. The smallest lithium anomaly was obtained for the $1.10 M_{\odot}$ model, for which surface lithium was reduced by $0.015 \mathrm{dex}$ at $625 \mathrm{Myr}$. The largest lithium reduction at $625 \mathrm{Myr}$ is by about $-1.4 \mathrm{dex}$ (or a factor of 25), for the $1.46 M_{\odot}$ model without mass loss. This does not quite reach the bottom of the gap of Boesgaard \& Tripicco $(1986)^{20}$. As seen in Fig. 16, the $1.47 M_{\odot}$ model without mass loss would likely have reached a lower lithium abundance had it been able to converge up to the age of the Hyades, thus reconciling some of the difference. In fact, the mass of the model which would have attained the lithium gap minimum can be deduced from Fig. 3 . Because Li is not supported until $g_{\text {rad }} \simeq g$ just below the surface convection zone at $625 \mathrm{Myr}$, the heaviest model for which the BSCZ is located where $\log T \gtrsim 5.4$ throughout its evolution will represent the gap minimum, since it is for this model that $\mathrm{Li}$ is sinking fastest. From Fig. 3, while the $1.55 M_{\odot}$ model is clearly on the hot side and the $1.43 M_{\odot}$ model on the cold side, it is the $1.46 M_{\odot}$ model that should be closest to the bottom of the gap. Furthermore, if the competition with Fe that is prescribed by the OPAL opacities and calculated in these models without mass loss is correct, then diffusion alone cannot explain the increase of $\mathrm{Li}$ on the hot side of the gap. The $g_{\mathrm{rad}}(\mathrm{Li})$ in the atomic diffusion only models will remain smaller than $g$ by a factor of at least 2 . However, given the uncertainties discussed in Sect. 3.1.1 on the location of Fe lines, $g_{\text {rad }}(\mathrm{Li})$ could very well attain $g$ near $\log T \simeq 5.3$, in which case lithium would be supported, and would consequently exhibit different surface behavior.

For the models without mass loss on the cold side of the gap, the Fe abundances are in agreement with observations up to about $6800 \mathrm{~K}$, after which the calculated overabundances become too large. The discrepancy between the Fe curve and the observations for $T_{\text {eff }}<6700 \mathrm{~K}$ is related to our models having a solar initial metallicity, whereas the Hyades stars were formed in a metal rich environment.

The models with mass loss rates of $5 \times 10^{-14} M_{\odot} \mathrm{yr}^{-1}$ and $10^{-13} M_{\odot} \mathrm{yr}^{-1}$ cannot explain the depletion encountered within the gap. However, given reasonable error bars, they are consistent with the almost constant lithium and beryllium abundances observed for $T_{\text {eff }}>7200 \mathrm{~K}$. Both mass loss rates lead to models which reproduce the observed Fe abundances between $6000 \leq T_{\text {eff }} \leq 8200 \mathrm{~K}$ and, in particular, the increase in Fe abundance for $T_{\text {eff }}>7200 \mathrm{~K}$, which is compatible with the AmFm character of these stars. Given the large discrepencies in determinations, most $\mathrm{Ca}$ abundances are also compatible with the models with mass loss.

Neither the calculated gap minimum nor the shoulder on the cold side of the gap match the observed position in $T_{\text {eff }}$. By comparison to Fig. 9 of Richer \& Michaud (1993), their calculated depth for the gap $(-1.6 \mathrm{dex})$ resembles the depth obtained in our calculations (-1.4 dex, see discussion in Sect. 5.3.4). The shoulder on the cold side of the gap obtained in the present calculations matches the curve they obtained with $Z_{0}=0.02$ within ${ }^{20}$ It has been suggested to revise these observations upwards by up to
0.4 dex (see the discussion in Sect. 2.1.2 of Michaud \& Charbonneau
1991 ; and Boesgaard \& King 2002). The maximum depletion encountered in the deepest part of the gap might so be closer to a factor of 50 . 
$\pm 50 \mathrm{~K}$. Accordingly, as also seen in this same Fig. 9, if we had chosen $Z_{0}=0.03([\mathrm{Fe} / \mathrm{H}]=+0.18)$, some of the $150 \mathrm{~K}$ difference would have been recuperated as the gap minimum would have been shifted toward cooler temperatures by $50-80 \mathrm{~K}$. There is also a $\pm 50-100 \mathrm{~K}$ uncertainty on the observed potition of the gap (see discussion in Sect. 2.1.2 of Michaud \& Charbonneau 1991). The uncertainty on the age of the Hyades (from 625 to $783 \mathrm{Myr}$, see Sect. 7.2) could also account for some of the difference as illustrated in Fig. 17. As the age of the isochrones increases, the $T_{\text {eff }}$ at which lithium abundances fall off also decreases. The real problem in explaining the Li gap with atomic diffusion is not with the exact $T_{\text {eff }}$ of the gap nor its depth, but rather with the calculated $\mathrm{Fe}$ overabundances which are not observed, and the related difficulty in calculating $g_{\mathrm{rad}}(\mathrm{Li})$ on the hot side of the gap due to Fe lines.

\section{General discussion and conclusion}

\subsection{Summary of results}

Evolutionary models including both atomic diffusion and unseparated mass loss explain the main abundance anomalies of AmFm stars (Sect. 7.2). When mass loss is assumed to be the only macroscopic process competing with atomic diffusion, observed abundance anomalies from open cluster stars as well as Sirius A and $o$ Leonis constrain mass loss rates to 2-5 times the solar mass loss rate. As shown in Sects.7.1 and 7.2, models involving mass loss are as capable as models involving turbulence in explaining observations of AmFm stars. This is because in both instances, the important separation occurs at the same depth $\left(\Delta M / M_{*} \simeq 10^{-6}-10^{-5}\right)$ for most of the main-sequence life. Whether the mass loss model is to be preferred over the turbulence model is difficult to assess given the large observational uncertainties. However, as shown in Fig. 19, the internal distribution of elements is different between the two cases for most elements. With differences reaching a factor of 4-5 for abundant elements such as Fe, there should be effects on local opacities and thus on pulsations. Asteroseismic tests could perhaps distinguish between the two ${ }^{21}$.

In the mass loss regime, chemical separation affects up to $10^{-5} M_{\odot}$ of a star's mass or, equivalently, 20 to $25 \%$ of the stellar radius (see Fig. 6 and Fig. 19). For any given element, as long as the wind velocity is greater in amplitude than the downward settling velocity, the local abundance solution is determined by flux conservation; local abundances adjust as the flux quickly becomes constant throughout the outer envelope (see Fig. 7). As a result, the surface abundances depend on matter which is advected from deep inside the star (see discussion in Sect. 5.3.2). This differs from the models of Watson (1971) and Alecian (1996) in which surface abundances depend on the outer $10^{-10} M_{\odot}$. This can also be contrasted to the solution obtained in the models with weak or fully separated mass loss presented in Sects. 5.2.1 and 5.3.5.

When flux is conserved throughout the envelope, abundance gradients which form near the surface, between surface convections zones for example, have no effect on the surface solution once the star has arrived on the main-sequence (see discussion in Sects. 5.3.2 and 5.3.3). In this instance, if the age of interest is greater than $M_{\mathrm{BSCZ}} / \dot{M}$, where $M_{\mathrm{BSCZ}}$ is the mass above the bottom of the deepest surface convection zone, one can obtain a nearly similar surface solution by approximating that abundances are homogeneous from the surface to the bottom of the

\footnotetext{
21 Carrier et al. (2007) did not detect pulsations which could have been a signature of iron accumulation in the Am star HD 209625.
}

deepest SCZ. However, early in the evolution, only matter from superficial layers has had time to be advected, and thus surface abundances obtained here depend on separation that occured close to the surface as first studied for Ca by Alecian (1996, see also Sect. 5.3.1) and confirmed observationally (see end of Sect. 7.2). This favors models involving mass loss rather than turbulence. Likewise, variations obtained near the surface, which do not appear in models with turbulence, have an effect on the PMS (Fig. 14) and will be discussed in a forthcoming paper.

In all models heavier than $1.47 M_{\odot}$ without mass loss or with an unseparated mass loss rate $\leq 1 \times 10^{-14} M_{\odot} \mathrm{yr}^{-1}$, the accumulation of $\mathrm{Fe}$ and $\mathrm{Ni}$ around $T=200000 \mathrm{~K}$ leads to the appearance of a thin radiative layer which separates the iron peak convection zone from the surface $\mathrm{H}-\mathrm{He}$ convection zone. This accumulation forms before the appearance of a small inversion of the local molecular weight gradient (see Sect. 5.2.1). The inclusion of thermohaline convection as suggested by Théado et al. (2009) could have an effect on abundances in the region, though convection occurs even when there is no molecular weight gradient inversion. This would require further investigation. Nonetheless, the appearance (or not) of the iron peak convection zone does not have a significant effect on the surface solution, nor does it appear in models with mass loss which adequately reproduce observed abundance anomalies of AmFm stars (Sect. 7).

Since this paper is a part of a series which explores the various macroscopic processes which compete with atomic diffusion in AmFm stars, it is important that the models be as similar as possible to those used in previous calculations (e.g. those with turbulence) in order to isolate the effects due specifically to mass loss. This is one of the primary motivations for using the same initial solar abundances as in previous calculations, rather than the revised Asplund et al. (2005, 2009) abundances (see also the discussion in Sect.2). Varying too many things at once could obscure results and introduce further uncertainty. Furthermore, there is a controversy on solar abundances, since heliosismology strongly favors the older (Grevesse et al. 1996) over the newer (Asplund et al. 2005, 2009) composition. One may then view the abundance differences between the two sets as an evaluation of uncertainty. Since solar abundances are used throughout this paper, the uncertainty on solar abundances leads to uncertainties on the absolute values of all abundances. As shown in Fig. 22, a factor of 1.5 reduction (or $0.18 \mathrm{dex}$ ) of the original $Z$ leads to a similar reduction $(0.18 \mathrm{dex})$ of the expected abundances of atomic species that are not supported, as well as a smaller reduction of 0.09 dex for species such as Fe which are supported by $g_{\mathrm{rad}}$. The fit for the abundances of 68 Tau is about the same for both values of $Z$ as seen in Fig. 22. Equivalently, compensating the change of $\mathrm{Fe}$ abundance would require reducing the mass loss rate from $10^{-13} M_{\odot} \mathrm{yr}^{-1}$ to $7 \times 10^{-14} M_{\odot} \mathrm{yr}^{-1}$ according to the results shown in Fig. 11. This may be viewed as the uncertainty on the mass loss rate resulting from the uncertainty of solar abundances ${ }^{22}$.

\subsection{Further implications}

Atomic diffusion alone cannot explain all characteristics of the Hyades lithium gap, nor can unseparated mass loss. The cold

\footnotetext{
22 To significantly improve the evaluation of the effect of changing to another set of solar abundances would require first recalibrating the mixing lenght using a solar model, then carrying out calculations for AmFm stars for both turbulence and mass loss, as well as reanalyzing observations of AmFm star abundances using the new solar abundances. This is outside the scope of the present paper.
} 
side of the gap can only be reproduced by diffusion only models or models with $\dot{M} \leq 1 \times 10^{-14} M_{\odot} \mathrm{yr}^{-1}$, whereas the hot side of the gap and the AmFm character of stars for $T_{\text {eff }} \geq 7200 \mathrm{~K}$ require a stronger mass loss rate. Moreover, separated mass loss (see Sects. 5.3.5 and 7.2.1) seems required to explain observed Li underabundances near the bottom of the gap as well as reduce the calculated Fe overabundances. In Fig. 18, in comparison to the diffusion only model, the curve for case 2 shows both larger underabundances of $\mathrm{Li}$ as well as smaller overabundances of Fe. Well tuned fully separated mass loss (case 1) could do the same. Similarly, the model for case 3 has a nearly flat $\mathrm{Fe}$ surface abundance coupled with similar Li underabundances to the diffusion only model. It does not seem justified to further speculate on the role of separated winds in Li gap stars until we have a better understanding of separation mecanisms within stellar winds. Since radiative forces generally increase with $T_{\text {eff }}$, the above mentioned increase in mass loss rate seems possible if winds of A and hot F stars are radiative in nature. Since a star's $T_{\text {eff }}$ changes over time, a mass loss rate which depends on $T_{\text {eff }}$ (or on $L_{*}$ ) could also vary in time (Swenson \& Faulkner 1992). Such effects were not introduced in order to limit the number of adjustable parameters.

The competition between atomic diffusion and meridional circulation in 2D should lead to solutions which resemble those obtained with mass loss, since meridional circulation leads to an additional advective term in the transport equation (Eq. (7)). Therefore, because the internal distribution of elements in the mass loss regime differs considerably from the variations encountered in the turbulent mixing regime (see discussion in Sect.6), internal distributions due to meridional circulation could also differ significantly from those encountered via turbulence. Hence, when building stellar models, one should be cautious when replacing meridional circulation, which is an advective process, by turbulent mixing. A careful study of the atomic diffusion of metals within the context of meridional circulation, such as the one carried out for helium in Quievy et al. (2009), could help determine the implications of such an approximation. This could perhaps lead to asteroseismic tests which could distinguish between models using rotationally induced turbulence (Talon et al. 2006) and those using meridional circulation (Charbonneau \& Michaud 1988), which are both used to explain the disappearance of the AmFm character for rotation velocities greater than $100 \mathrm{~km} \mathrm{~s}^{-1}$.

Observations of rapid p-mode oscillations in many Ap stars (Kurtz 1978) and in particular in Przybylski's star (see also Mkrtichian et al. 2008) have led to a number of studies of the oscillation mechanisms. In particular, Vauclair et al. (1991) suggested that unseparated mass loss acting solely in polar regions, where the magnetic field is strongest, could induce helium gradients which are compatible with oscillation generating models (Balmforth et al. 2001). Although differences between our stellar model and the one of Vauclair et al. (1991) could have an effect on the predicted anomalies (notably the absence of convection due to magnetic braking/freezing in the latter), our calculations suggest anomalies would reach inwards to about $25 \%$ of the star's radius and could have an important effect on opacities. Though it would depend on the strength of the overall mass loss rate, which will be smaller than the mass loss rate at the poles, similar He depletions could be coupled with overabundances of iron peak elements around $200000 \mathrm{~K}$ and perhaps iron convection. It is not clear whether magnetic braking/freezing or thermohaline convection can stabilize iron peak convection. Unfortunately, we are not able to investigate this scenario any further since our models require spherical symmetry.
Perhaps asteroseismology will allow us to answer some of these questions, while revealing the relative importance of meridional circulation, turbulence and mass loss within chemically peculiar stars.

Acknowledgements. We would like to thank G. Alecian as well as the anonymous referee for useful comments which allowed us to improve the paper. M. Vick thanks the Département de physique de l'Université de Montréal for financial support, as well as everyone at the GRAAL in Montpellier for their amazing hospitality. We acknowledge the financial support of Programme National de Physique Stellaire (PNPS) of CNRS/INSU, France. This research was partially supported by NSERC at the Université de Montreál. Finally, we thank the Réseau québécois de calcul de haute performance (RQCHP) for providing us with the computational resources required for this work.

\section{References}

Abbott, D. C. 1982, ApJ, 259, 282

Abt, H. A. 2000, ApJ, 544, 933

Alecian, G. 1986, A\&A, 168, 204

Alecian, G. 1996, A\&A, 310, 872

Anders, E., \& Grevesse, N. 1989, Geochim. Cosmochim. Acta, 53, 197

Anthony-Twarog, B. J., Deliyannis, C. P., Twarog, B. A., Croxall, K. V., \& Cummings, J. D. 2009, AJ, 138, 1171

Asplund, M., Grevesse, N., \& Sauval, A. J. 2005, in Cosmic Abundances as Records of Stellar Evolution and Nucleosynthesis, ed. T. G. Barnes, III \& F. N. Bash, ASP Conf. Ser., 336, 25

Asplund, M., Grevesse, N., Sauval, A. J., \& Scott, P. 2009, ARA\&A, 47, 481

Babel, J. 1995, A\&A, 301, 823

Babel, J. 1996, A\&A, 309, 867

Balachandran, S. 1995, ApJ, 446, 203

Balmforth, N. J., Cunha, M. S., Dolez, N., Gough, D. O., \& Vauclair, S. 2001, MNRAS, 323, 362

Basu, S., Chaplin, W. J., Elsworth, Y., et al. 2007, ApJ, 655, 660

Bertin, P., Lamers, H. J. G. L. M., Vidal-Madjar, A., Ferlet, R., \& Lallement, R. 1995, A\&A, 302, 899

Boesgaard, A. M., \& Budge, K. G. 1988, ApJ, 332, 410

Boesgaard, A. M., \& Friel, E. D. 1990, ApJ, 351, 467

Boesgaard, A. M., \& King, J. R. 2002, ApJ, 565, 587

Boesgaard, A. M., \& Tripicco, M. J. 1986, ApJ, 302, L49

Boesgaard, A. M., Armengaud, E., King, J. R., Deliyannis, C. P., \& Stephens, A. 2004, ApJ, 613, 1202

Borsenberger, J., Praderie, F., \& Michaud, G. 1979, A\&A, 76, 287

Brown, A., Veale, A., Judge, P., Bookbinder, J. A., \& Hubeny, I. 1990, ApJ, 361, 220

Burgers, J. S. 1969, in Flow Equations for Composite Gases (New York: Academic Press)

Burkhart, C., \& Coupry, M. F. 1989, A\&A, 220, 197

Burkhart, C., \& Coupry, M. F. 1991, A\&A, 249, 205

Burkhart, C., \& Coupry, M. F. 2000, A\&A, 354, 216

Caffau, E., Ludwig, H., Steffen, M., et al. 2008, A\&A, 488, 1031

Carrier, F., Eggenberger, P., Leyder, J., Debernardi, Y., \& Royer, F. 2007, A\&A, 470, 1009

Cayrel, R., \& Burkhart, C. C. V. 1991, ed. G. Michaud, \& A. Tutukov, IAU Symp., 145, 99

Cayrel, R., Cayrel de Strobel, G., \& Campbell, B. 1985, A\&A, 146, 249

Charbonneau, P. 1993, ApJ, 405, 720

Charbonneau, P., \& Michaud, G. 1988, ApJ, 327, 809

Delahaye, F., \& Pinsonneault, M. H. 2006, ApJ, 649, 529

Delahaye, F., Pinsonneault, M. H., Pinsonneault, L., \& Zeippen, C. J. 2010, ApJL, submitted [1005.0423]

Feldman, U., \& Widing, K. G. 2003, Space Sci. Rev., 107, 665

Feldman, W. C., Asbridge, J. R., Bame, S. J., \& Gosling, J. T. 1977, in The Solar Output and its Variation, ed. O. R. White, 351

Fossati, L., Bagnulo, S., Monier, R., et al. 2007, A\&A, 476, 911

Gatewood, G. D., \& Gatewood, C. V. 1978, ApJ, 225, 191

Gebran, M., \& Monier, R. 2008, A\&A, 483, 567

Gebran, M., Monier, R., \& Richard, O. 2008, A\&A, 479, 189

Geiss, J., \& Buergi, A. 1986, A\&A, 159, 1

Gonzalez, J.-F., LeBlanc, F., Artru, M.-C., \& Michaud, G. 1995, ApJ, 297, 223

Gratton, R. 2000, in Stellar Clusters and Associations: Convection, Rotation, and Dynamos, ed. R. Pallavicini, G. Micela, \& S. Sciortino, ASP Conf. Ser., 198, 225

Grevesse, N., Noels, A., \& Sauval, A. J. 1996, in Cosmic Abundances, ed. S. S.

Holt, \& G. Sonneborn, ASP Conf. Ser., 99, 117

Griffin, R. E. 2002, AJ, 123, 988 
A\&A 521, A62 (2010)

Hill, G. M. 1995, A\&A, 294, 536

Hill, G. M., \& Landstreet, J. D. 1993, A\&A, 276, 142

Hui-Bon-Hoa, A. 1999, A\&A, 343, 261

Hui-Bon-Hoa, A. 2000, A\&AS, 144, 203

Hui-Bon-Hoa, A., \& Alecian, G. 1998, A\&A, 332, 224

Hui-Bon-Hoa, A., Burkhart, C., \& Alecian, G. 1997, A\&A, 323, 901

Iglesias, C. A., \& Rogers, F. 1996, ApJ, 464, 943

Kato, S. 1966, PASJ, 18, 374

Korn, A. J., Grundahl, F., Richard, O., et al. 2006, Nature, 442, 657

Krishna-Swamy, K. S. 1966, ApJ, 145, 176

Krtička, J., Owocki, S. P., Kubát, J., Galloway, R. K., \& Brown, J. C. 2003, A\&A, 402, 713

Kurtz, D. W. 1978, Inform. Bull. Variable Stars, 1436, 1

Lambert, D. L., Roby, S. W., \& Bell, R. A. 1982, ApJ, 254, 663

Lamers, H. J. G. L. M., \& Cassinelli, J. P. 1999, Introduction to Stellar Winds (Introduction to Stellar Winds), ed. J. G. L. M. L. Henny, \& J. P. Cassinelli (Cambridge, UK: Cambridge University Press), 452

Landstreet, J. D., Dolez, N., \& Vauclair, S. 1998, A\&A, 333, 977

Langer, N., El Eid, M. F., \& Fricke, K. J. 1985, A\&A, 145, 179

Lanz, T., \& Catala, C. 1992, A\&A, 257, 663

LeBlanc, F., \& Alecian, G. 2008, A\&A, 477, 243

LeBlanc, F., Michaud, G., \& Richer, J. 2000, ApJ, 538, 876

Lemke, M. 1989, A\&A, 225, 125

Lemke, M. 1990, A\&A, 240, 331

Liebert, J., Young, P. A., Arnett, D., Holberg, J. B., \& Williams, K. A. 2005, ApJ, 630, L69

Maeder, A. 1997, A\&A, 321, 134

Meyer, J.P. 1985, ApJS, 57, 173

Meyer, J.-P. 1996, in Cosmic Abundances, ed. S. S. Holt, \& G. Sonneborn, ASP Conf. Ser., 99, 127

Michaud, G. 1986, ApJ, 302, 650

Michaud, G., \& Charbonneau, P. 1991, Space Sci. Rev., 57, 1

Michaud, G., \& Charland, Y. 1986, ApJ, 311, 326

Michaud, G., \& Proffitt, C. R. 1993, in Inside the Stars, ed. W. W. Weiss, \& A. Baglin, ASP Conf. Ser., 40, IAU Colloq., 137, 246

Michaud, G., Tarasick, D., Charland, Y., \& Pelletier, C. 1983, ApJ, 269, 239

Michaud, G., Richard, O., Richer, J., \& VandenBerg, D. A. 2004, ApJ, 606, 452

Michaud, G., Richer, J., \& Richard, O. 2005, ApJ, 623, 442

Michaud, G., Richer, J., \& Richard, O. 2007, ApJ, 670, 1178

Michaud, G., Richer, J., \& Richard, O. 2008, ApJ, 675, 1223

Mkrtichian, D. E., Hatzes, A. P., Saio, H., \& Shobbrook, R. R. 2008, A\&A, 490, 1109

Monier, R. 2005, A\&A, 442, 563

Neff, J. E., \& Simon, T. 2008, ApJ, 685, 478

Netopil, M., Paunzen, E., Maitzen, H. M., North, P., \& Hubrig, S. 2008, A\&A, 491,545
Owocki, S. P., \& Puls, J. 2002, ApJ, 568, 965

Palacios, A., Talon, S., Charbonnel, C., \& Forestini, M. 2003, A\&A, 399, 603

Paquette, C., Pelletier, C., Fontaine, G., \& Michaud, G. 1986, ApJS, 61, 177

Parker, E. N. 1960, ApJ, 132, 821

Perryman, M. A. C., Brown, A. G. A., Lebreton, Y., et al. 1998, A\&A, 331, 81 Preston, G. W. 1974, ARA\&A, 12, 257

Quievy, D., Charbonneau, P., Michaud, G., \& Richer, J. 2009, A\&A, 500, 1163

Randich, S., Primas, F., Pasquini, L., Sestito, P., \& Pallavicini, R. 2007, A\&A, 469, 163

Richard, O., Michaud, G., \& Richer, J. 2001, ApJ, 558, 377

Richard, O., Michaud, G., \& Richer, J. 2005, ApJ, 619, 538

Richer, J., \& Michaud, G. 1993, ApJ, 416, 312

Richer, J., \& Michaud, G. 2005, EAS Publ. Ser. 17, ed. G. Alecian, O. Richard, \& S. Vauclair, 53

Richer, J., Michaud, G., \& Massacrier, G. 1997, A\&A, 317, 968

Richer, J., Michaud, G., Rogers, F., Turcotte, S., \& Iglesias, C. A. 1998, ApJ, 492, 833

Richer, J., Michaud, G., \& Turcotte, S. 2000, ApJ, 529, 338

Roby, S. W., \& Lambert, D. L. 1990, ApJS, 73, 67

Simon, T., \& Landsman, W. B. 1997, ApJ, 483, 435

Springmann, U. W. E., \& Pauldrach, A. W. A. 1992, A\&A, 262, 515

Swenson, F. J., \& Faulkner, J. 1992, ApJ, 395, 654

Takeda, Y., \& Sadakane, K. 1997, PASJ, 49, 367

Talon, S., \& Charbonnel, C. 2003, A\&A, 405, 1025

Talon, S., \& Charbonnel, C. 2005, A\&A, 440, 981

Talon, S., Richard, O., \& Michaud, G. 2006, ApJ, 645, 634

Théado, S., Vauclair, S., Alecian, G., \& Le Blanc, F. 2009, ApJ, 704, 1262

Turcotte, S., Michaud, G., \& Richer, J. 1998a, ApJ, 504, 559

Turcotte, S., Richer, J., Michaud, G., Iglesias, C. A., \& Rogers, F. 1998b, ApJ, 504, 539

Turcotte, S., Richer, J., Michaud, G., \& Christensen-Dalsgaard, J. 2000, A\&A, 360,603

Unglaub, K. 2008, A\&A, 486, 923

VandenBerg, D. A., Edvardsson, B., Eriksson, K., \& Gustafsson, B. 2008, ApJ, 675,746

Varenne, O., \& Monier, R. 1999, A\&A, 351, 247

Vauclair, S. 1975, A\&A, 45, 233

Vauclair, S. 1988, ApJ, 335, 971

Vauclair, S., \& Charbonnel, C. 1995, A\&A, 295, 715

Vauclair, S., Dolez, N., \& Gough, D. O. 1991, A\&A, 252, 618

Watson, W. D. 1971, A\&A, 13, 263

Wiese, W. L., Smith, M. W., \& Glennon, B. M. 1966, Atomic transition probabilities, Hydrogen through Neon. A critical data compilation, NSRDSNBS 4, Washington, D.C.: US Department of Commerce, National Buereau of Standards 